\title{
A Large Term Rewrite System Modelling a Pioneering Cryptographic Algorithm
}

\author{
Hubert Garavel Lina Marsso \\ INRIA Grenoble, France \\ Univ. Grenoble Alpes, LIG, F-38000 Grenoble, France \\ CNRS, LIG, F-38000 Grenoble, France \\ Hubert.Garavel@inria.fr Lina.Marsso@inria.fr
}

\begin{abstract}
We present a term rewrite system that formally models the Message Authenticator Algorithm (MAA), which was one of the first cryptographic functions for computing a Message Authentication Code and was adopted, between 1987 and 2001, in international standards (ISO 8730 and ISO 8731-2) to ensure the authenticity and integrity of banking transactions. Our term rewrite system is large (13 sorts, 18 constructors, 644 non-constructors, and 684 rewrite rules), confluent, and terminating. Implementations in thirteen different languages have been automatically derived from this model and used to validate 200 official test vectors for the MAA.
\end{abstract}

\section{Introduction}

In data security, a Message Authentication Code (MAC) is a short sequence of bits that is computed from a given message; the MAC ensures both the authenticity and integrity of the message, i.e., that the message sender is the stated one and that the message contents have not been altered. A MAC is more than a mere checksum, as it must be secure enough to defeat attacks; its design usually involves cryptographic keys shared between the message sender and receiver.

One of the first MAC algorithm to gain widespread acceptance was the Message Authenticator Algorithm (also known as Message Authentication Algorithm, MAA for short) [1] [2] [18] designed in 1983 by Donald Davies and David Clayden at the National Physical Laboratory (NPL) in response to a request of the UK Bankers Automated Clearing Services. The MAA was adopted by ISO in 1987 and became part of the international standards 8730 [10] and 8731-2 [11]. Later, cryptanalysis of MAA revealed various weaknesses, including feasible brute-force attacks, existence of collision clusters, and key-recovery techniques [19] [22] [21] [20] [18]. For this reason, MAA was withdrawn from ISO standards in 2002.

From the point of view of formal methods, the MAA is interesting because of its pioneering nature, because its definition is freely available and stable, and because it is involved enough while remaining of manageable complexity. Over the past decades, various formal specifications of the MAA have been developed using VDM, Z, abstract data types (i.e., algebraic specifications), term rewrite systems, etc. For such formalisms, the usual examples often deal with syntax trees, which are explored using standard traversals (breadth-first, depth-first, etc.); contrary to such commonplace examples, cryptographic functions (and the MAA, in particular) exhibit more diverse behaviour, as they rather seek to perform irregular computations than linear ones.

The present article is organized as follows. Section 2 provides an algorithmic overview of the MAA. Section 3 lists the preexisting formal specifications of the MAA. Section 4 presents the modelling of the MAA using term rewrite systems. Section 5 discusses validation steps applied to this model. Finally, Section 6 gives concluding remarks. 


\section{Overview of the MAA}

Nowadays, Message Authentication Codes are computed using different families of algorithms based on either cryptographic hash functions (HMAC), universal hash functions (UMAC), or block ciphers (CMAC, OMAC, PMAC, etc.). Contrary to these modern approaches, the MAA was designed as a standalone algorithm that does not rely on any preexisting hash function or cipher.

In this section, we briefly explain the principles of the MAA. More detailed explanations can be found in [1], [2] and [14, Algorithm 9.68].

The MAA was intended to be implemented in software and to run on 32-bit computers. Hence, its design intensively relies on 32-bit words (called blocks) and 32-bit machine operations.

The MAA takes as inputs a key and a message. The key has 64 bits and is split into two blocks $J$ and $K$. The message is seen as a sequence of blocks. If the number of bytes of the message is not a multiple of four, extra null bytes are added at the end of the message to complete the last block. The size of the message should be less than 1,000,000 blocks; otherwise, the MAA result is said to be undefined; we believe that this restriction, which is not inherent to the algorithm itself, was added in the ISO 8731-2 standard to provide MAA implementations with an upper bound (four megabytes) on the size of memory buffers used to store messages.

The MAA produces as output a block, which is the MAC value computed from the key and the message. The fact that this result has only 32 bits proved to be a major weakness enabling cryptographic attacks; MAC values computed by modern algorithms now have a much larger number of bits. Apart from the aforementioned restriction on the size of messages, the MAA behaves as a totally-defined function; its result is deterministic in the sense that, given a key and a message, there is only a single MAC result, which neither depends on implementation choices nor on hidden inputs, such as nonces or randomly-generated numbers.

The MAA calculations rely upon conventional 32-bit logical and arithmetic operations, among which: AND (conjunction), OR (disjunction), XOR (exclusive disjunction), CYC (circular rotation by one bit to the left), ADD (addition), CAR (carry bit generated by 32-bit addition), MUL (multiplication, sometimes decomposed into HIGH_MUL and LOW_MUL, which denote the most- and least-significant blocks in the 64bit product of a 32-bit multiplication). On this basis, more involved operations are defined, among which MUL1 (result of a 32-bit multiplication modulo $2^{32}-1$ ), MUL2 (result of a 32-bit multiplication modulo $2^{32}-2$ ), MUL2A (faster version of MUL2), FIX1 and FIX2 (two unary functions ${ }^{1}$ respectively defined as $x \rightarrow \operatorname{AND}(\mathrm{OR}(x, \mathrm{~A}), \mathrm{C})$ and $x \rightarrow \operatorname{AND}(\mathrm{OR}(x, \mathrm{~B}), \mathrm{D})$, where $\mathrm{A}, \mathrm{B}, \mathrm{C}$, and $\mathrm{D}$ are four hexadecimal block constants $\mathrm{A}=02040801, \mathrm{~B}=00804021, \mathrm{C}=\mathrm{BFEF7FDF}$, and $\mathrm{D}=7 \mathrm{DFEFBFF})$. The MAA operates in three successive phases:

- The prelude takes the two blocks $J$ and $K$ of the key and converts them into six blocks $X_{0}, Y_{0}, V_{0}$, $W, S$, and $T$. This phase is executed once. After the prelude, $J$ and $K$ are no longer used.

- The main loop successively iterates on each block of the message. This phase maintains three variables $X, Y$, and $V$ (initialized to $X_{0}, Y_{0}$, and $V_{0}$, respectively), which are modified at each iteration. The main loop also uses the value of $W$, but neither $S$ nor $T$.

- The coda adds the blocks $S$ and $T$ at the end of the message and performs two more iterations on these blocks. After the last iteration, the MAA result is $\mathrm{XOR}(X, Y)$.

In 1987, the ISO 8731-2 standard [9, Section 5] introduced an additional feature (called mode of operation), which concerns messages longer than 256 blocks and which, seemingly, was not present

${ }^{1}$ The names FIX1 and FIX2 are borrowed from [15, pages 36 and 77]. 
in the early MAA versions designed at NPL. Each message longer than 256 blocks must be split into segments of 256 blocks each, with the last segment possibly containing less than 256 blocks. The above MAA algorithm (prelude, main loop, and coda) is applied to the first segment, resulting in a value noted $Z_{1}$. This block $Z_{1}$ is then inserted before the first block of the second segment, leading to a 257-block message to which the MAA algorithm is applied, resulting in a value noted $Z_{2}$. This is done repeatedly for all the $n$ segments, the MAA result $Z_{i}$ computed for the $i$-th segment being inserted before the first block of the $(i+1)$-th segment. Finally, the MAC for the entire message is the MAA result $Z_{n}$ computed for the last segment.

\section{Prior Formal Specifications of the MAA}

The informal description of the MAA can be found both in ISO standard 8731-2 [11] or in a 1988 NPL technical report [2]. On this basis, several formal models of the MAA have been developed:

- In 1990, G. I. Parkin and G. O’Neill designed a formal specification of the MAA in VDM [16] [17]. To our knowledge, this was the first attempt at applying formal methods to the MAA. The VDM specification became part of the ISO standard defining the MAA [11, Annex B]. Three implementations in C [16, Annex C], Miranda [16, Annex B], and Modula-2 [13] were written by hand along the lines of the VDM specification.

- In 1991, M. K. F. Lai formally described the MAA using the set-theoretic Z notation [12]. He adopted Knuth's "literate programming" approach, by inserting formal fragments of $Z$ code in the natural-language description of the MAA.

- In 1991, Harold B. Munster produced a formal specification of the MAA in LOTOS [15]. The MAA was described using only the data part of LOTOS: the behavioural part of LOTOS, which serves to describe concurrent processes, was not used. The LOTOS specification, which made intensive use of the predefined LOTOS data-type libraries, was mainly declarative but not executable, as all facilities of LOTOS abstract data types were used in an unconstrained way. For instance, some equations could be rephrased as: "given $x$, the result is y such that $x=f(y)$ ", which required to invert function $f$ in order to compute $y$.

- In 1992, Hubert Garavel and Philippe Turlier, taking the aforementioned LOTOS specification as a starting point, gradually transformed it by successive modifications. Their goal was to obtain an executable specification that could be processed by the CÆSAR.ADT compiler [6] [7], while staying as close as possible to the original LOTOS specification. To do so, three main kinds of modifications were applied: (i) the LOTOS algebraic equations, which are not oriented, were turned into rewrite rules, which are oriented from left to right and, thus, more amenable to automatic execution; (ii) a distinction was made between constructor and non-constructor operations, and the discipline of "free" constructors was enforced - namely, each rule defining a non-constructor $f$ must have the form " $f\left(P_{1}, \ldots, P_{n}\right) \rightarrow \ldots$.", where each $P_{i}$ contains only constructors and free variables; (iii) some LOTOS sorts and operations were implemented as C types and functions, by importing manually-written $\mathrm{C}$ code - for instance, addition, multiplication, and bit shifts on 32-bit words were implemented directly in $\mathrm{C}$. From this specification, the CÆSAR.ADT compiler could automatically generate $\mathrm{C}$ code that, combined with a small handwritten main program, computed the MAC value corresponding to a message and a key. 


\section{Specification of the MAA as a Term Rewrite System}

Taking Garavel \& Turlier's LOTOS specification as a starting point, our central contribution is a formal model of the MAA specified as a term rewrite system. This model is expressed using the notations of the simple rewriting language REC proposed in [5, Sect. 3] and [4, Sect. 3.1], which was lightly enhanced to distinguish between free constructors (declared in the "CONS" part) and non-constructors (declared in the "OPNS" part and defined by rewrite rules given in the "RULES" part).

The model is given in Annex B of the present article. Notice that the model has a few (only six) conditional rules and is thus a conditional term rewrite system; if needed, it could easily be turned into a non-conditional term rewrite system by slightly modifying the definitions of three functions (i.e., adding extra parameters and auxiliary functions), as explained in Annex B. Our main results are the following:

- Our model is large. It is 1575-line long and contains 13 sorts, 18 constructors, 644 nonconstructors, and 684 rewrite rules. Although research on term rewriting led to a wealth of scientific publications, it is difficult to find concrete examples of large term-rewrite systems: for instance, in the data base of models accumulated during the three Rewrite Engines Competition (2006, 2008, and 2010), the largest models are less than 300-line long. There exist indeed larger (e.g., 10,000-line long) term rewrite systems, but they are either generated automatically (and, thus, difficult to understand by humans) or they are actual implementations of compilers or translators (and, often, are not "pure" term rewrite systems, as they rely upon higher-level features, e.g., subsorts or strategies).

- Our model is exhaustive, as it fully describes the MAA algorithm, including its "mode of operation" and its segmentation of messages larger than 1024 bytes.

- Our model is self-contained, as each detail of the MAA is expressed using term rewrite systems only; the model does not rely upon any externally-defined type or function and is thus independent from machine-specific assumptions, e.g., 32-bit vs 64-bit words or little- vs big-endian ordering.

- Our model is executable. From a theoretical point of view, this was enabled by the aforementioned shift from general LOTOS abstract data types to term rewrite systems, which are less declarative and more operational. From a practical point of view, this shift was not sufficient, as the MAA intensively manipulates block values (i.e., 32-bit numbers), which cannot be reasonably implemented in the Peano style (the execution stack quickly overflows when these numbers are represented using the zero and succ constructors). To overcome this issue, we chose to represent blocks in binary form, as words of four octets. So doing, the logical operations on blocks (AND, $\mathrm{OR}, \mathrm{XOR}$, and CYC) are easy to define using bitwise and octetwise manipulations. The arithmetical operations (ADD, CAR, and MUL) are more involved: we implemented them using 8-bit, 16-bit, and 32-bit adders and multipliers, more or less inspired from the theory of digital circuits.

- Our model is minimal, in the sense that each sort, constructor, and non-constructor defined in our model is actually used (i.e., the model does not contain "dead" code).

- Our model is readable. Despite its size, efforts have been made to give it a modular structure, which is reflected in the sections of Annex B. Particular care has been taken to choose constructors appropriately and to keep non-constructor definitions as simple as possible. 


\section{Validation of the MAA Model}

In this section, we detail the various steps performed to make sure that our model is correct:

- Our model is self-checking. Because the REC language has no input/output primitive and no provision for interfacing with external $\mathrm{C}$ code, it cannot be used to compute the MAC value of a given file. In order to check whether our model was correct or not, we enriched it with 203 test vectors originating from three sources, namely: (i) all the test vectors provided in Tables 1 to 6 of [11, Annex A] and [2]; (ii) all the test vectors provided in [10, Annex E.3.3] - the subsequent test vectors of [10, Annexes E.3.4 and E.4] were discarded because of their size (they deal with two messages having 84 and 588 blocks, which would have led to a much too large REC specification); (iii) supplementary test vectors intended to specifically check for certain aspects (byte permutations and message segmentation) that were not enough covered by the above tests; this was done by introducing a makeMessage function acting as a pseudo-random message generator (see Annex B.12).

- Our model is confluent. This is easy to see, because all constructors are free and all the rules defining non-constructors have disjoint patterns and mutually exclusive premises; for safety, the disjunction of patterns and exclusion of guards has been checked mechanically by translating the REC specification to the Opal language [3], whose compiler emits warnings in presence of "ambiguous" (i.e., nondeterministic) rules.

- Our model is terminating. This has been verified by automatically translating our REC model into the input formalism TRS of the AProVE tool [8], which produced a proof of quasi-decreasingness in 76 steps.

- Our model was validated. We checked that the REC specification satisfies all the aforementioned test vectors. Because it enjoys the confluence and termination properties, all rewrite strategies should lead to the same result. Using a software framework ${ }^{2}$ under development at INRIA Grenoble, we automatically translated our REC model into thirteen different languages: Clean, Haskell, LNT, LOTOS, Maude, mCRL2, OCaml, Opal, Rascal, Scala, SML, Stratego/XT, and Tom. We submitted these translations to sixteen compilers, interpreters, and rewrite engines: eleven of them reported that all the 203 tests passed successfully, while the other tools halted or timed out. Moreover, some involved components (namely, the binary adders and multipliers) have been validated separately using more than 30,000 test vectors.

\section{Conclusion}

Twenty-five years after, we revisited the Message Authenticator Algorithm (MAA), which used to be a pioneering case study for cryptography in the 80s and for formal methods in the early $90 \mathrm{~s}$.

We developed a formal specification of the MAA, expressed as a term rewrite system encoded in the REC language. As far as we are aware, it is one of the largest handwritten term rewrite systems publicly available. This specification is self-contained and self-checking, as it includes 203 test vectors. It has been carefully validated using a dozen tools.

Parts of this specification (in particular, the binary adders and multipliers) are certainly reusable for different purposes, e.g., formal libraries for modular arithmetic or cryptography.

\footnotetext{
${ }^{2}$ http://gforge.inria.fr/scm/viewvc.php/rec/2015-CONVECS
} 
This study enabled us to discover various mistakes in prior MAA specifications. For instance, we corrected the test vectors given for function PAT at the bottom of Table 3 in [11, Annex A] and [2] (see Annex A of the present article for details). We also corrected the handwritten implementation in $\mathrm{C}$ of the function HIGH_MUL imported by the aforementioned LOTOS specification (this illustrates the risks arising when formal and non-formal codes are mixed).

It is however fair to warn the reader that term rewrite systems are a low-level theoretical model that does not scale well to large problems. The REC specification is between two and six times longer than any other (formal or informal) description of the MAA, and it took considerable effort to come up with a REC specification that is readable, properly structured, and seemingly straightforward. Similar results might not be easy to reproduce on a regular basis with other case studies.

\section{Acknowledgements}

We are grateful to Keith Lockstone for his advices and his web site ${ }^{3}$ giving useful information about the MAA, and to Sharon Wilson, librarian of the National Physical Laboratory, who provided us with valuable early NPL reports that cannot be fetched from the web.

\section{References}

[1] Donald W. Davies (1985): A Message Authenticator Algorithm Suitable for A Mainframe Computer. In G. R. Blakley \& David Chaum, editors: Advances in Cryptology - Proceedings of the Workshop on the Theory and Application of Cryptographic Techniques (CRYPTO'84), Santa Barbara, CA, USA, Lecture Notes in Computer Science 196, Springer, pp. 393-400, doi:10.1007/3-540-39568-7_30.

[2] Donald W. Davies \& David O. Clayden (1988): The Message Authenticator Algorithm (MAA) and its Implementation. NPL Report DITC 109/88, National Physical Laboratory, Teddington, Middlesex, UK. Available at http://www.cix.co.uk/ klockstone/maa.pdf.

[3] Klaus Didrich, Andreas Fett, Carola Gerke, Wolfgang Grieskamp \& Peter Pepper (1994): OPAL: Design and Implementation of an Algebraic Programming Language. In Jürg Gutknecht, editor: Proceedings of the International Conference on Programming Languages and System Architectures, Zurich, Switzerland, Lecture Notes in Computer Science 782, Springer, pp. 228-244, doi:10.1007/3-540-57840-4_34.

[4] Francisco Durán, Manuel Roldán, Jean-Christophe Bach, Emilie Balland, Mark van den Brand, James R. Cordy, Steven Eker, Luc Engelen, Maartje de Jonge, Karl Trygve Kalleberg, Lennart C. L. Kats, PierreEtienne Moreau \& Eelco Visser (2010): The Third Rewrite Engines Competition. In Peter Csaba Ölveczky, editor: Proceedings of the 8th International Workshop on Rewriting Logic and Its Applications (WRLA'10), Paphos, Cyprus, Lecture Notes in Computer Science 6381, Springer, pp. 243-261, doi:10. 1007/978-3-642-16310-4_16.

[5] Francisco Durán, Manuel Roldán, Emilie Balland, Mark van den Brand, Steven Eker, Karl Trygve Kalleberg, Lennart C. L. Kats, Pierre-Etienne Moreau, Ruslan Schevchenko \& Eelco Visser (2009): The Second Rewrite Engines Competition. Electronic Notes in Theoretical Computer Science 238(3), pp. 281-291, doi:10.1016/ j.entcs.2009.05.025.

[6] Hubert Garavel (1989): Compilation of LOTOS Abstract Data Types. In Son T. Vuong, editor: Proceedings of the 2nd International Conference on Formal Description Techniques FORTE'89 (Vancouver B.C., Canada), North-Holland, pp. 147-162. Available at http://cadp.inria.fr/publications/Garavel-89-c . html.

[7] Hubert Garavel \& Philippe Turlier (1993): CAESAR.ADT: un compilateur pour les types abstraits algébriques du langage LOTOS. In Rachida Dssouli \& Gregor v. Bochmann, editors: Actes du Colloque Francophone

\footnotetext{
${ }^{3}$ http://www.cix.co.uk/ klockstone
} 
pour l'Ingénierie des Protocoles (CFIP'93), Montréal, Canada. Available at http://cadp.inria.fr/ publications/Garavel-Turlier-93.html.

[8] Jürgen Giesl, Marc Brockschmidt, Fabian Emmes, Florian Frohn, Carsten Fuhs, Carsten Otto, Martin Plücker, Peter Schneider-Kamp, Thomas Ströder, Stephanie Swiderski \& René Thiemann (2014): Proving Termination of Programs Automatically with AProVE. In Stéphane Demri, Deepak Kapur \& Christoph Weidenbach, editors: Proceedings of the 7th International Joint Conference on Automated Reasoning (IJCAR'14), Vienna, Austria, Lecture Notes in Computer Science 8562, Springer, pp. 184191, doi:10.1007/978-3-319-08587-6_13. Available at http://verify.rwth-aachen.de/giesl/ papers/IJCAR14-AProVE.pdf.

[9] ISO (1987): Approved Algorithms for Message Authentication - Part 2: Message Authenticator Algorithm (MAA). International Standard 8731-2, International Organization for Standardization - Banking, Geneva.

[10] ISO (1990): Requirements for Message Authentication (Wholesale). International Standard 8730, International Organization for Standardization - Banking, Geneva.

[11] ISO (1992): Approved Algorithms for Message Authentication - Part 2: Message Authenticator Algorithm. International Standard 8731-2, International Organization for Standardization - Banking, Geneva.

[12] M. K. F. Lai (1991): A Formal Interpretation of the MAA Standard in Z. NPL Report DITC 184/91, National Physical Laboratory, Teddington, Middlesex, UK.

[13] R. P. Lampard (1991): An Implementation of MAA from a VDM Specification. NPL Technical Memorandum DITC 50/91, National Physical Laboratory, Teddington, Middlesex, UK.

[14] Alfred Menezes, Paul C. van Oorschot \& Scott A. Vanstone (1996): Handbook of Applied Cryptography. CRC Press, doi:10.1201/9781439821916. Available at http://cacr .uwaterloo.ca/hac.

[15] Harold B. Munster (1991): LOTOS Specification of the MAA Standard, with an Evaluation of LOTOS. NPL Report DITC 191/91, National Physical Laboratory, Teddington, Middlesex, UK. Available at ftp://ftp. inrialpes.fr/pub/vasy/publications/others/Munster-91-a.pdf.

[16] Graeme I. Parkin \& G. O'Neill (1990): Specification of the MAA Standard in VDM. NPL Report DITC 160/90, National Physical Laboratory, Teddington, Middlesex, UK.

[17] Graeme I. Parkin \& G. O'Neill (1991): Specification of the MAA Standard in VDM. In Søren Prehn \& W. J. Toetenel, editors: Formal Software Development - Proceedings (Volume 1) of the 4th International Symposium of VDM Europe (VDM'91), Noordwijkerhout, The Netherlands, Lecture Notes in Computer Science 551, Springer, pp. 526-544, doi:10.1007/3-540-54834-3_31.

[18] Bart Preneel (2011): MAA. In Henk C. A. van Tilborg \& Sushil Jajodia, editors: Encyclopedia of Cryptography and Security (2nd Edition), Springer, pp. 741-742, doi:10.1007/978-1-4419-5906-5_591.

[19] Bart Preneel \& Paul C. van Oorschot (1996): On the Security of Two MAC Algorithms. In Ueli M. Maurer, editor: Advances in Cryptology - Proceedings of the International Conference on the Theory and Application of Cryptographic Techniques (EUROCRYPT'96), Saragossa, Spain, Lecture Notes in Computer Science 1070, Springer, pp. 19-32, doi:10.1007/3-540-68339-9_3.

[20] Bart Preneel \& Paul C. van Oorschot (1999): On the Security of Iterated Message Authentication Codes. IEEE Transactions on Information Theory 45(1), pp. 188-199, doi:10.1109/18.746787.

[21] Bart Preneel, Vincent Rumen \& Paul C. van Oorschot (1997): Security Analysis of the Message Authenticator Algorithm (MAA). European Transactions on Telecommunications 8(5), pp. 455-470, doi:10.1002/ett. 4460080504.

[22] Vincent Rijmen, Bart Preneel \& Erik De Win (1996): Key Recovery and Collision Clusters for MAA. In: Proceedings of the 1st International Conference on Security in Communication Networks (SCN'96). Available at https://www.cosic.esat.kuleuven.be/publications/article-437.pdf. 


\section{A Errata Concerning Annex A of the ISO-8731-2:1992 Standard}

After checking carefully all the test vectors contained in the original NPL report defining the MAA [2] and in the 1992 version of the MAA standard [11], we believe that there are mistakes ${ }^{4}$ in the test vectors given for function PAT.

More precisely, the three last lines of Table 3 [2, page 15] — identically reproduced in Table A.3 of [11, Sect. A.4] - are written as follows:

\begin{tabular}{|c|c|c|c|c|c|}
\hline$\{\mathrm{X} 0, \mathrm{YO}\}$ & 0103 & 0703 & 1D3B & 7760 & $\operatorname{PAT}\{\mathrm{XO}, \mathrm{YO}\}$ \\
\hline$\{\mathrm{VO}, \mathrm{W}\}$ & 0103 & $050 B$ & 1706 & $5 \mathrm{DBB}$ & $\operatorname{PAT}\{\mathrm{VO}, \mathrm{W}\}$ \\
\hline$\{\mathrm{S}, \mathrm{T}\}$ & 0103 & 0705 & 8039 & 7302 & $\operatorname{PAT}\{\mathrm{S}, \mathrm{T}\}$ \\
\hline
\end{tabular}

Actually, the inputs of function PAT should not be $\{\mathrm{XO}, \mathrm{YO}\},\{\mathrm{VO}, \mathrm{W}\},\{\mathrm{S}, \mathrm{T}\}$ but rather $\{\mathrm{H} 4, \mathrm{H} 5\}$, $\{\mathrm{H} 6, \mathrm{H} 7\},\{\mathrm{H} 8, \mathrm{H} 9\}$, the values of $\mathrm{H} 4, \ldots, \mathrm{H} 9$ being those listed above in Table 3 . Notice that the confusion was probably caused by the following algebraic identities:

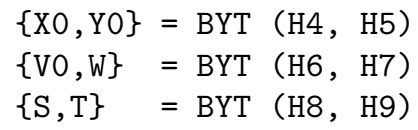

If one gives $\{\mathrm{XO}, \mathrm{YO}\},\{\mathrm{V} 0, \mathrm{~W}\},\{\mathrm{S}, \mathrm{T}\}$ as inputs to $\mathrm{PAT}$, then the three results of PAT are equal to 00 and thus cannot be equal to $\mathrm{EE}, \mathrm{BB}, \mathrm{E} 6$, respectively.

But if one gives $\{\mathrm{H} 4, \mathrm{H} 5\},\{\mathrm{H} 6, \mathrm{H} 7\},\{\mathrm{H} 8, \mathrm{H} 9\}$ as inputs to PAT, then the results of PAT are the expected values EE, BB, E6.

Thus, we believe that the three last lines of Table 3 should be modified as follows:

$\begin{array}{lllll}\{\mathrm{H} 4, \mathrm{H} 5\} & 0000000300000060 & \operatorname{PAT}\{\mathrm{H} 4, \mathrm{H} 5\} & \mathrm{EE} \\ \{\mathrm{H} 6, \mathrm{H} 7\} & 0003000000060000 & \operatorname{PAT}\{\mathrm{H} 6, \mathrm{H} 7\} & \mathrm{BB} \\ \{\mathrm{H} 8, \mathrm{H} 9\} & 0000000580000002 & \operatorname{PAT}\{\mathrm{H} 8, \mathrm{H} 9\} & \mathrm{E} 6\end{array}$

\section{B Formal Specification of the MAA in the REC Language}

This annex presents the specification of the MAA in the REC language. This specification is fully self-contained, meaning that it does not depend on any externally-defined library — with the minor disadvantage of somewhat lengthy definitions for octet and blocks constants.

For readability, the specification has been split into 21 parts, each part being devoted to a particular sort, a group of functions sharing a common purpose, or a collection of test vectors. The first parts contain general definitions that are largely independent from the MAA; starting from Sect. B.11, the definitions become increasingly MAA-specific.

The complete REC specification is obtained by concatenating all these parts, grouping their various sections (i.e., merging all SORTS sections into a single one, all CONS sections into a single one, etc.) and, after this, removing duplicated variable declarations in the VARS section, and pasting broken lines so that each rewrite rule that was split across several lines now fits on one single line.

For readability, the rewrite rules concerning the same non-constructors have been put together and separated with blank lines when appropriate. Also, the arguments of certain non-constructors are separated by semicolons rather than commas when it helps to distinguish arguments of different nature (e.g., summand bits, carry, or sum bits).

\footnotetext{
${ }^{4}$ We used the French version of this standard, but we believe that the language plays no role, as the same mistakes were already present in the 1988 NPL report.
} 
All machine words (octets, blocks, etc.) are represented according to the "big endian" convention, i.e., the first argument of each corresponding constructor denote the most significant bit.

\section{B.1 Definitions for sort Bool}

We first define Booleans using the false and true constructors, together with two non-constructors implementing logical conjunction and disjunction.

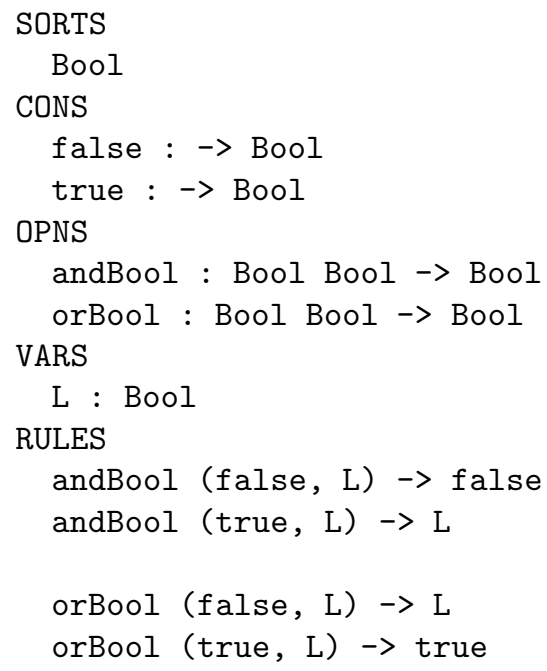

\section{B.2 Definitions for sort Nat}

We then define natural numbers in the Peano style using the zero and succ constructors, together with non-constructors implementing addition, multiplication, equality, strict order, and a few constants.

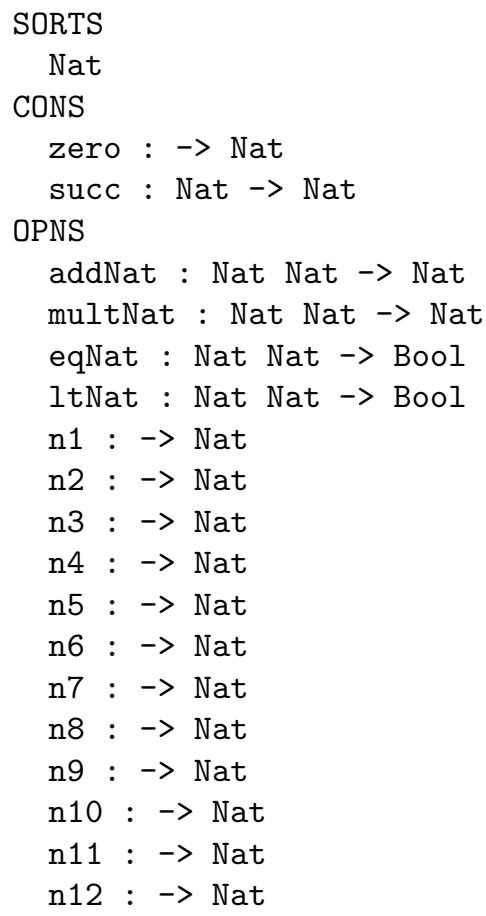




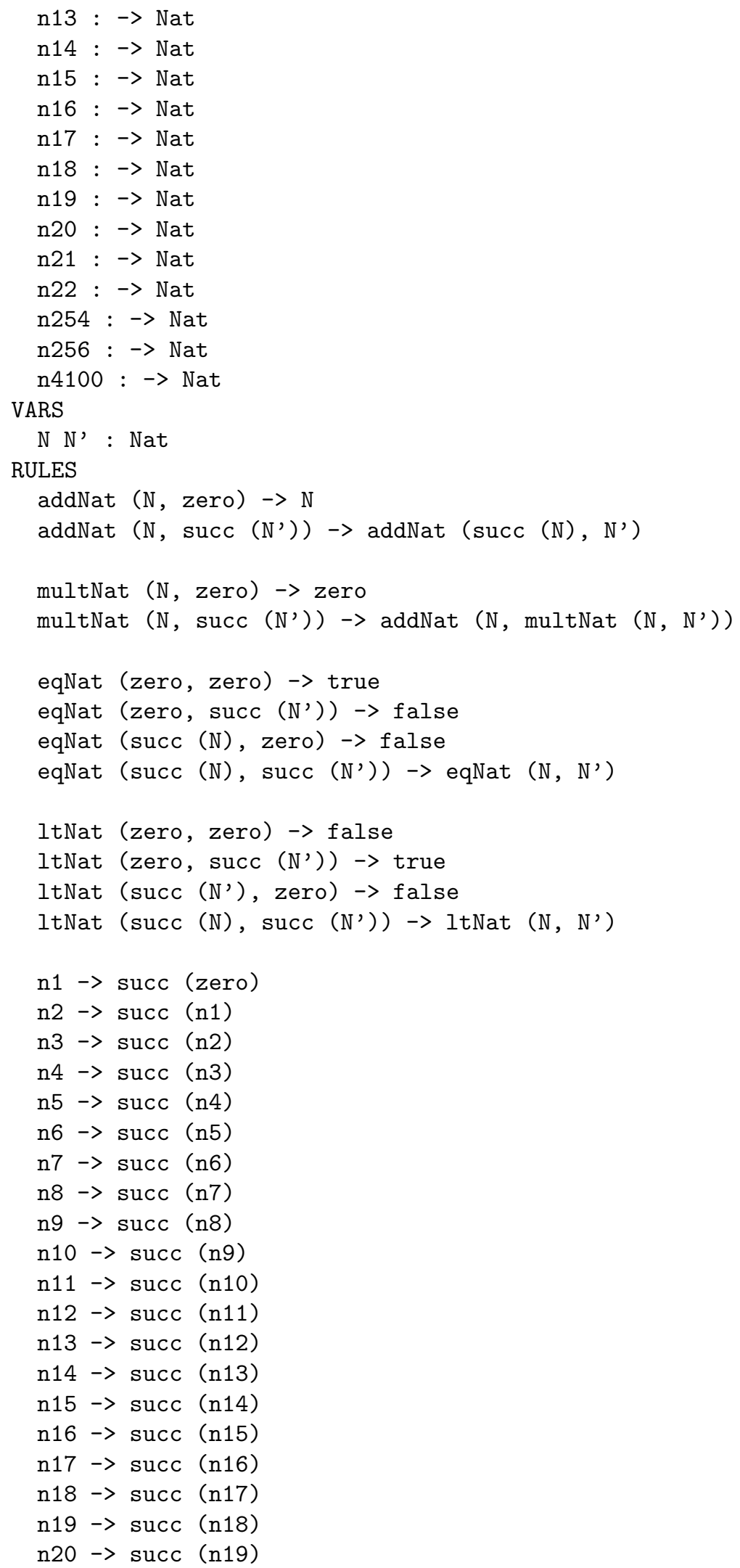




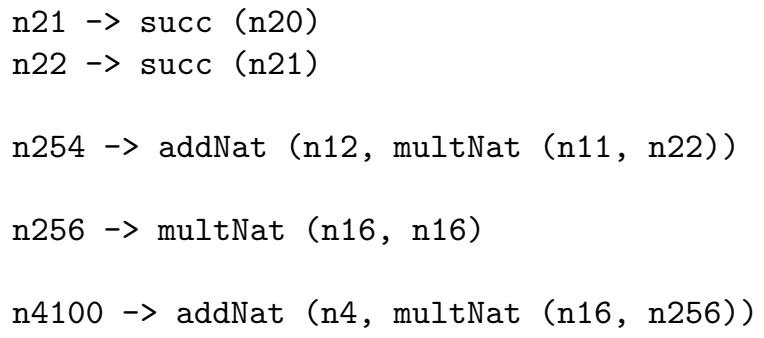

\section{B.3 Definitions for sort Bit}

We now define bits using two constructors $\mathrm{x} 0$ and $\mathrm{x} 1$, together with non-constructors implementing bit equality and logical operations on bits.

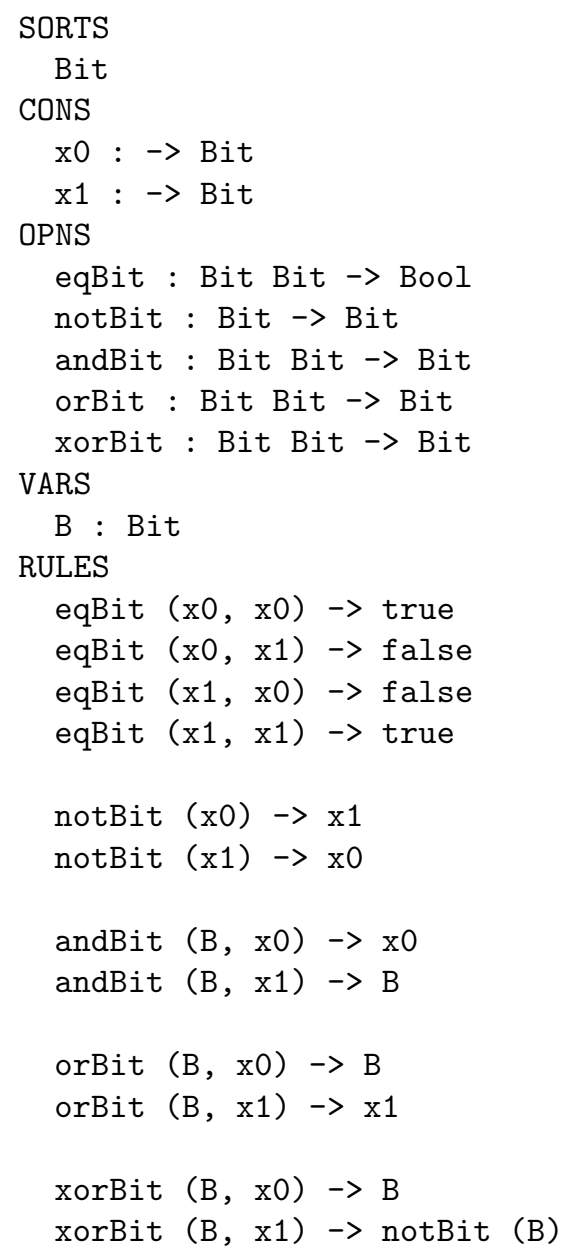

\section{B.4 Definitions for sort Octet}

We now define octets using a constructor buildOctet that takes eight bits and returns a byte, together with non-constructors implementing equality, bitwise logical operations, left-shift and right-shift operations on octets, as well as all octet constants needed to formally describe the MAA and its test vectors. 


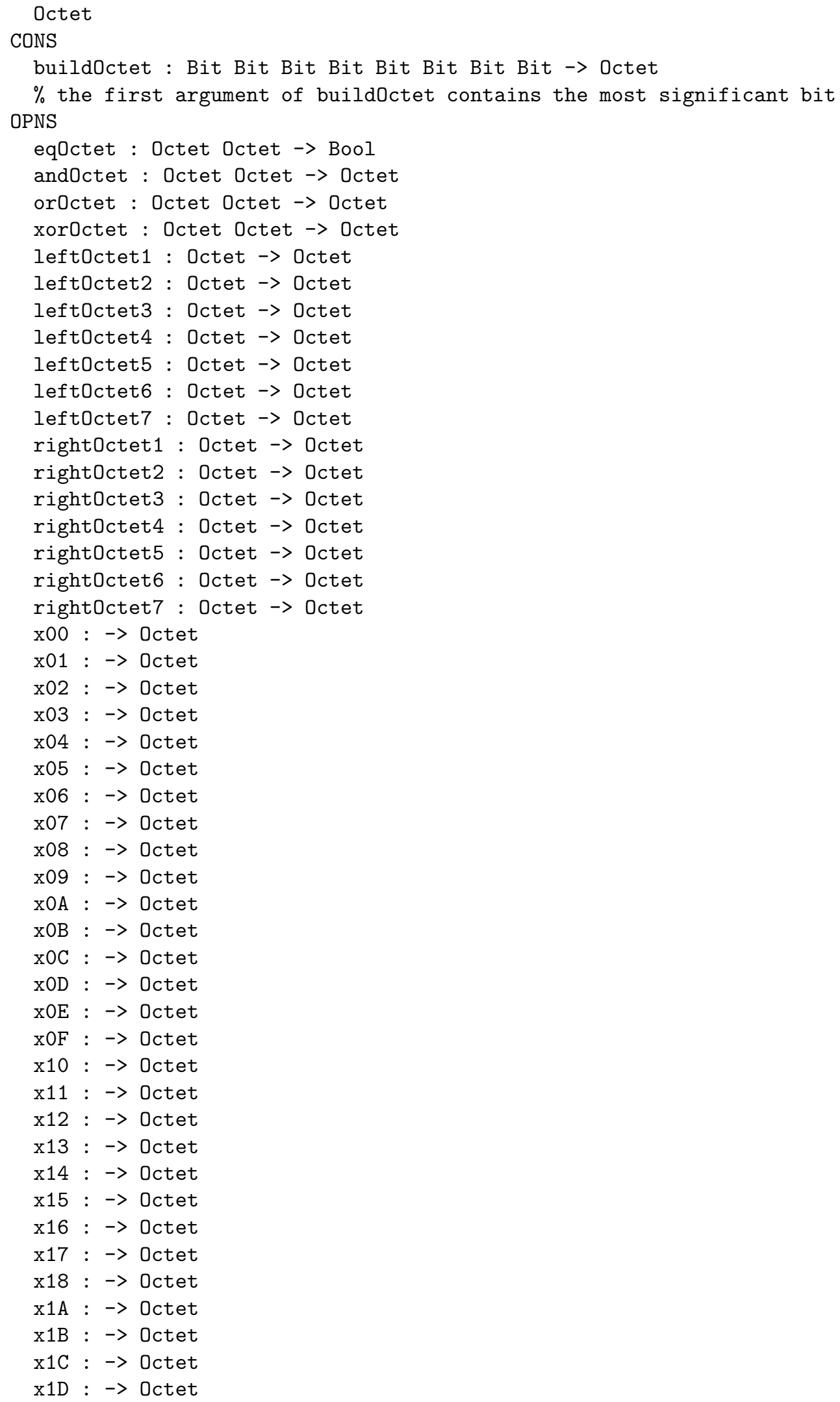




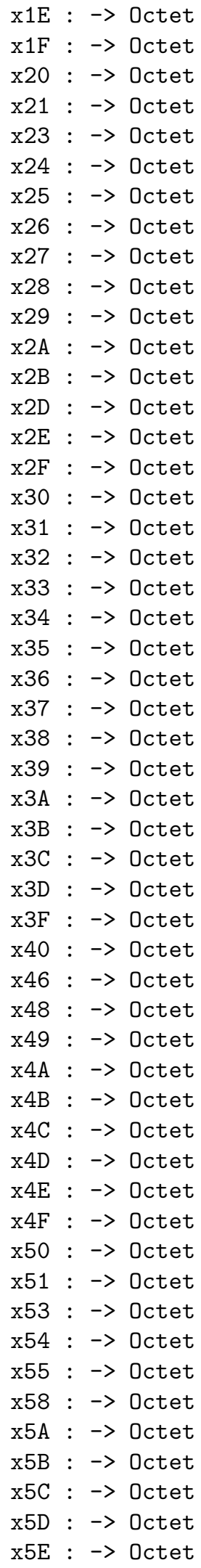




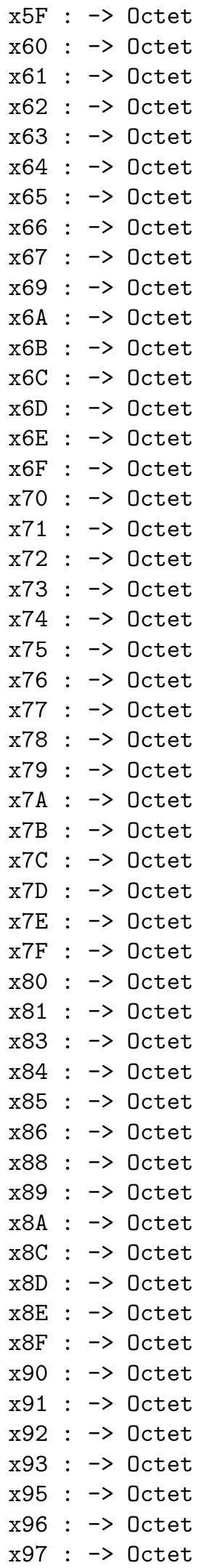




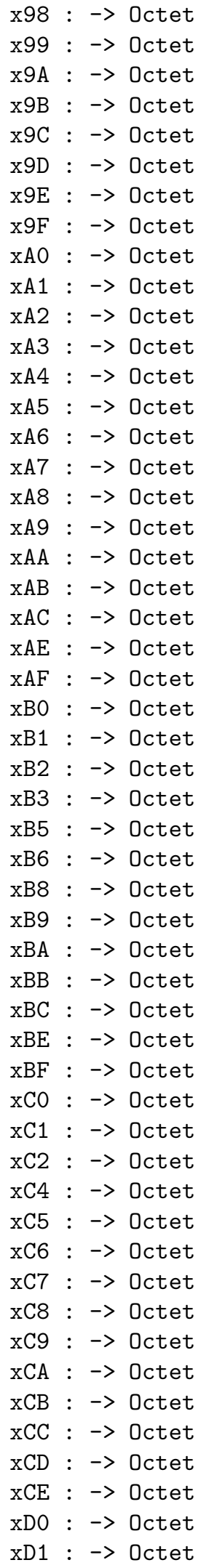




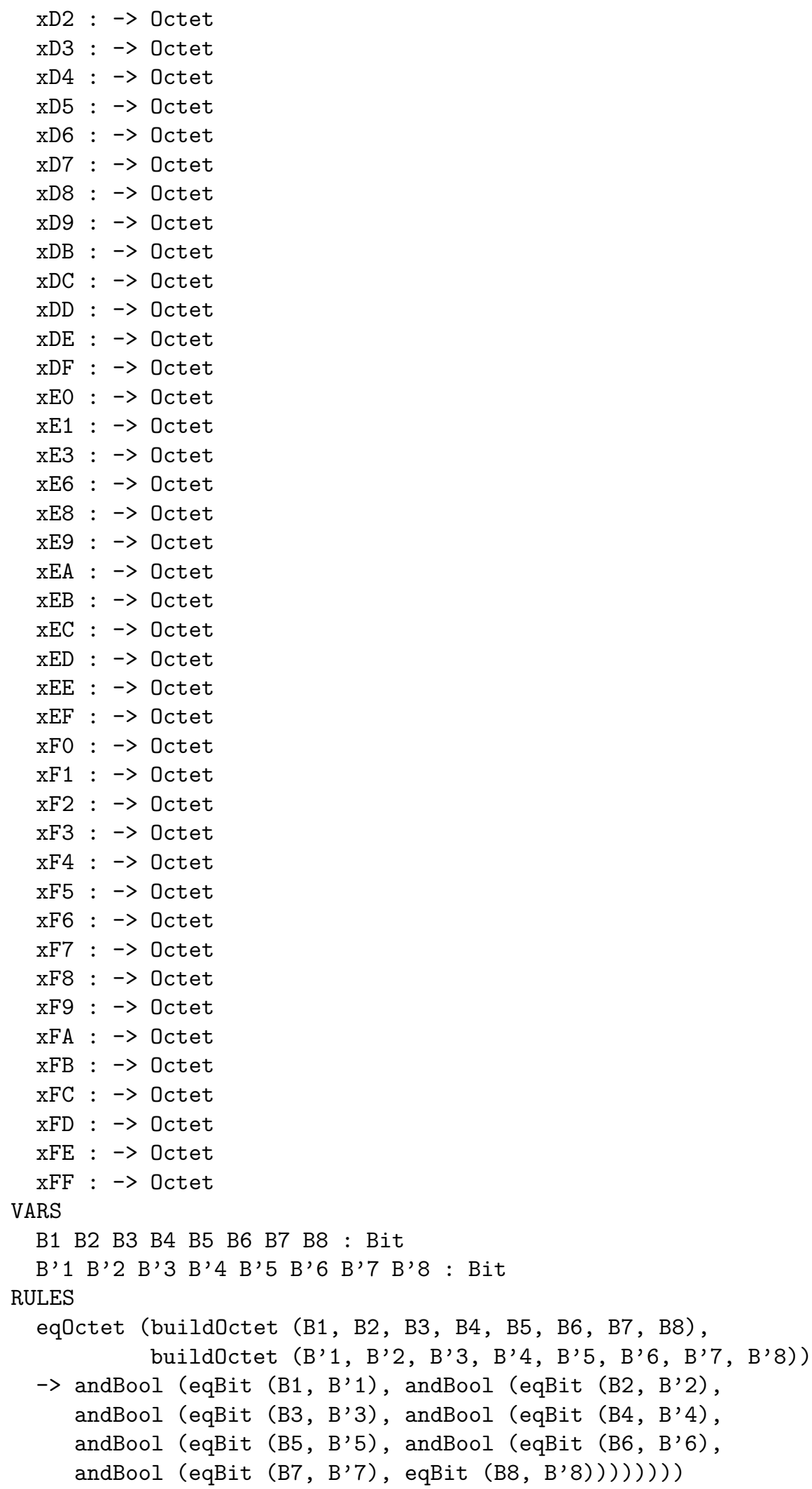




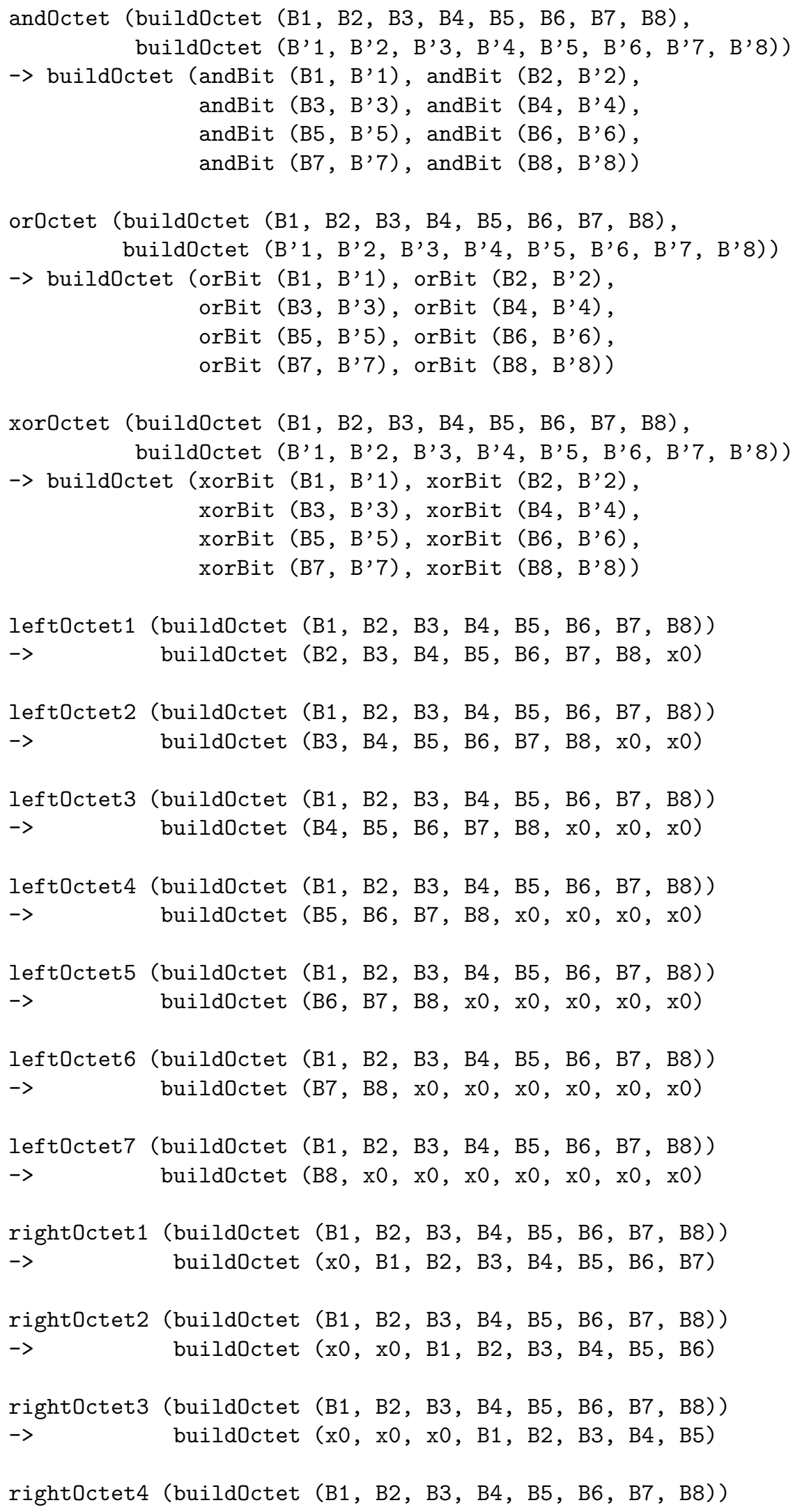




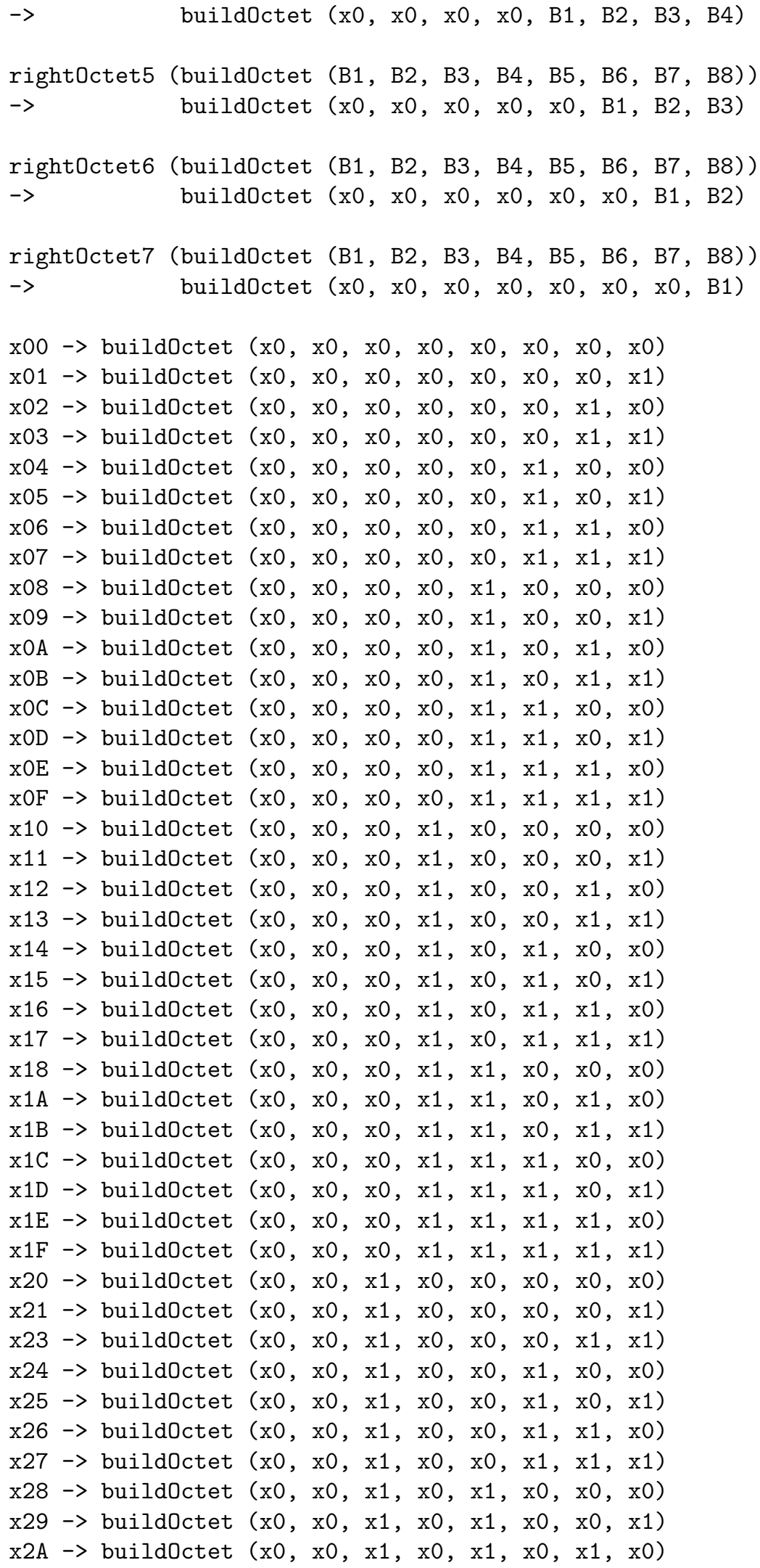




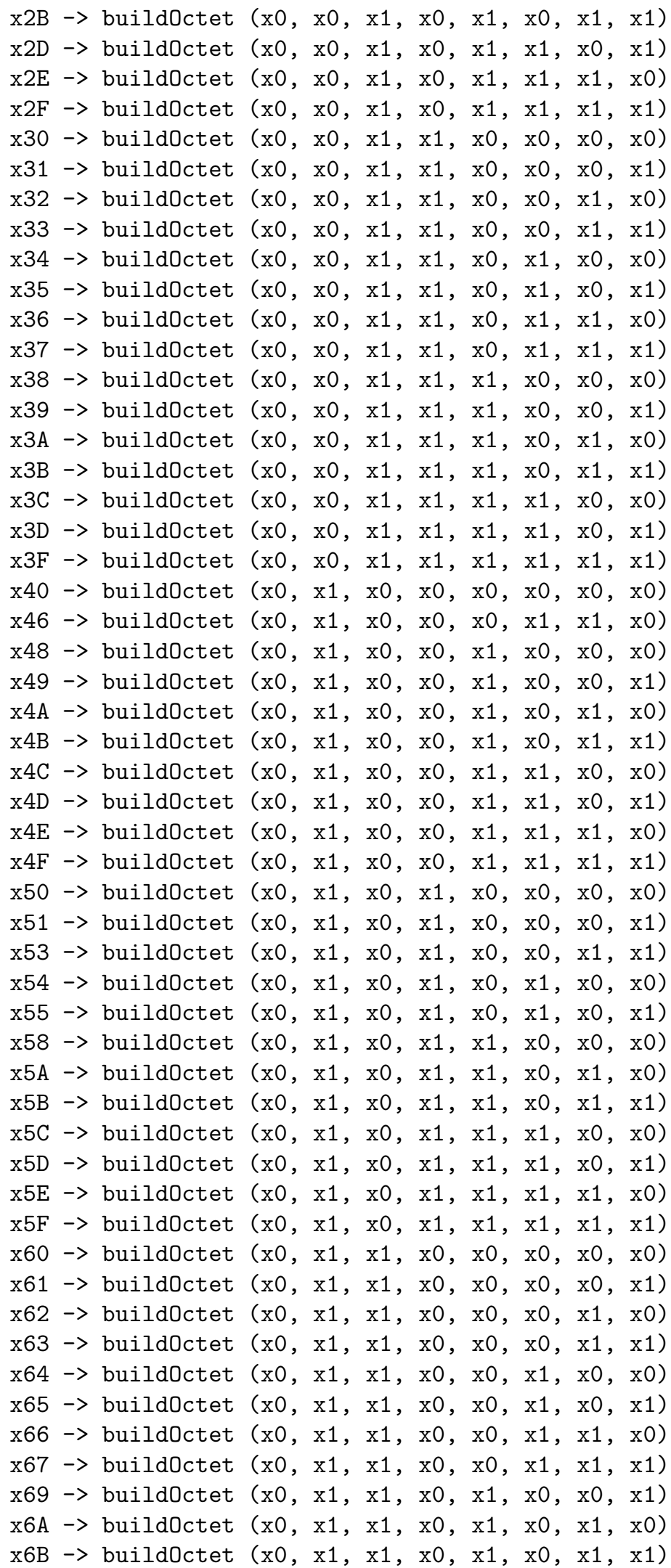




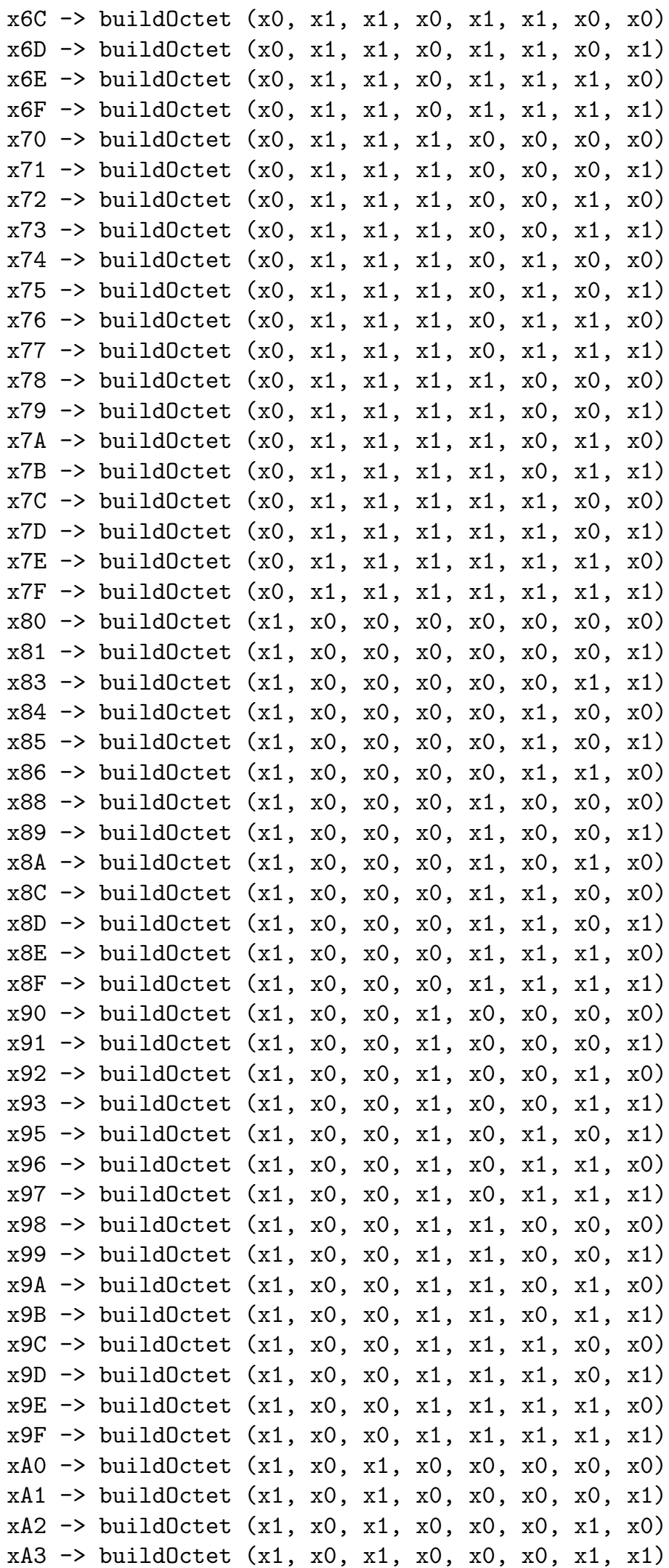


$\mathrm{xA4} \rightarrow>$ build0ctet ( $\mathrm{x} 1, \mathrm{x} 0, \mathrm{x} 1, \mathrm{x} 0, \mathrm{x} 0, \mathrm{x} 1, \mathrm{x} 0, \mathrm{x} 0$ ) $\mathrm{xA5} \rightarrow$ build0ctet $(\mathrm{x} 1, \mathrm{x} 0, \mathrm{x} 1, \mathrm{x} 0, \mathrm{x} 0, \mathrm{x} 1, \mathrm{x} 0, \mathrm{x} 1$ ) $\mathrm{xA6} \rightarrow$ build0ctet ( $\mathrm{x} 1, \mathrm{x} 0, \mathrm{x} 1, \mathrm{x} 0, \mathrm{x} 0, \mathrm{x} 1, \mathrm{x} 1, \mathrm{x} 0$ ) $\mathrm{xA7} \rightarrow$ build0ctet $(\mathrm{x} 1, \mathrm{x} 0, \mathrm{x} 1, \mathrm{x} 0, \mathrm{x} 0, \mathrm{x} 1, \mathrm{x} 1, \mathrm{x} 1)$ $\mathrm{xA8} \rightarrow$ build0ctet ( $\mathrm{x} 1, \mathrm{x} 0, \mathrm{x} 1, \mathrm{x} 0, \mathrm{x} 1, \mathrm{x} 0, \mathrm{x} 0, \mathrm{x} 0$ ) $\mathrm{xA9} \rightarrow$ build0ctet $(\mathrm{x} 1, \mathrm{x} 0, \mathrm{x} 1, \mathrm{x} 0, \mathrm{x} 1, \mathrm{x} 0, \mathrm{x} 0, \mathrm{x} 1)$ $\mathrm{xAA} \rightarrow$ build0ctet ( $\mathrm{x} 1, \mathrm{x} 0, \mathrm{x} 1, \mathrm{x} 0, \mathrm{x} 1, \mathrm{x} 0, \mathrm{x} 1, \mathrm{x} 0)$ $\mathrm{xAB} \rightarrow$ build0ctet ( $\mathrm{x} 1, \mathrm{x} 0, \mathrm{x} 1, \mathrm{x} 0, \mathrm{x} 1, \mathrm{x} 0, \mathrm{x} 1, \mathrm{x} 1$ ) $\mathrm{xAC} \rightarrow$ build0ctet ( $\mathrm{x} 1, \mathrm{x} 0, \mathrm{x} 1, \mathrm{x} 0, \mathrm{x} 1, \mathrm{x} 1, \mathrm{x} 0, \mathrm{x} 0$ ) $\mathrm{xAE} \rightarrow$ build0ctet ( $\mathrm{x} 1, \mathrm{x} 0, \mathrm{x} 1, \mathrm{x} 0, \mathrm{x} 1, \mathrm{x} 1, \mathrm{x} 1, \mathrm{x} 0$ ) $\mathrm{xAF} \rightarrow$ build0ctet ( $\mathrm{x} 1, \mathrm{x} 0, \mathrm{x} 1, \mathrm{x} 0, \mathrm{x} 1, \mathrm{x} 1, \mathrm{x} 1, \mathrm{x} 1$ ) $\mathrm{xB0} \rightarrow$ build0ctet ( $\mathrm{x} 1, \mathrm{x} 0, \mathrm{x} 1, \mathrm{x} 1, \mathrm{x} 0, \mathrm{x} 0, \mathrm{x} 0, \mathrm{x} 0$ ) $\mathrm{xB1} \rightarrow$ build0ctet ( $\mathrm{x} 1, \mathrm{x} 0, \mathrm{x} 1, \mathrm{x} 1, \mathrm{x} 0, \mathrm{x} 0, \mathrm{x} 0, \mathrm{x} 1$ ) $\mathrm{xB} 2 \rightarrow$ build0ctet ( $\mathrm{x} 1, \mathrm{x} 0, \mathrm{x} 1, \mathrm{x} 1, \mathrm{x} 0, \mathrm{x} 0, \mathrm{x} 1, \mathrm{x} 0$ ) $\mathrm{xB} 3 \rightarrow$ build0ctet $(\mathrm{x} 1, \mathrm{x} 0, \mathrm{x} 1, \mathrm{x} 1, \mathrm{x} 0, \mathrm{x} 0, \mathrm{x} 1, \mathrm{x} 1$ ) $\mathrm{xB5} \rightarrow$ build0ctet ( $\mathrm{x} 1, \mathrm{x} 0, \mathrm{x} 1, \mathrm{x} 1, \mathrm{x} 0, \mathrm{x} 1, \mathrm{x} 0, \mathrm{x} 1$ ) $\mathrm{xB} 6 \rightarrow$ build0ctet ( $\mathrm{x} 1, \mathrm{x} 0, \mathrm{x} 1, \mathrm{x} 1, \mathrm{x} 0, \mathrm{x} 1, \mathrm{x} 1, \mathrm{x} 0$ ) $\mathrm{xB} 8 \rightarrow$ build0ctet ( $\mathrm{x} 1, \mathrm{x} 0, \mathrm{x} 1, \mathrm{x} 1, \mathrm{x} 1, \mathrm{x} 0, \mathrm{x} 0, \mathrm{x} 0$ ) $\mathrm{xB9} \rightarrow$ build0ctet ( $\mathrm{x} 1, \mathrm{x} 0, \mathrm{x} 1, \mathrm{x} 1, \mathrm{x} 1, \mathrm{x} 0, \mathrm{x} 0, \mathrm{x} 1)$ $\mathrm{xBA} \rightarrow$ build0ctet ( $\mathrm{x} 1, \mathrm{x} 0, \mathrm{x} 1, \mathrm{x} 1, \mathrm{x} 1, \mathrm{x} 0, \mathrm{x} 1, \mathrm{x} 0$ ) $\mathrm{xBB} \rightarrow$ build0ctet ( $\mathrm{x} 1, \mathrm{x} 0, \mathrm{x} 1, \mathrm{x} 1, \mathrm{x} 1, \mathrm{x} 0, \mathrm{x} 1, \mathrm{x} 1$ ) $\mathrm{xBC} \rightarrow$ build0ctet ( $\mathrm{x} 1, \mathrm{x} 0, \mathrm{x} 1, \mathrm{x} 1, \mathrm{x} 1, \mathrm{x} 1, \mathrm{x} 0, \mathrm{x} 0$ ) $\mathrm{xBE} \rightarrow$ build0ctet ( $\mathrm{x} 1, \mathrm{x} 0, \mathrm{x} 1, \mathrm{x} 1, \mathrm{x} 1, \mathrm{x} 1, \mathrm{x} 1, \mathrm{x} 0$ ) $\mathrm{xBF} \rightarrow$ build0ctet ( $\mathrm{x} 1, \mathrm{x} 0, \mathrm{x} 1, \mathrm{x} 1, \mathrm{x} 1, \mathrm{x} 1, \mathrm{x} 1, \mathrm{x} 1)$ $\mathrm{xC0} \rightarrow$ build0ctet ( $\mathrm{x} 1, \mathrm{x} 1, \mathrm{x} 0, \mathrm{x} 0, \mathrm{x} 0, \mathrm{x} 0, \mathrm{x} 0, \mathrm{x} 0$ ) $\mathrm{xC1} \rightarrow$ build0ctet ( $\mathrm{x} 1, \mathrm{x} 1, \mathrm{x} 0, \mathrm{x} 0, \mathrm{x} 0, \mathrm{x} 0, \mathrm{x} 0, \mathrm{x} 1$ ) $\mathrm{xC2} \rightarrow$ build0ctet ( $\mathrm{x} 1, \mathrm{x} 1, \mathrm{x} 0, \mathrm{x} 0, \mathrm{x} 0, \mathrm{x} 0, \mathrm{x} 1, \mathrm{x} 0$ ) $\mathrm{xC4} \rightarrow$ build0ctet ( $\mathrm{x} 1, \mathrm{x} 1, \mathrm{x} 0, \mathrm{x} 0, \mathrm{x} 0, \mathrm{x} 1, \mathrm{x} 0, \mathrm{x} 0$ ) $\mathrm{xC5} \rightarrow$ build0ctet ( $\mathrm{x} 1, \mathrm{x} 1, \mathrm{x} 0, \mathrm{x} 0, \mathrm{x} 0, \mathrm{x} 1, \mathrm{x} 0, \mathrm{x} 1$ ) $\mathrm{xC6} \rightarrow$ build0ctet ( $\mathrm{x} 1, \mathrm{x} 1, \mathrm{x} 0, \mathrm{x} 0, \mathrm{x} 0, \mathrm{x} 1, \mathrm{x} 1, \mathrm{x} 0$ ) $\mathrm{xC7} \rightarrow$ build0ctet ( $\mathrm{x} 1, \mathrm{x} 1, \mathrm{x} 0, \mathrm{x} 0, \mathrm{x} 0, \mathrm{x} 1, \mathrm{x} 1, \mathrm{x} 1)$ $\mathrm{xC8} \rightarrow$ build0ctet ( $\mathrm{x} 1, \mathrm{x} 1, \mathrm{x} 0, \mathrm{x} 0, \mathrm{x} 1, \mathrm{x} 0, \mathrm{x} 0, \mathrm{x} 0$ ) $\mathrm{xC9} \rightarrow$ build0ctet ( $\mathrm{x} 1, \mathrm{x} 1, \mathrm{x} 0, \mathrm{x} 0, \mathrm{x} 1, \mathrm{x} 0, \mathrm{x} 0, \mathrm{x} 1$ ) $\mathrm{xCA} \rightarrow$ build0ctet ( $\mathrm{x} 1, \mathrm{x} 1, \mathrm{x} 0, \mathrm{x} 0, \mathrm{x} 1, \mathrm{x} 0, \mathrm{x} 1, \mathrm{x} 0$ ) $\mathrm{xCB} \rightarrow$ build0ctet ( $\mathrm{x} 1, \mathrm{x} 1, \mathrm{x} 0, \mathrm{x} 0, \mathrm{x} 1, \mathrm{x} 0, \mathrm{x} 1, \mathrm{x} 1$ ) $\mathrm{xCC} \rightarrow$ build0ctet ( $\mathrm{x} 1, \mathrm{x} 1, \mathrm{x} 0, \mathrm{x} 0, \mathrm{x} 1, \mathrm{x} 1, \mathrm{x} 0, \mathrm{x} 0$ ) $\mathrm{xCD} \rightarrow$ build0ctet $(\mathrm{x} 1, \mathrm{x} 1, \mathrm{x} 0, \mathrm{x} 0, \mathrm{x} 1, \mathrm{x} 1, \mathrm{x} 0, \mathrm{x} 1$ ) $\mathrm{xCE} \rightarrow$ build0ctet ( $\mathrm{x} 1, \mathrm{x} 1, \mathrm{x} 0, \mathrm{x} 0, \mathrm{x} 1, \mathrm{x} 1, \mathrm{x} 1, \mathrm{x} 0$ ) $\mathrm{xD0} \rightarrow$ build0ctet ( $\mathrm{x} 1, \mathrm{x} 1, \mathrm{x} 0, \mathrm{x} 1, \mathrm{x} 0, \mathrm{x} 0, \mathrm{x} 0, \mathrm{x} 0$ ) $\mathrm{xD} 1 \rightarrow$ build0ctet $(\mathrm{x} 1, \mathrm{x} 1, \mathrm{x} 0, \mathrm{x} 1, \mathrm{x} 0, \mathrm{x} 0, \mathrm{x} 0, \mathrm{x} 1)$ $\mathrm{xD} 2 \rightarrow$ build0ctet ( $\mathrm{x} 1, \mathrm{x} 1, \mathrm{x} 0, \mathrm{x} 1, \mathrm{x} 0, \mathrm{x} 0, \mathrm{x} 1, \mathrm{x} 0$ ) $\mathrm{xD} 3 \rightarrow$ build0ctet ( $\mathrm{x} 1, \mathrm{x} 1, \mathrm{x} 0, \mathrm{x} 1, \mathrm{x} 0, \mathrm{x} 0, \mathrm{x} 1, \mathrm{x} 1$ ) $\mathrm{xD} 4 \rightarrow$ build0ctet ( $\mathrm{x} 1, \mathrm{x} 1, \mathrm{x} 0, \mathrm{x} 1, \mathrm{x} 0, \mathrm{x} 1, \mathrm{x} 0, \mathrm{x} 0$ ) $\mathrm{xD5} \rightarrow$ build0ctet ( $\mathrm{x} 1, \mathrm{x} 1, \mathrm{x} 0, \mathrm{x} 1, \mathrm{x} 0, \mathrm{x} 1, \mathrm{x} 0, \mathrm{x} 1)$ $\mathrm{xD6} \rightarrow$ build0ctet ( $\mathrm{x} 1, \mathrm{x} 1, \mathrm{x} 0, \mathrm{x} 1, \mathrm{x} 0, \mathrm{x} 1, \mathrm{x} 1, \mathrm{x} 0$ ) $\mathrm{xD} 7 \rightarrow$ build0ctet $(\mathrm{x} 1, \mathrm{x} 1, \mathrm{x} 0, \mathrm{x} 1, \mathrm{x} 0, \mathrm{x} 1, \mathrm{x} 1, \mathrm{x} 1)$ $\mathrm{xD} 8 \rightarrow$ build0ctet ( $\mathrm{x} 1, \mathrm{x} 1, \mathrm{x} 0, \mathrm{x} 1, \mathrm{x} 1, \mathrm{x} 0, \mathrm{x} 0, \mathrm{x} 0$ ) $\mathrm{xD} 9 \rightarrow$ build0ctet $(\mathrm{x} 1, \mathrm{x} 1, \mathrm{x} 0, \mathrm{x} 1, \mathrm{x} 1, \mathrm{x} 0, \mathrm{x} 0, \mathrm{x} 1)$ $\mathrm{xDB} \rightarrow$ build0ctet ( $\mathrm{x} 1, \mathrm{x} 1, \mathrm{x} 0, \mathrm{x} 1, \mathrm{x} 1, \mathrm{x} 0, \mathrm{x} 1, \mathrm{x} 1$ ) $\mathrm{xDC} \rightarrow$ build0ctet $(\mathrm{x} 1, \mathrm{x} 1, \mathrm{x} 0, \mathrm{x} 1, \mathrm{x} 1, \mathrm{x} 1, \mathrm{x} 0, \mathrm{x} 0$ ) $\mathrm{xDD} \rightarrow$ build0ctet ( $\mathrm{x} 1, \mathrm{x} 1, \mathrm{x} 0, \mathrm{x} 1, \mathrm{x} 1, \mathrm{x} 1, \mathrm{x} 0, \mathrm{x} 1$ ) $\mathrm{xDE} \rightarrow$ build0ctet $(\mathrm{x} 1, \mathrm{x} 1, \mathrm{x} 0, \mathrm{x} 1, \mathrm{x} 1, \mathrm{x} 1, \mathrm{x} 1, \mathrm{x} 0)$ 


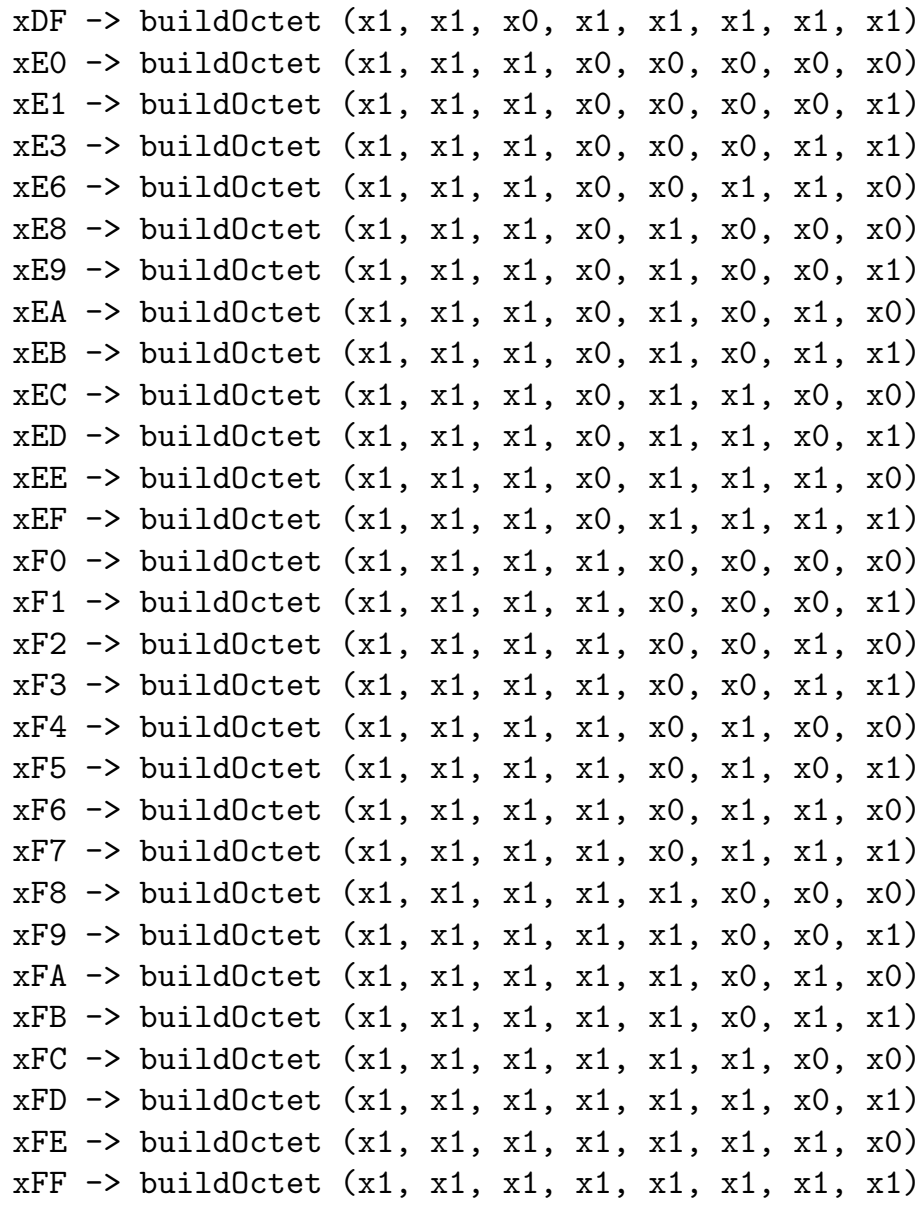

\section{B.5 Definitions for sort OctetSum}

We now define sort DctetSum that stores the result of the addition of two octets. Values of this sort are 9-bit words, made up using the constructor build0ctetSum that gathers one bit for the carry and an octet for the sum. The three principal non-constructors for this sort are eq0ctetSum (which tests equality), addOctetSum (which adds two octets and an input carry bit, and returns both an output carry bit and an 8-bit sum), and addOctet (which is derived from the former one by dropping the input and output carry bits); the other non-constructors are auxiliary functions implementing an 8-bit adder.

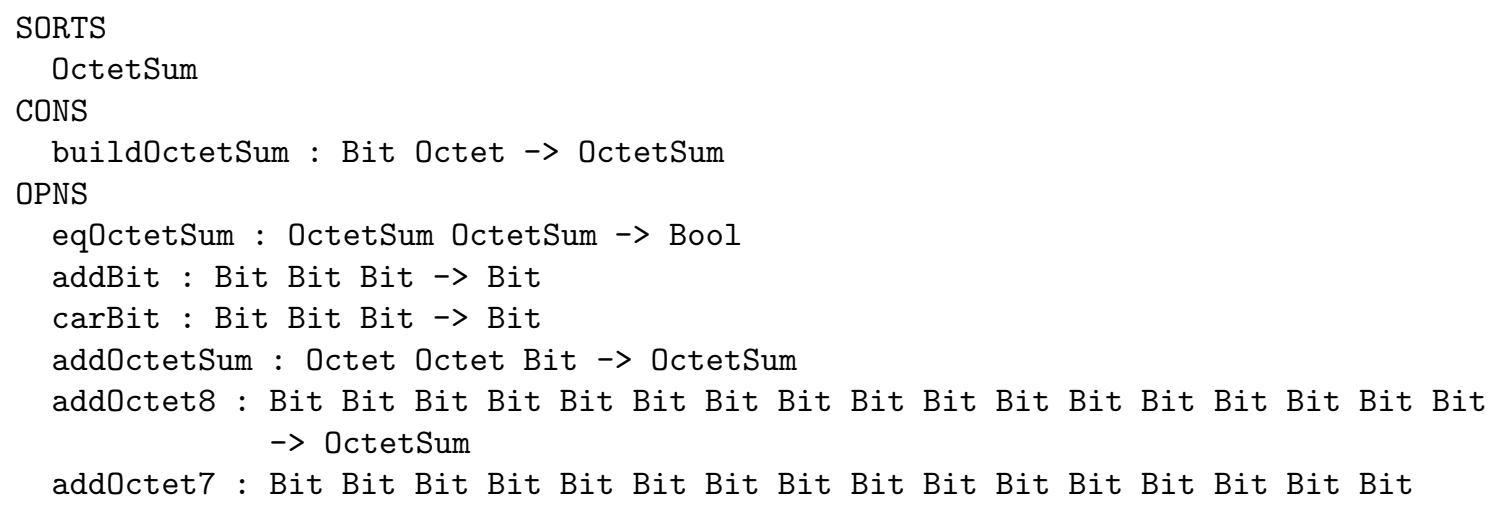




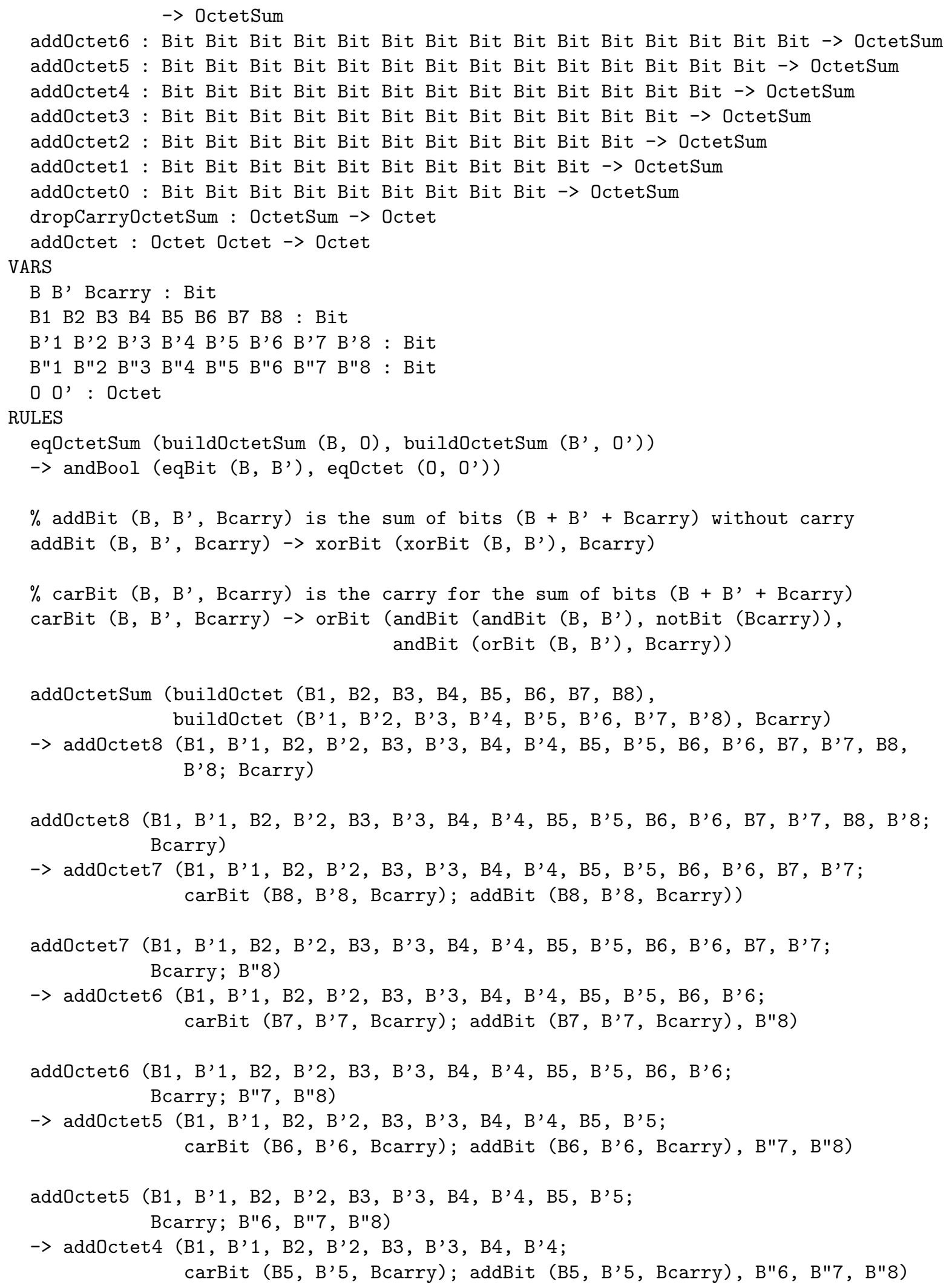




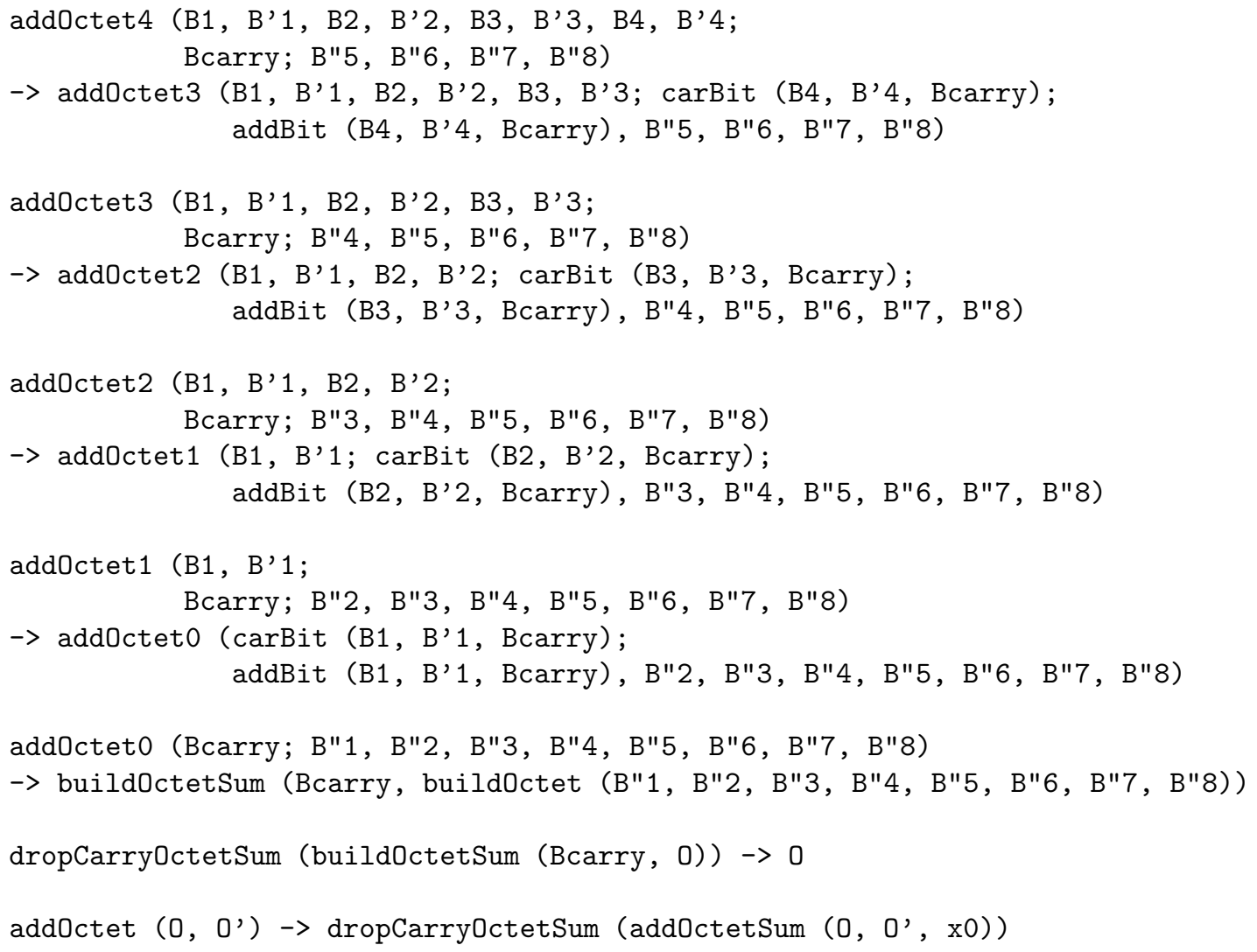

\section{B.6 Definitions for sort Half}

We now define 16-bit words (named "half words") using a constructor buildHalf that takes two octets and returns a half word, together with non-constructors implementing equality, two usual constants, and an operation mulOctet that takes two octets and computes their 16-bit product; the other nonconstructors are auxiliary functions implementing an 8-bit multiplier.

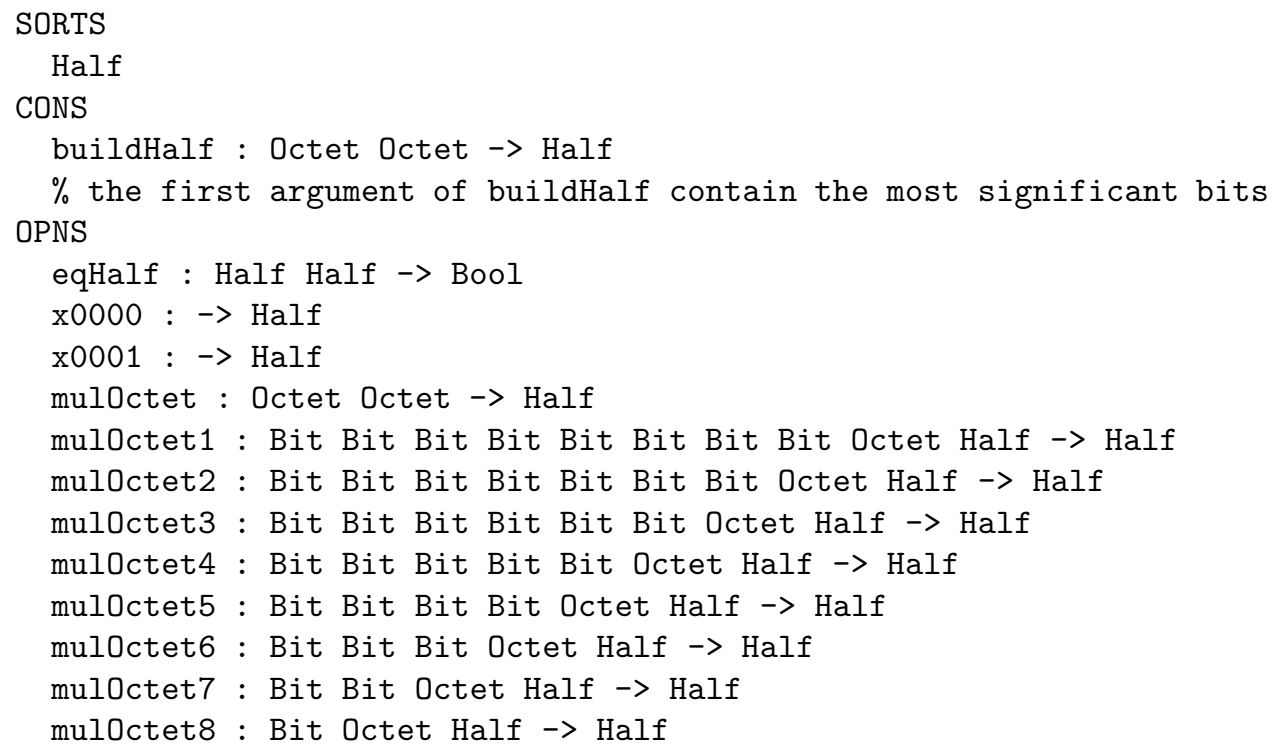




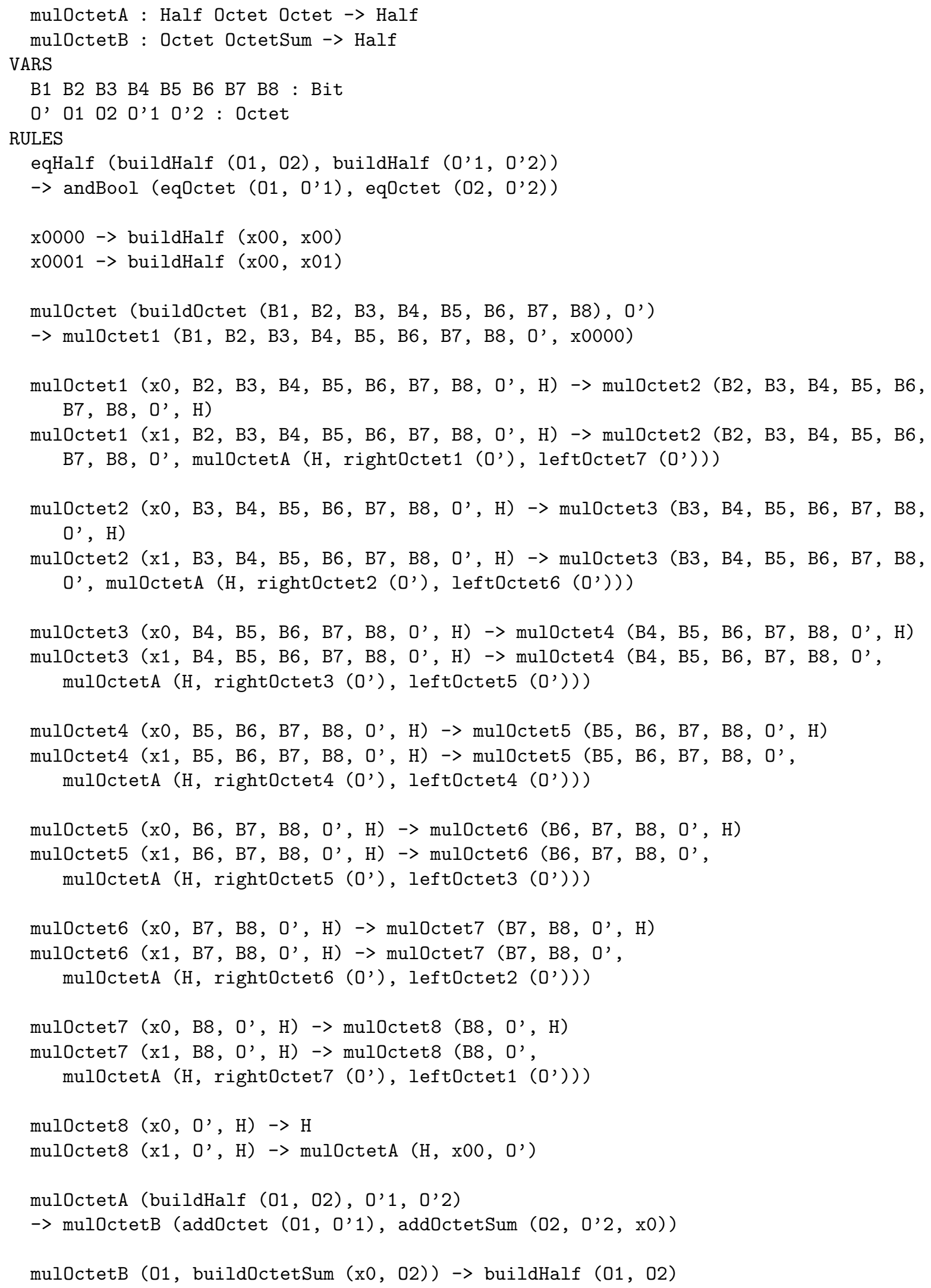


mulOctetB (01, buildOctetSum $(\mathrm{x} 1,02)) \rightarrow$ buildHalf (addOctet $(01, \mathrm{x} 01), 02)$

\section{B.7 Definitions for sort HalfSum}

We now define sort HalfSum that stores the result of the addition of two half words. Values of this sort are 17-bit words, made up using the constructor buildHalfSum that gathers one bit for the carry and a half word for the sum. The five principal non-constructors for this sort are eqHalfSum (which tests equality), addHalfSum (which adds two half words and returns both a carry bit and a 16-bit sum), addHalf (which is derived from the former one by dropping the carry bit), addHalfOctet and addHalfOctets (which are similar to the former one but take octet arguments that are converted to half words before summation); the other non-constructors are auxiliary functions implementing a 16-bit adder built using two 8-bit adders.

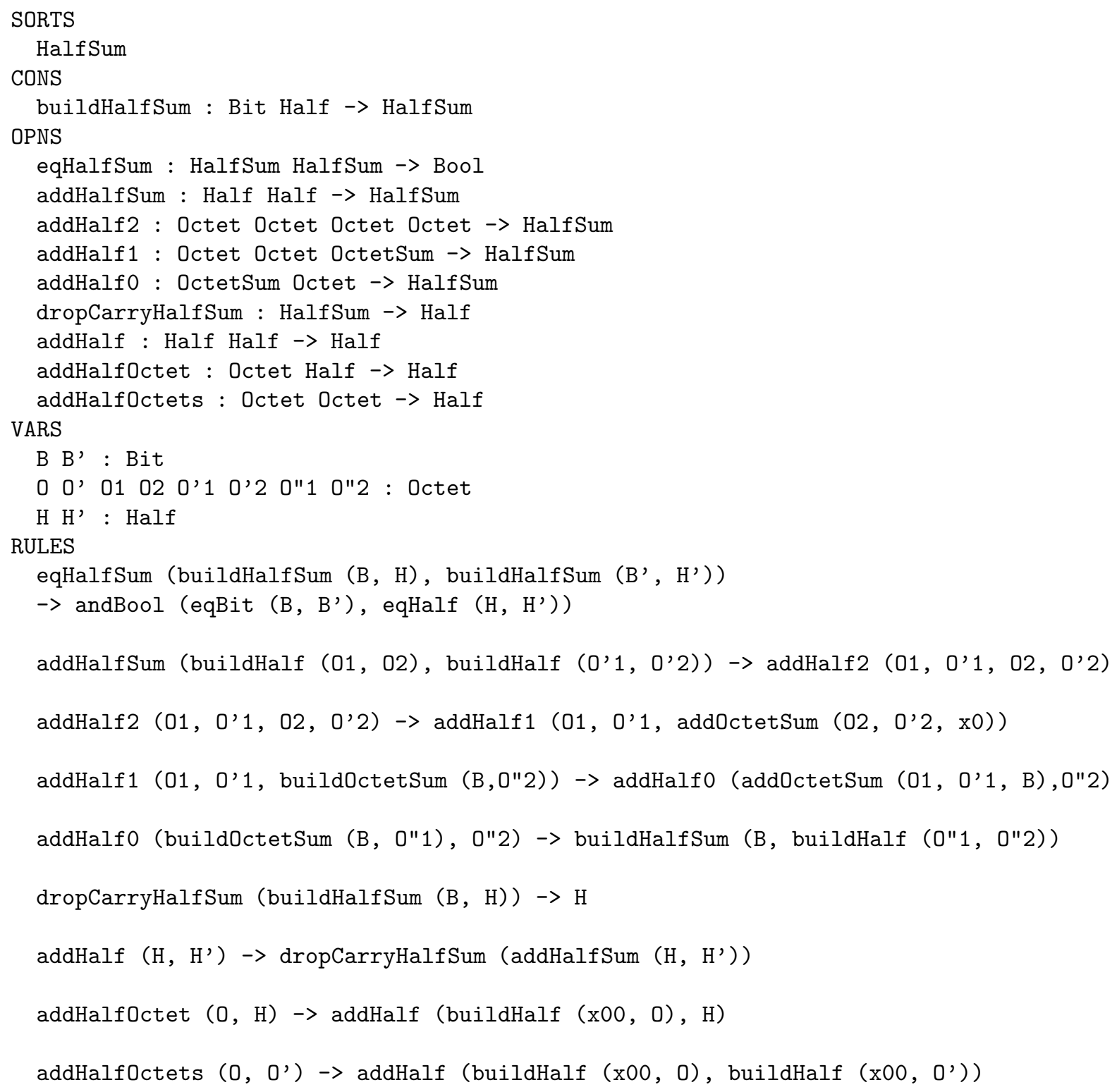




\section{B.8 Definitions for sort Block}

We now define 32-bit words (named "blocks" according to the MAA terminology) using a constructor buildBlock that takes four octets and returns a block. The seven principal non-constructors for this sort are eqBlock (which tests equality), andBlock, orBlock, and xorBlock (which implement bitwise logical operations on blocks), HalfU and HalfL (which decompose a block into two half words), and mulHalf (which takes two half words and computes their 32-bit product); the other non-constructors are auxiliary functions implementing a 16-bit multiplier built using four 8-bit multipliers, as well as all block constants needed to formally describe the MAA and its test vectors.

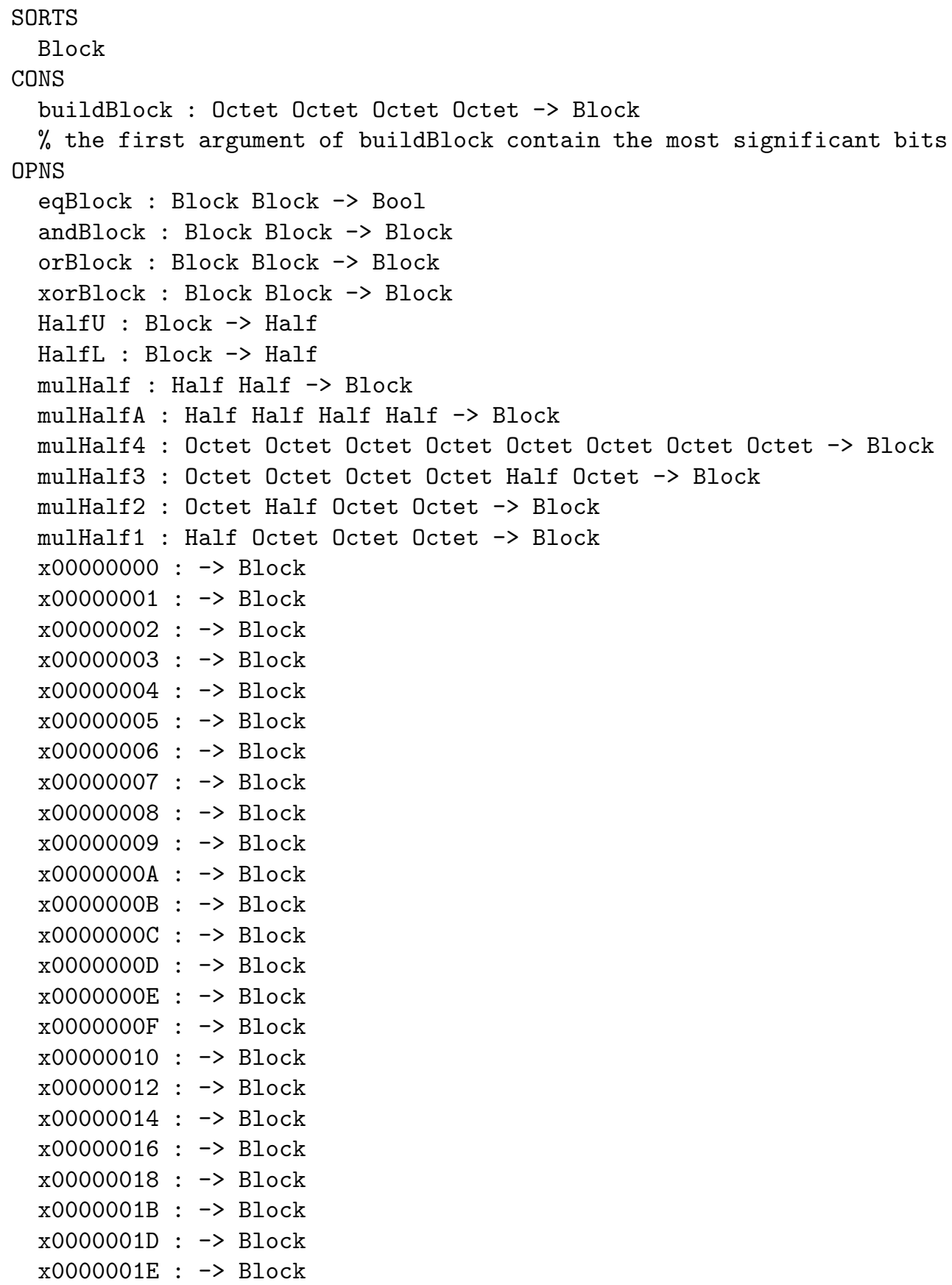




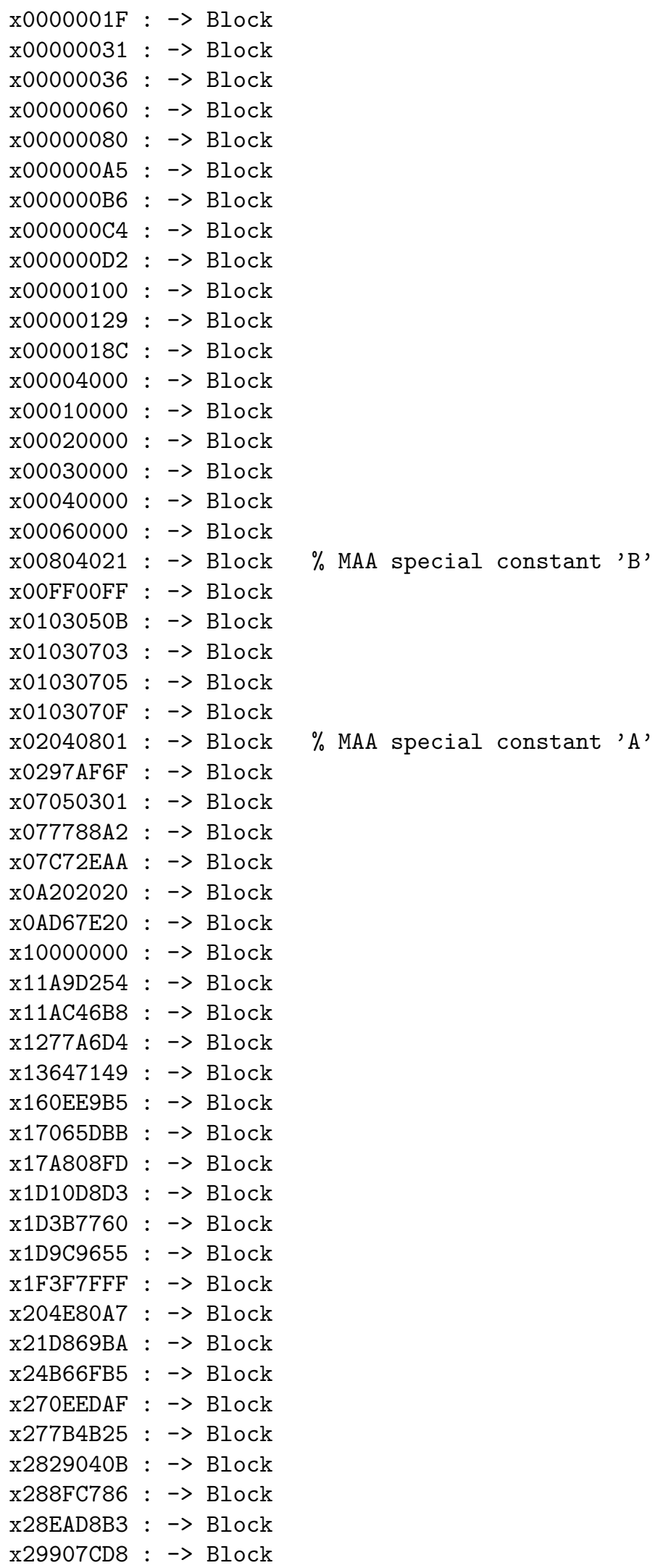

$\%$ MAA special constant 'B'

$\%$ MAA special constant 'A' 


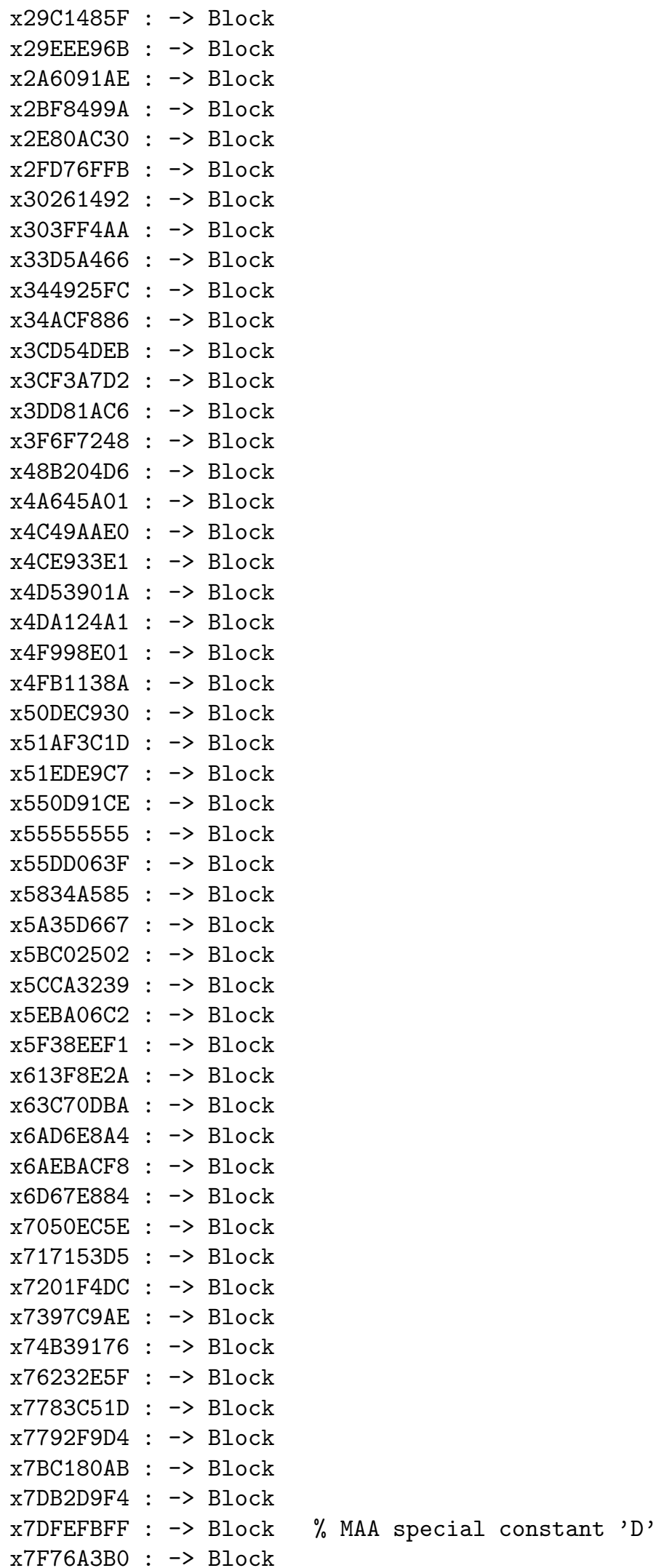




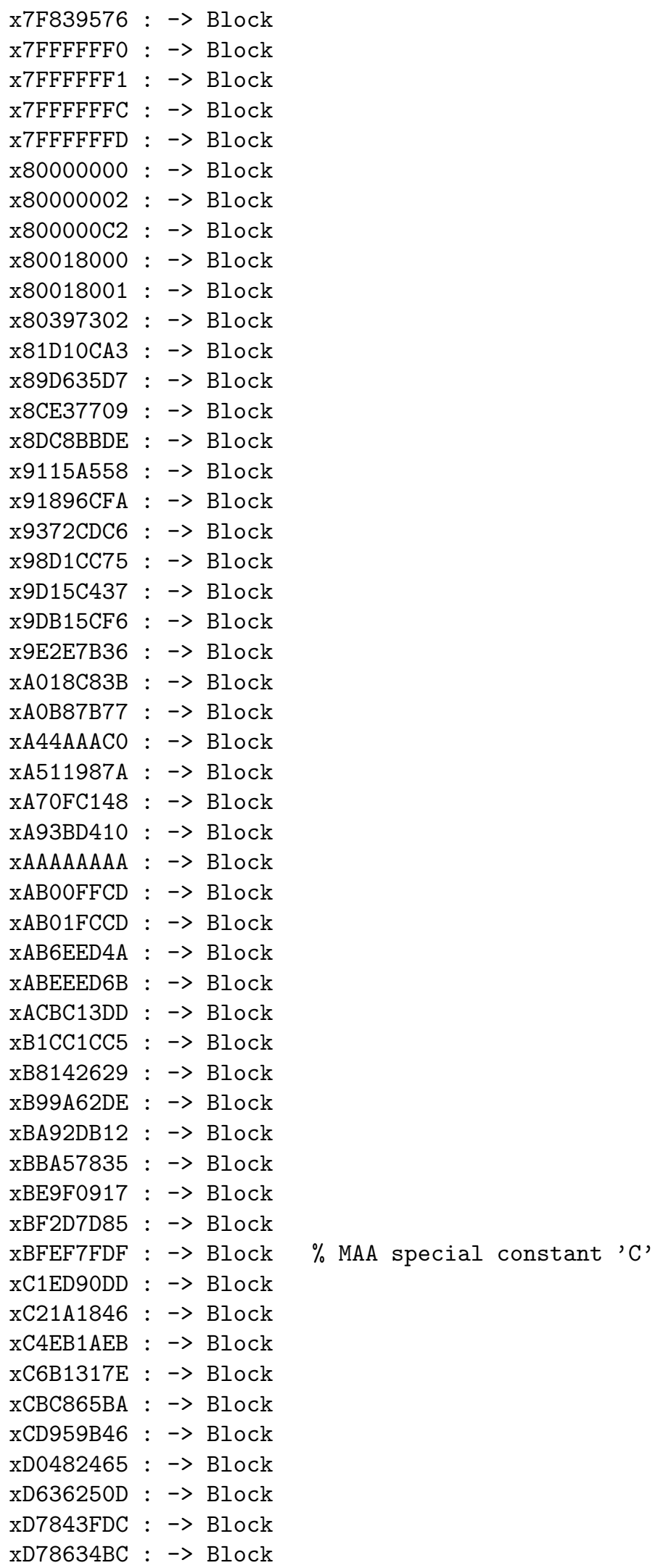




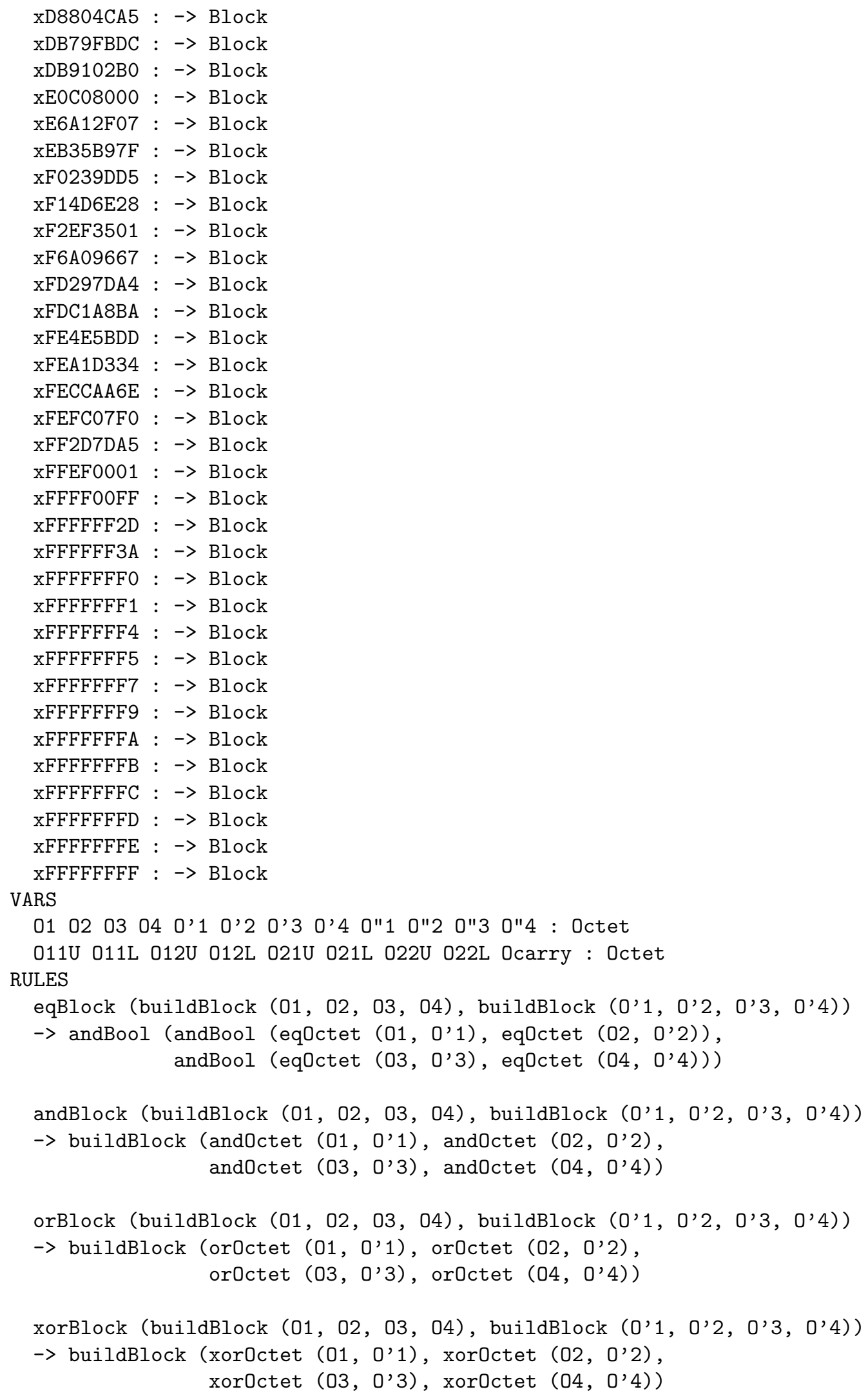




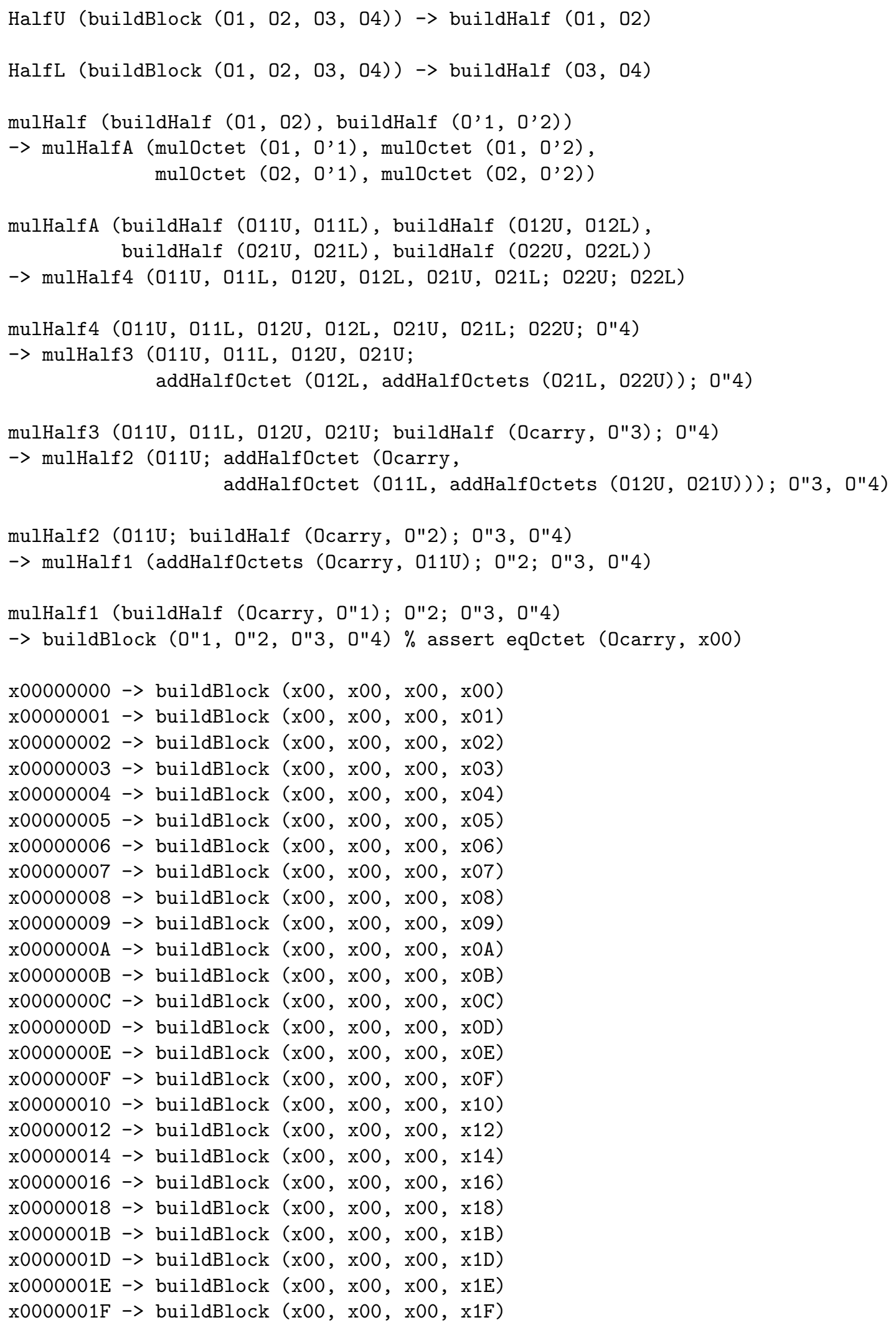




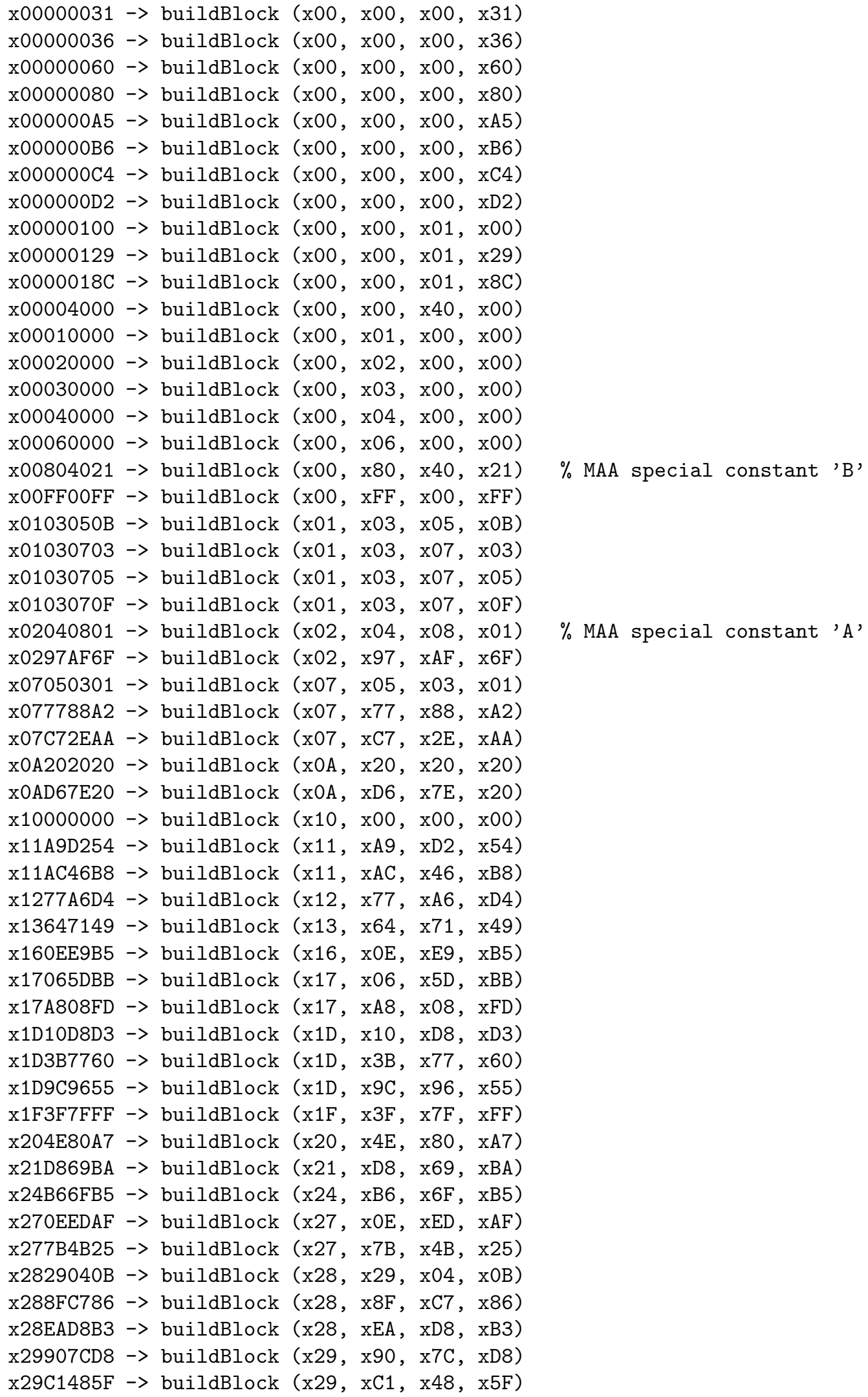

$\%$ MAA special constant ' $B$ '

$\%$ MAA special constant 'A' 


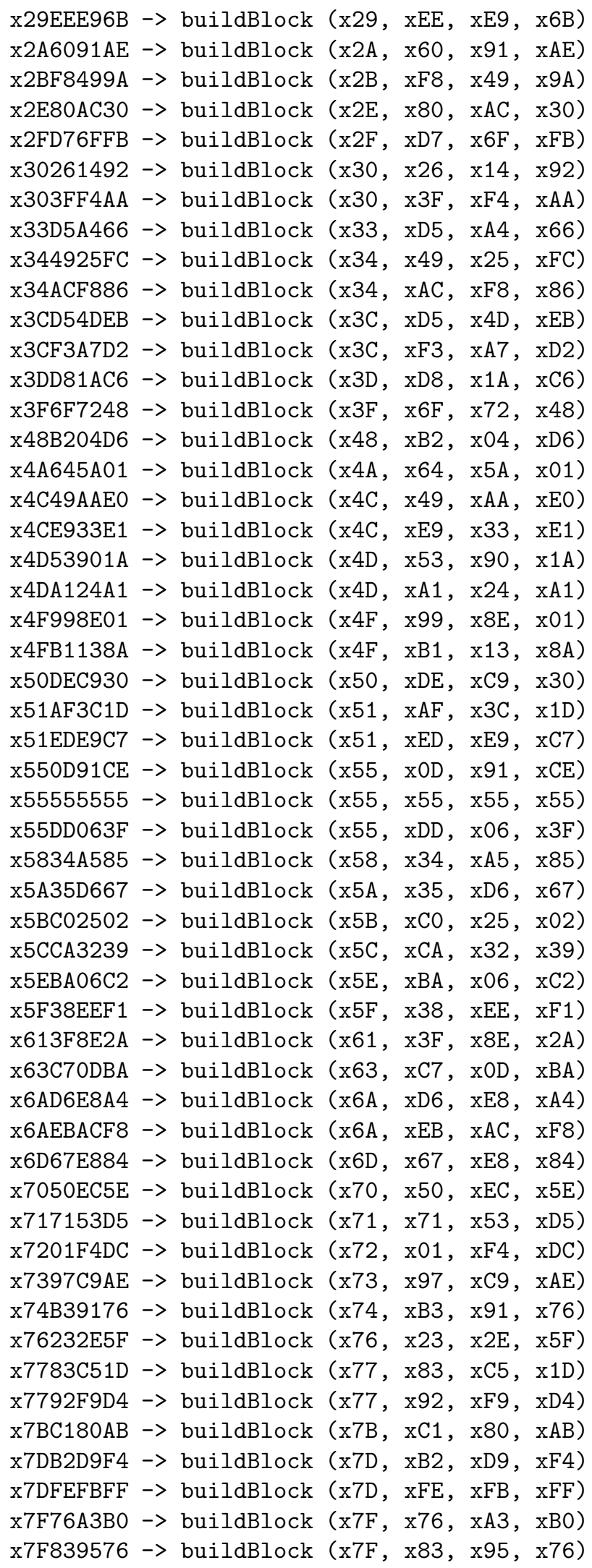




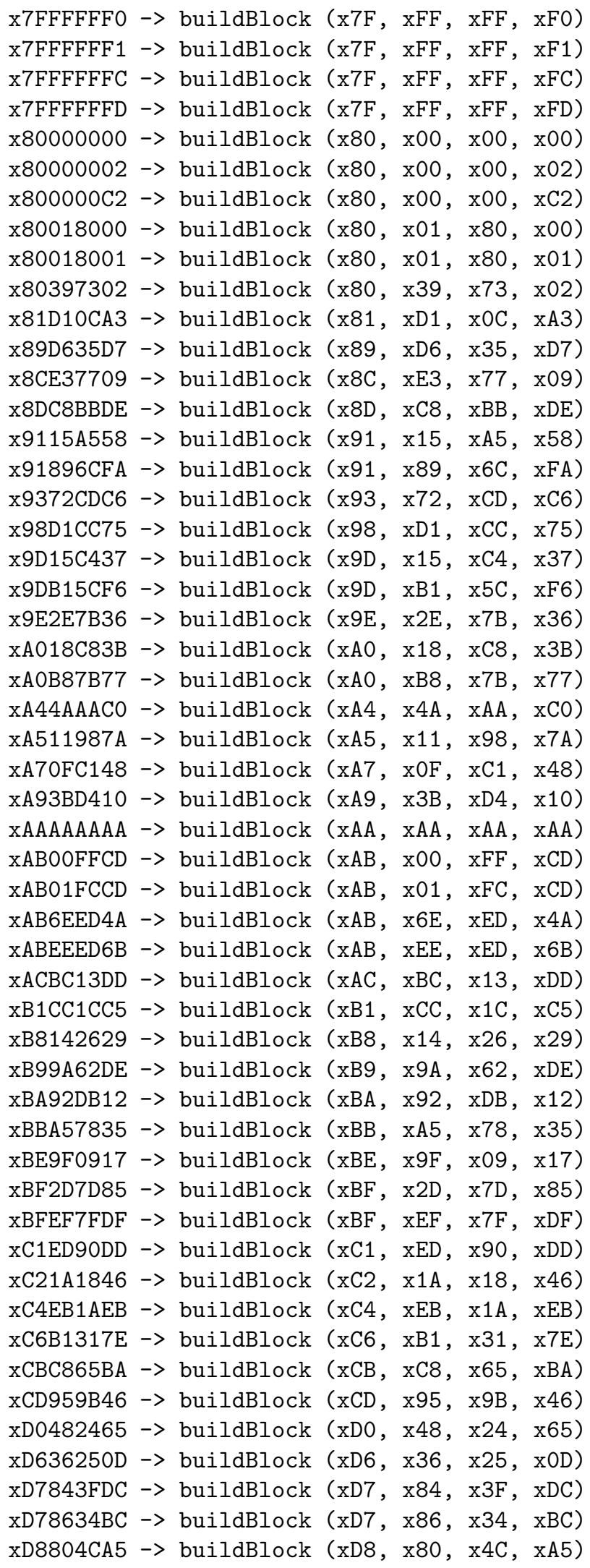

$\%$ MAA special constant 'C' 


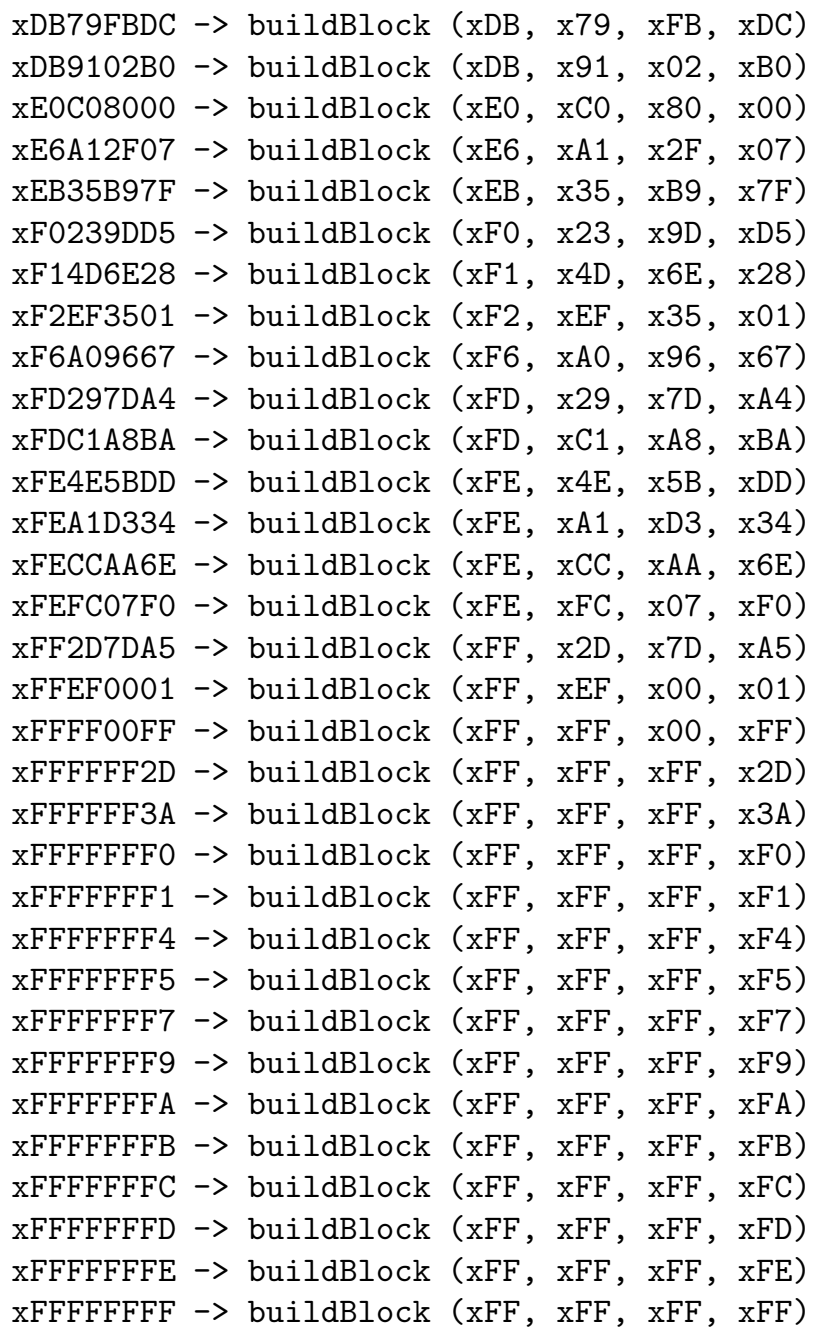

\section{B.9 Definitions for sort BlockSum}

We now define sort BlockSum that stores the result of the addition of two blocks. Values of this sort are 33-bit words, made up using the constructor buildBlockSum that gathers one bit for the carry and a block for the sum. The five principal non-constructors for this sort are eqBlockSum (which tests equality), addBlockSum (which adds two blocks and returns both a carry bit and a 32-bit sum), addBlock (which is derived from the former one by dropping the carry bit), addBlockHalf and addBlockHalves (which are similar to the former one but take half-word arguments that are converted to blocks before summation); the other non-constructors are auxiliary functions implementing a 32-bit adder built using four 8-bit adders.

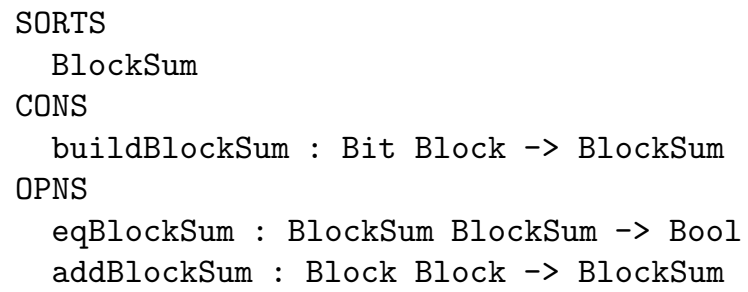




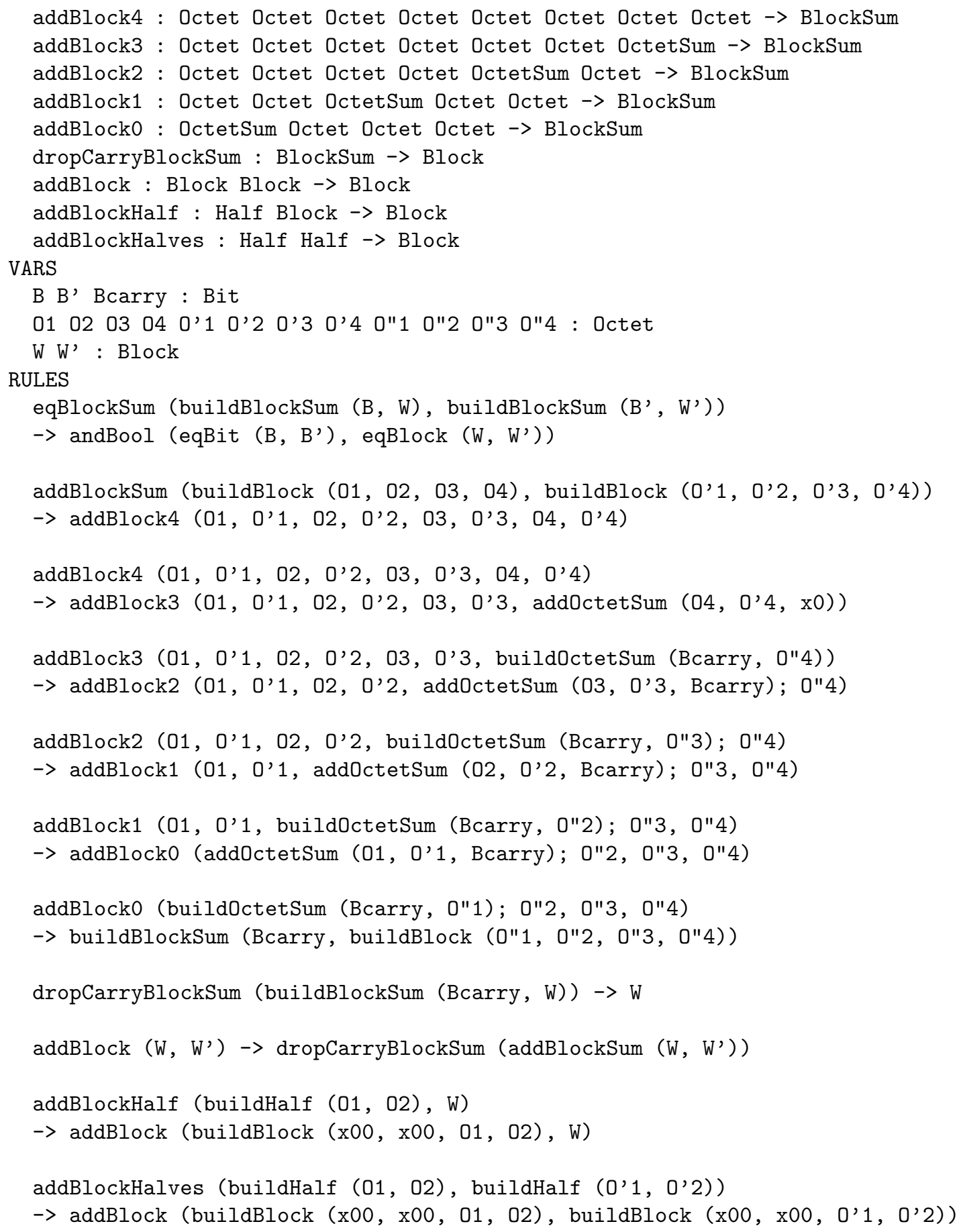

\section{B.10 Definitions for sort Pair}

We now define 64-bit words (named "pairs" according to the MAA terminology) using a constructor buildPair that takes two blocks and returns a pair. The two principal non-constructors for this sort are eqPair (which tests equality) and mulBlock (which takes two blocks and computes their 64-bit product); the other non-constructors are auxiliary functions implementing a 32-bit multiplier built using 
four 16-bit multipliers.

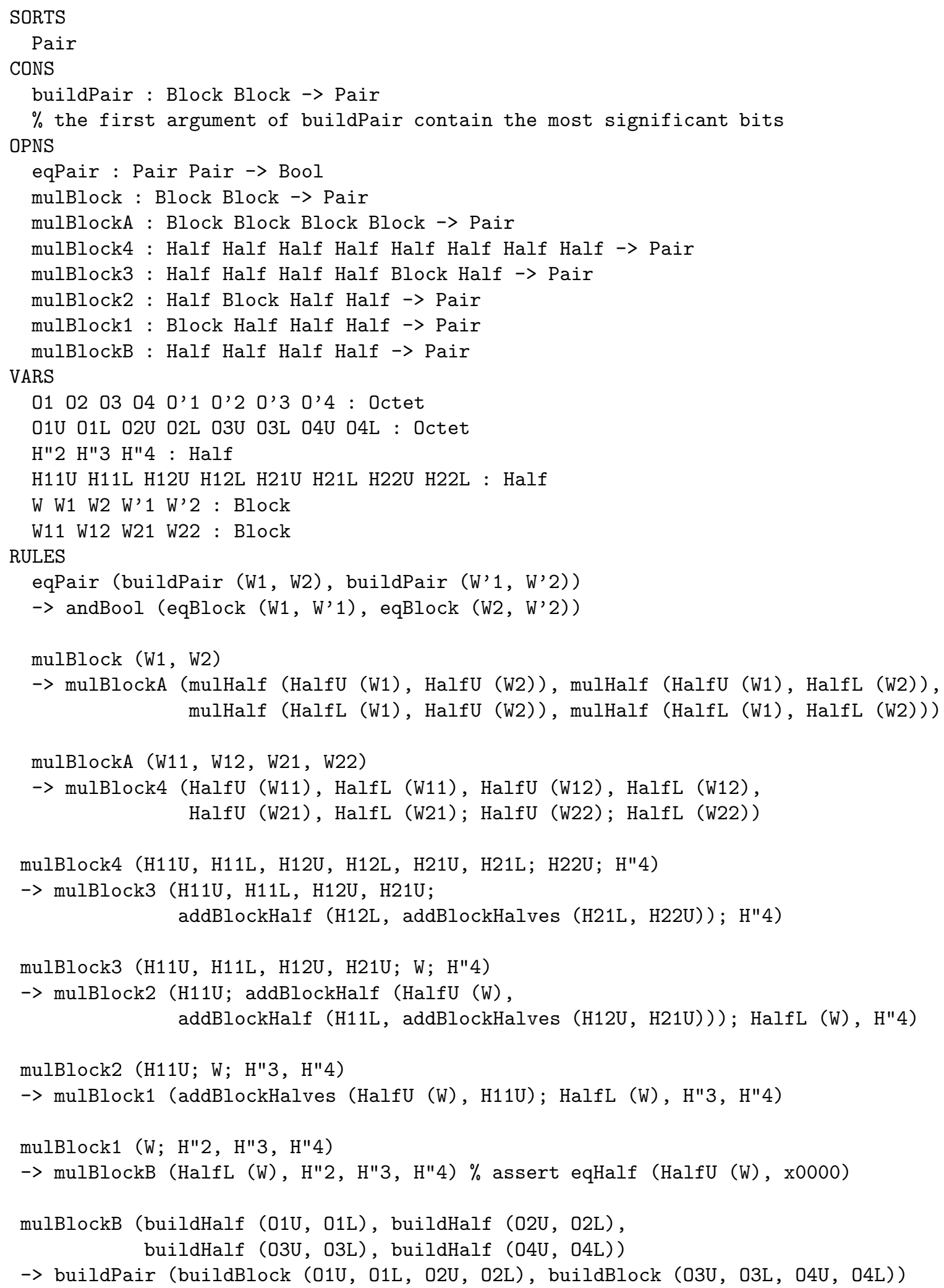




\section{B.11 Definitions for sort Key}

We now define a sort Key that is intended to represent the 64-bit keys $(J, K)$ used by the MAA. This sort has a constructor buildKey that takes two blocks and returns a key. In [15], keys are represented using the sort Pair, but we prefer introducing a dedicated sort to clearly distinguish between keys and, e.g., results of the multiplication of two blocks.

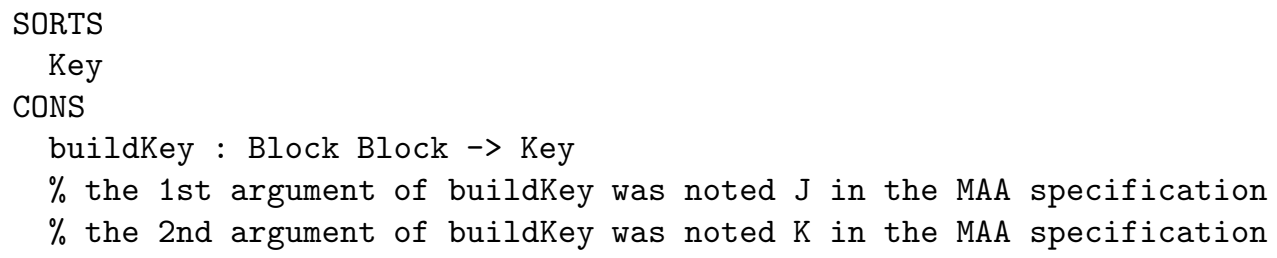

\section{B.12 Definitions for sort Message}

We now define messages, which are non-empty lists of blocks built using two constructors unitMessage and consMessage; there are three non-constructors for this sort: appendMessage (which inserts a block at the end of a list), reverseMessage (which reverses a list), and makeMessage (which generates a message of a given length, the blocks of which follow an arithmetic progression).

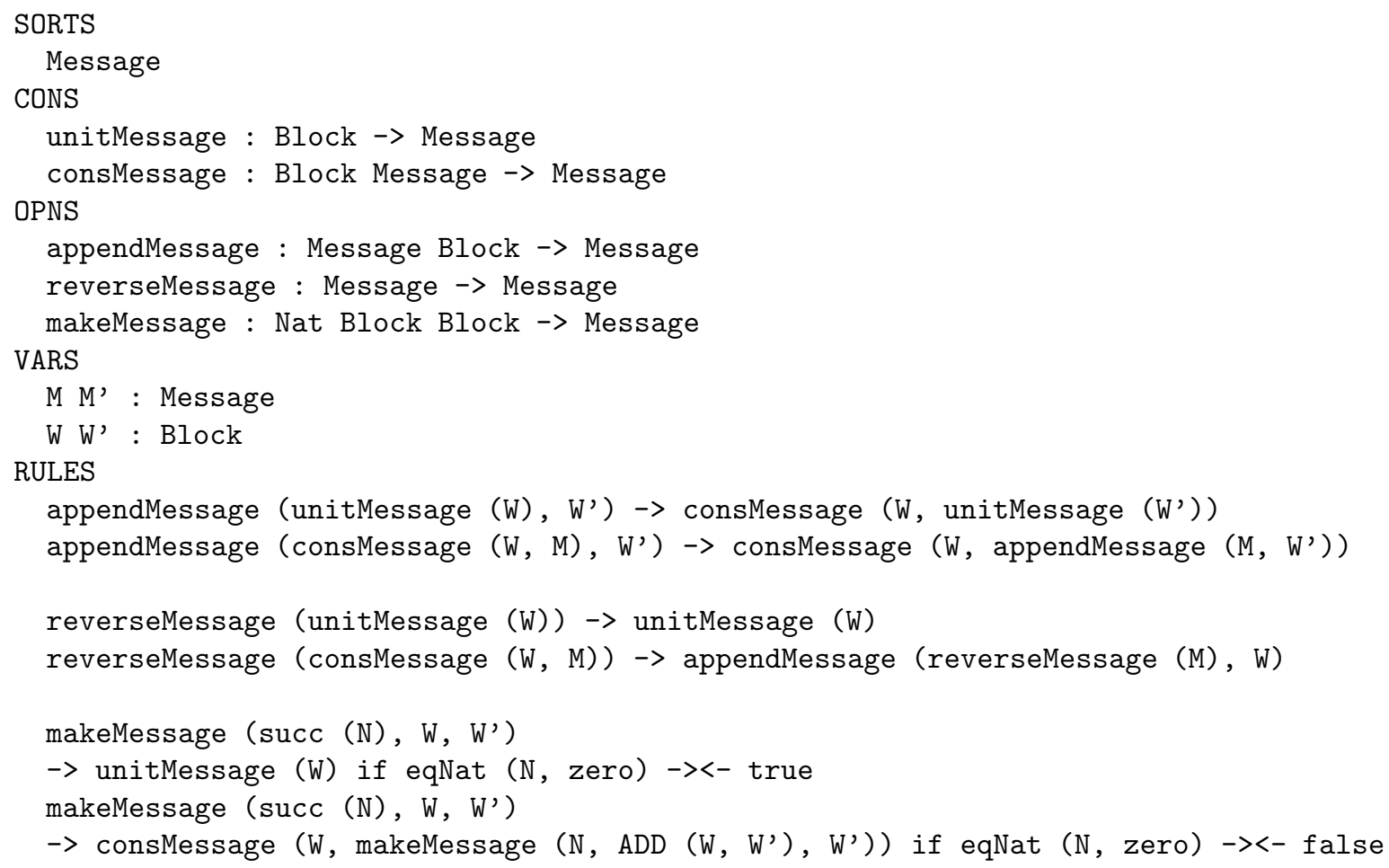

If needed, the two conditional rules could be eliminated by modifying the definition of makeMessage as follows:

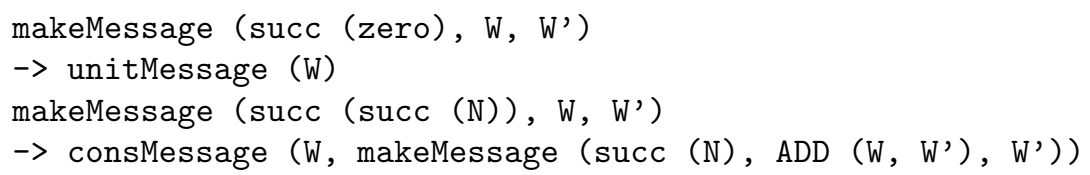




\section{B.13 Definitions for sort SegmentedMessage}

We now define segmented messages, which are non-empty lists of messages, each message containing up to 1204 octets (i.e., 256 blocks). Values of this sort are built using two constructors unitSegment and consSegment; the principal non-constructor is splitSegment, which converts a message into a segmented message.

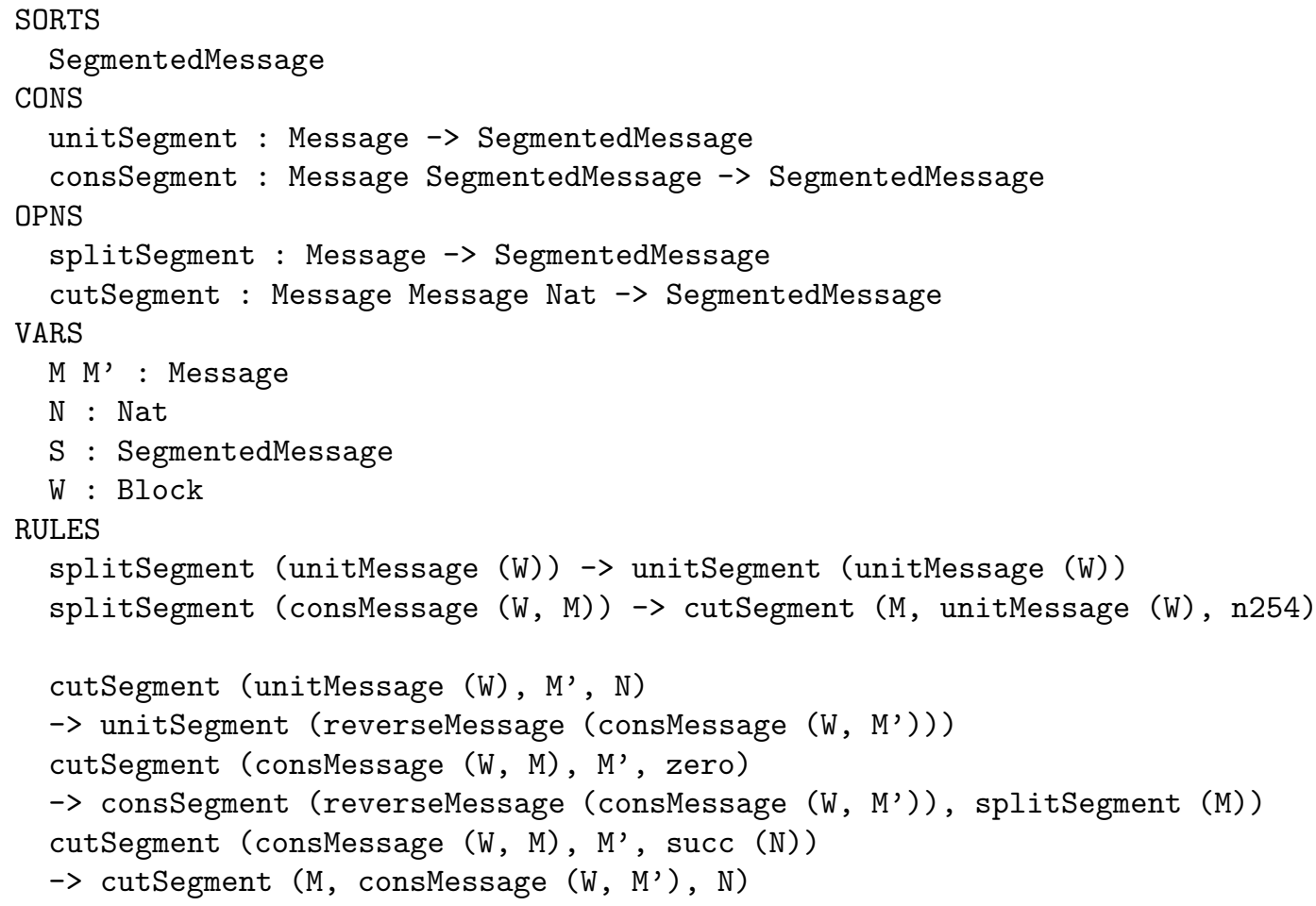

\section{B.14 Definitions (1) of MAA-specific cryptographic functions}

We now define a first set of functions to be used for MAA computations, most of which were present in [2] or have been later introduced in [15]. Operations ADD, AND, MUL, OR, and XOR are merely aliases of already-defined functions on Blocks; operations BYT' and ADDC' are just auxiliary functions.

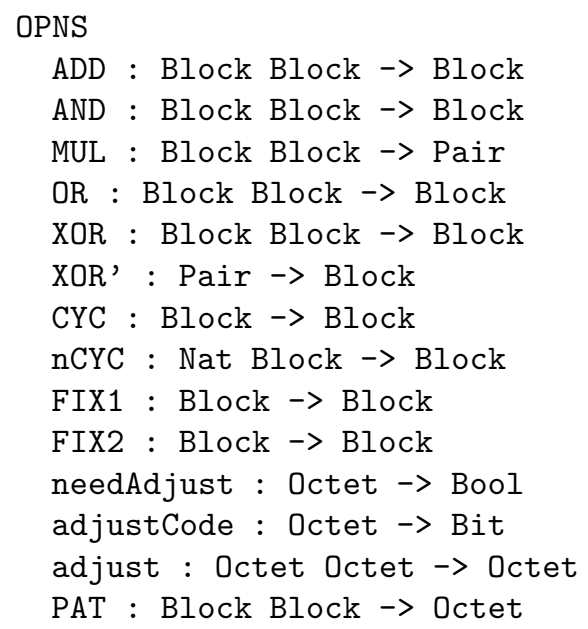




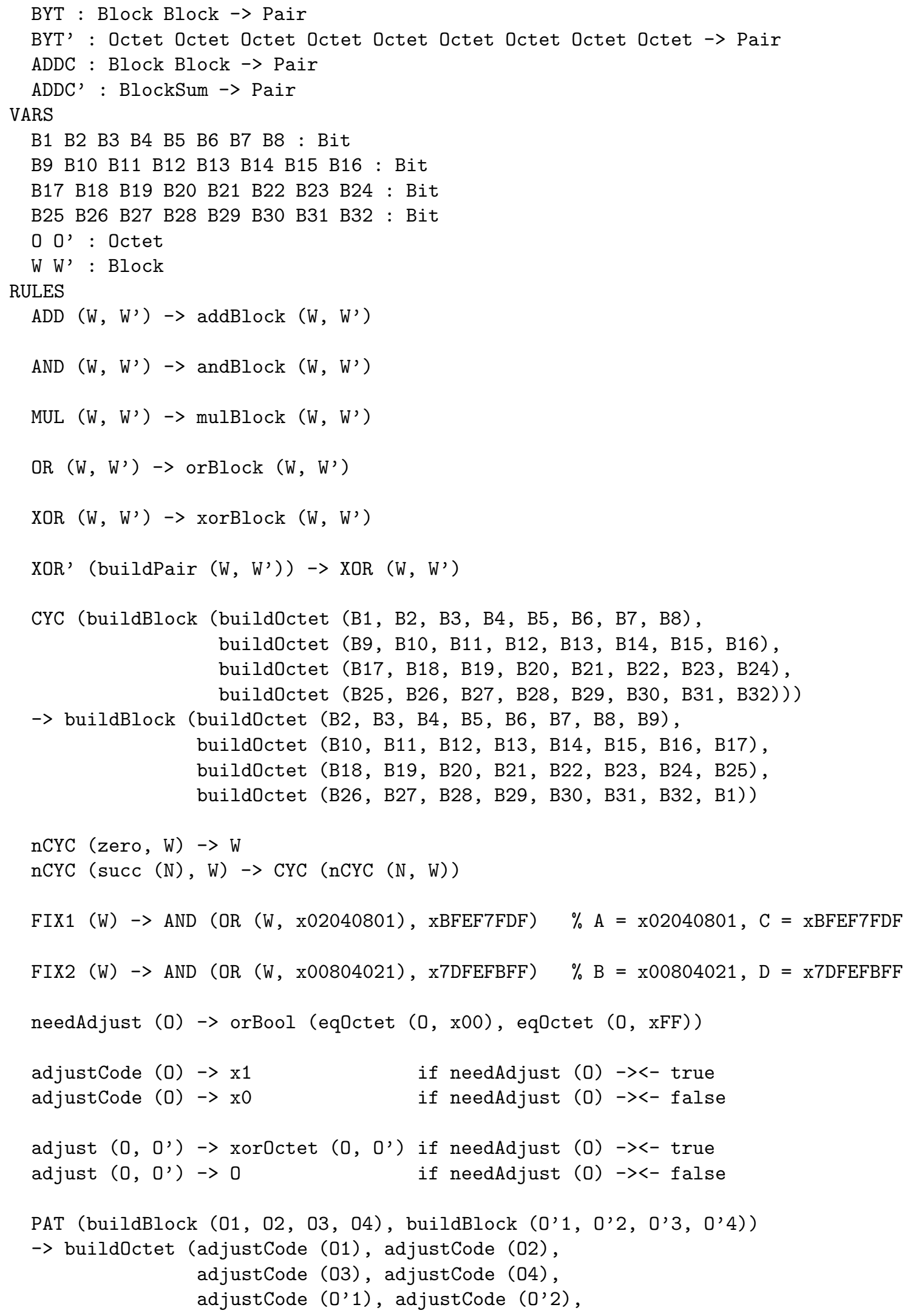


adjustCode (0’3), adjustCode (0’4))

BYT (buildBlock (01, 02, 03, 04), buildBlock (0'1, 0'2, 0'3, 0'4))

$\rightarrow$ BYT' (01, 02, 03, 04, 0’1, 0’2, 0’3, 0’4,

PAT (buildBlock (01, 02, 03, 04), buildBlock (0’1, 0’2, 0’3, 0’4)))

BYT' (01, 02, 03, 04, 0’1, 0’2, 0’3, 0’4, Opat)

-> buildPair (buildBlock (adjust (01, rightOctet7 (Opat)), adjust (02, right0ctet6 (Opat)), adjust (03, right0ctet5 (Opat)), adjust (04, right0ctet4 (Opat))), buildBlock (adjust (0’1, right0ctet3 (Opat)), adjust (0'2, right0ctet2 (Opat)), adjust (0'3, right0ctet1 (Opat)), adjust (0'4, Opat)))

$\operatorname{ADDC}\left(\mathrm{W}, \mathrm{W}^{\prime}\right) \rightarrow \operatorname{ADDC}\left(\operatorname{addBlockSum}\left(\mathrm{W}, \mathrm{W}^{\prime}\right)\right)$

ADDC' (buildBlockSum (x0, W)) $\rightarrow$ buildPair (x00000000, W)

ADDC' (buildBlockSum $(x 1, W)) \rightarrow$ buildPair (x00000001, W)

If needed, the four conditional rules could be eliminated by introducing two auxiliary functions adjustCode' and adjust' and modifying the definitions of adjustCode and adjust as follows:

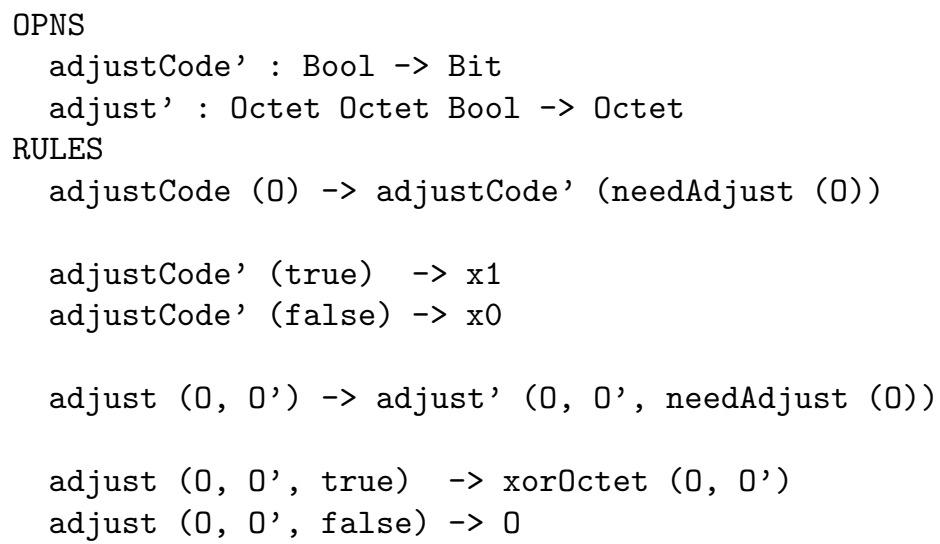

\section{B.15 Definitions (2) of MAA-specific cryptographic functions}

We now define a second set of functions, namely the "multiplicative" functions used for MAA computations. The three principal operations are MUL1, MUL2, and MUL2A; the other ones are auxiliary functions.

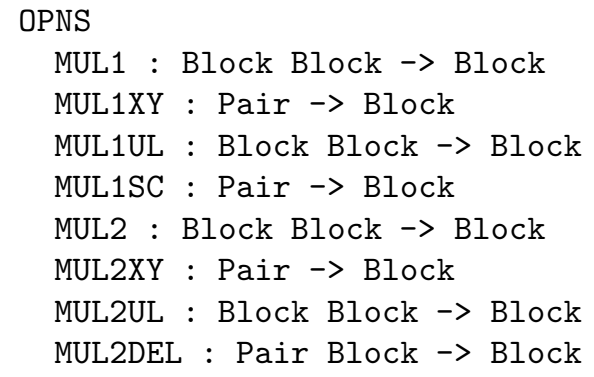




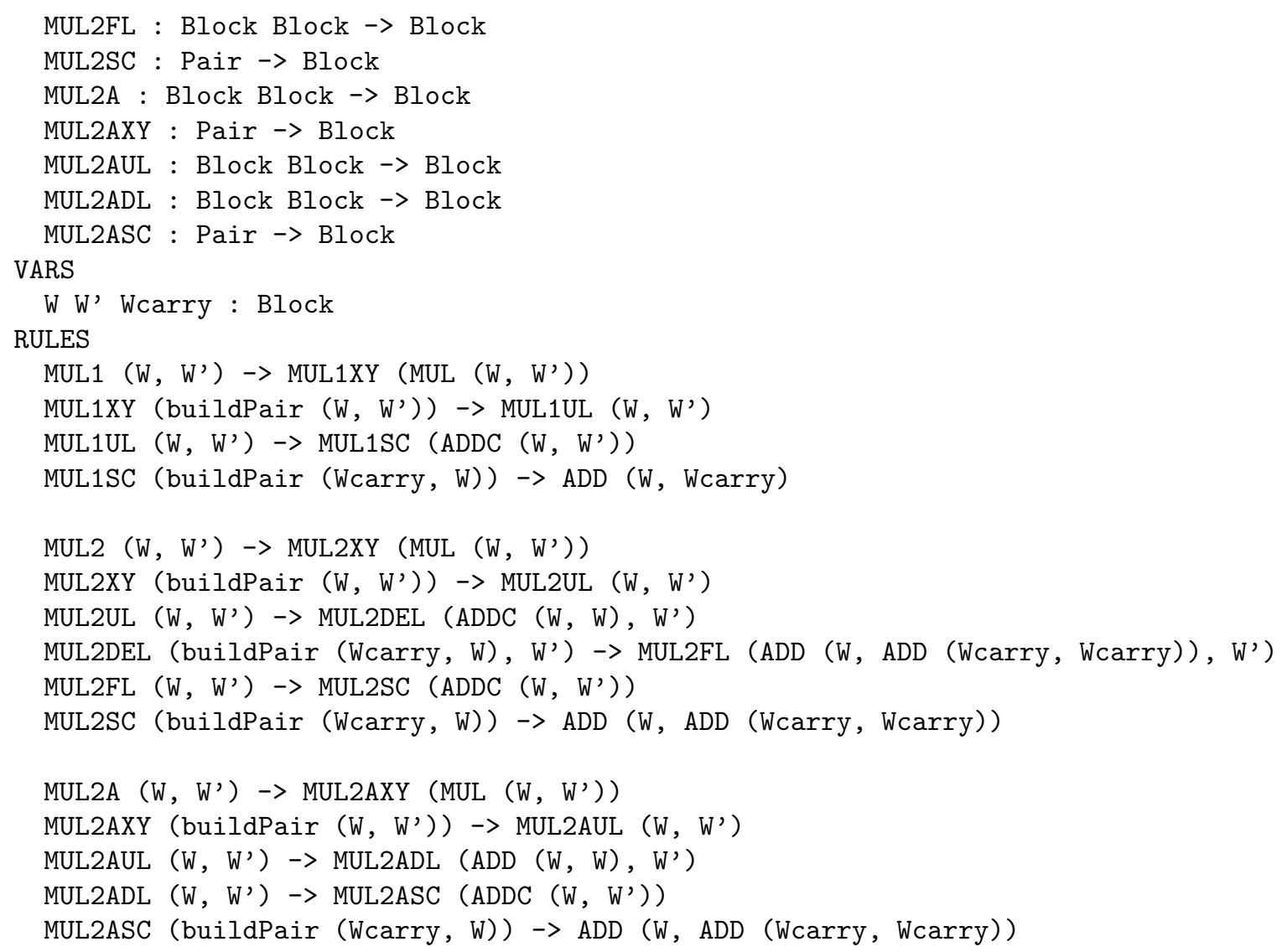

\section{B.16 Definitions (3) of MAA-specific cryptographic functions}

We now define a third set of functions used for MAA computations.

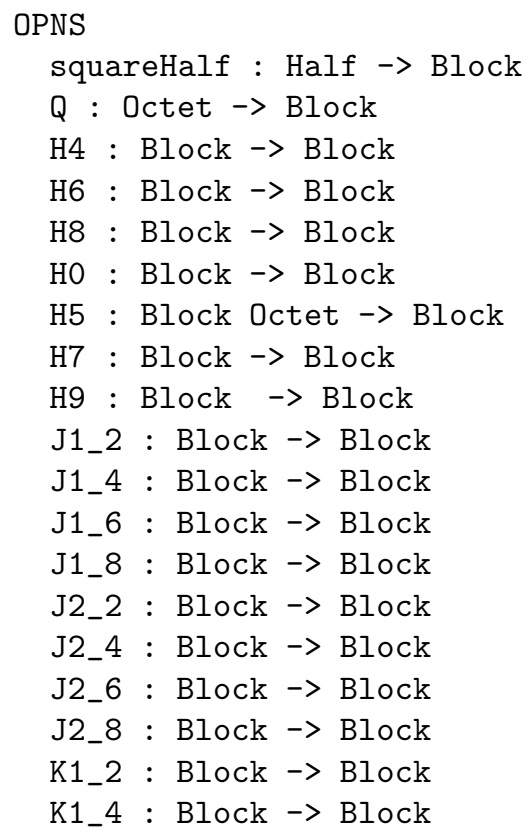




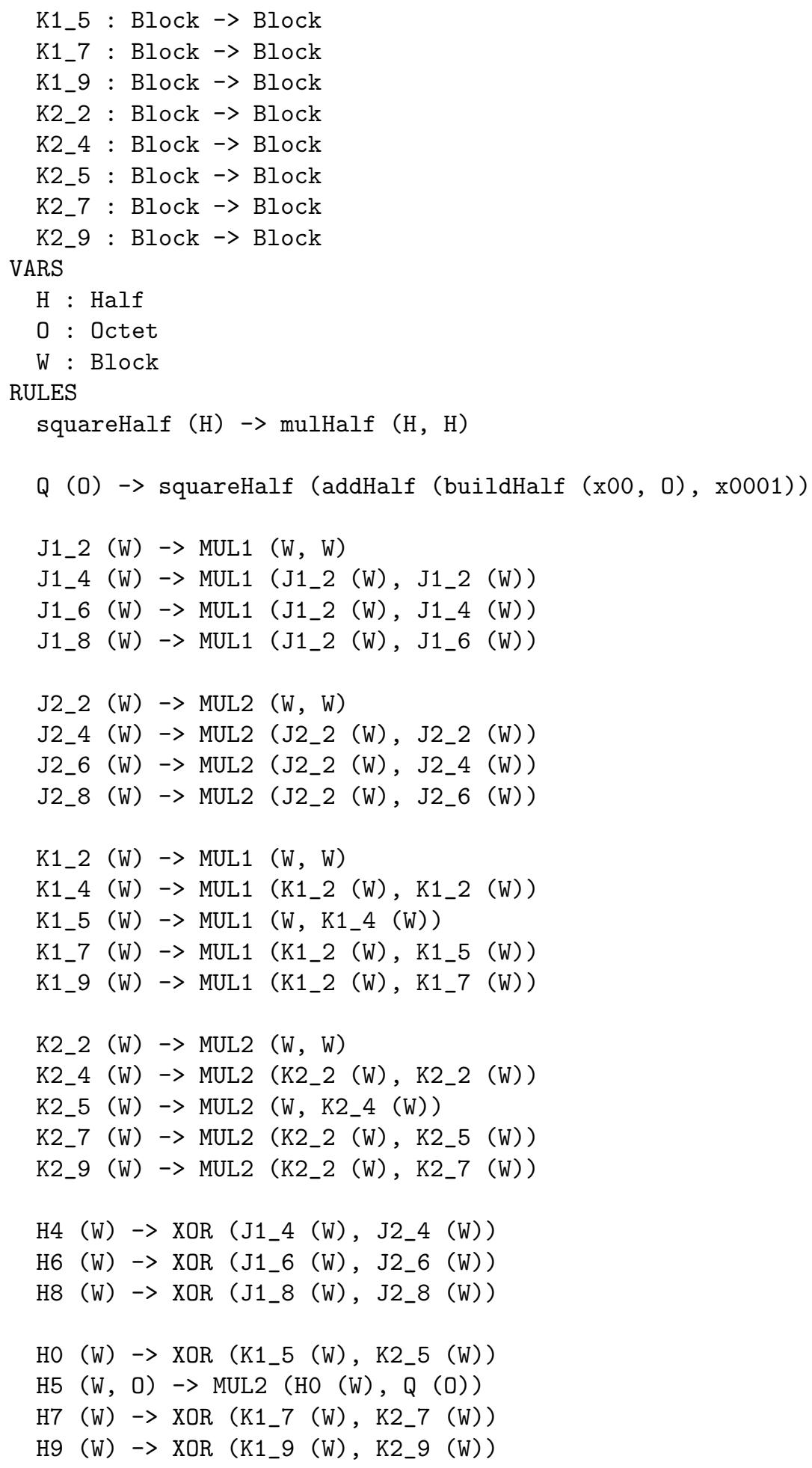




\section{B.17 Definitions (4) of MAA-specific cryptographic functions}

We now define the higher-level functions that implement the MAA algorithm, namely the prelude, the inner loop, and the coda; the two principal functions are MAA (which computes the signature of a nonsegmented message) and MAC (which splits a message into 1024-byte segments and computes the overall signature of this message by iterating on each segment, the 4-byte signature of each segment being prepended to the bytes of the next segment).

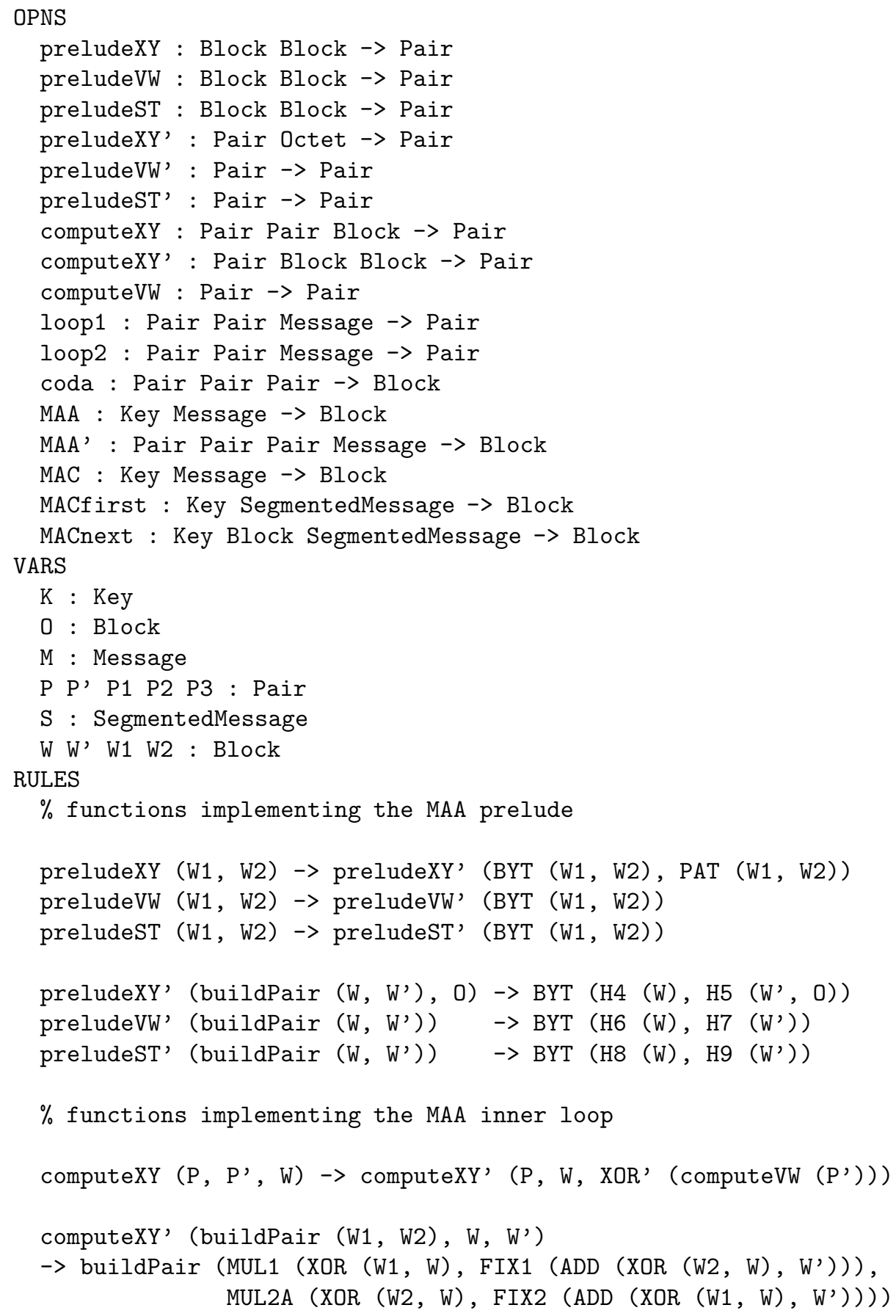




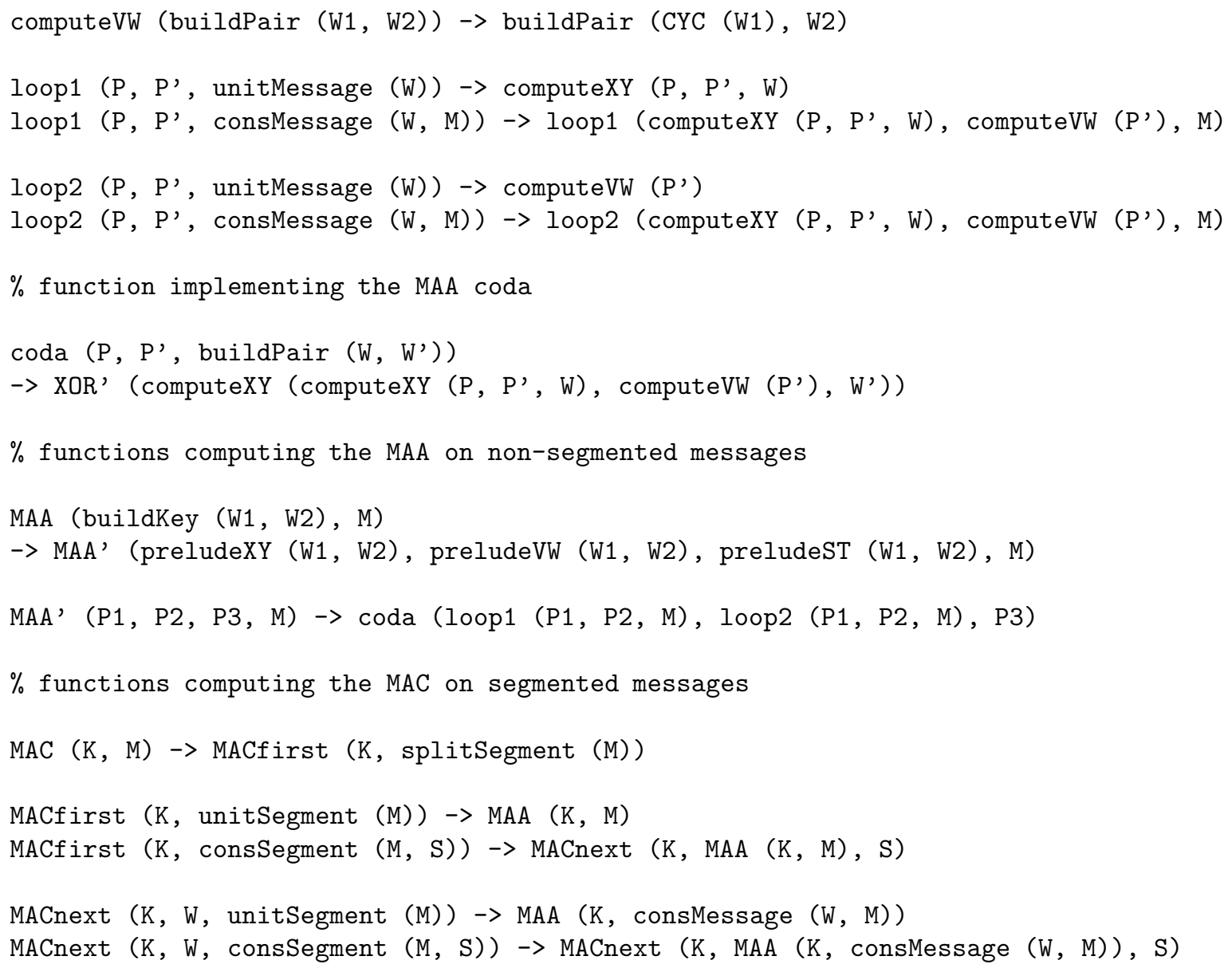

\section{B.18 Test vectors (1) for checking MAA computations}

We now define a first set of test vectors for the MAA. The following expressions implement the checks listed in Tables 1, 2, and 3 of [2] and should all evaluate to true if the MAA functions are correctly implemented.

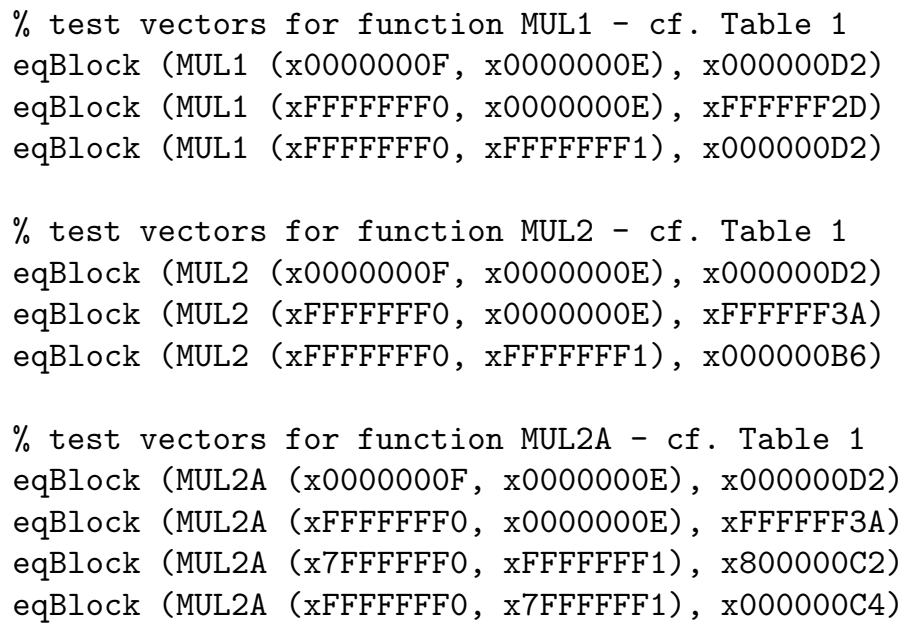




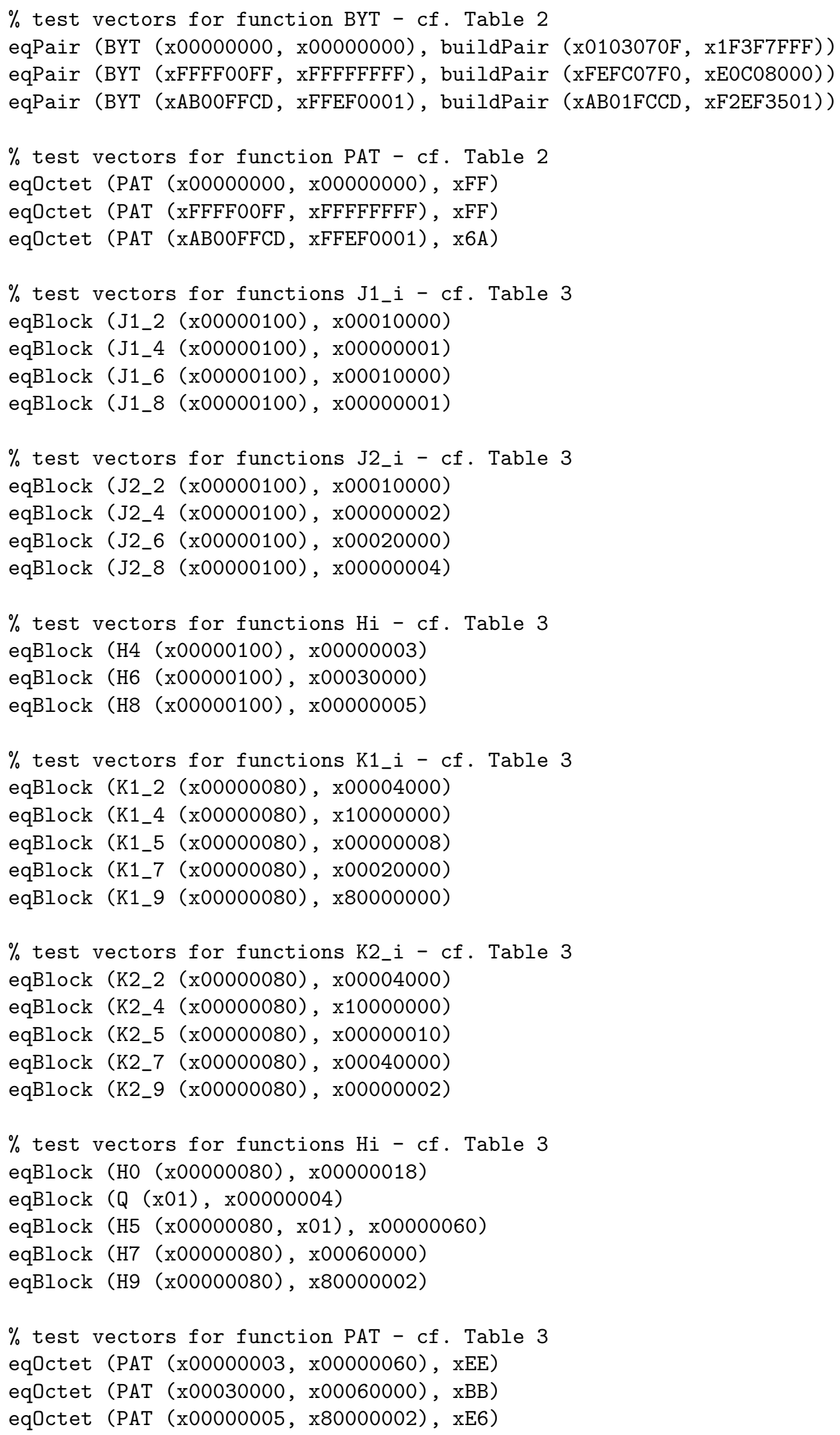


$\%$ test vectors for function BYT - inferred from Table 3

eqPair (BYT (x00000003, x00000060), buildPair (x01030703, x1D3B7760)) \% (X0, Y0)

eqPair (BYT (x00030000, x00060000), buildPair (x0103050B, x17065DBB)) \% (V0, W)

eqPair (BYT (x00000005, x80000002), buildPair $(\mathrm{x} 01030705, \mathrm{x} 80397302)) \%(\mathrm{~S}, \mathrm{~T})$

\section{B.19 Test vectors (2) for checking MAA computations}

We now define a second set of test vectors for the MAA, based upon Table 4 of [2]. The following expressions implement six groups of checks (three single-block messages and one three-block message). They should all evaluate to true if the main loop of MAA (as described page 10 of [2]) is correctly implemented.

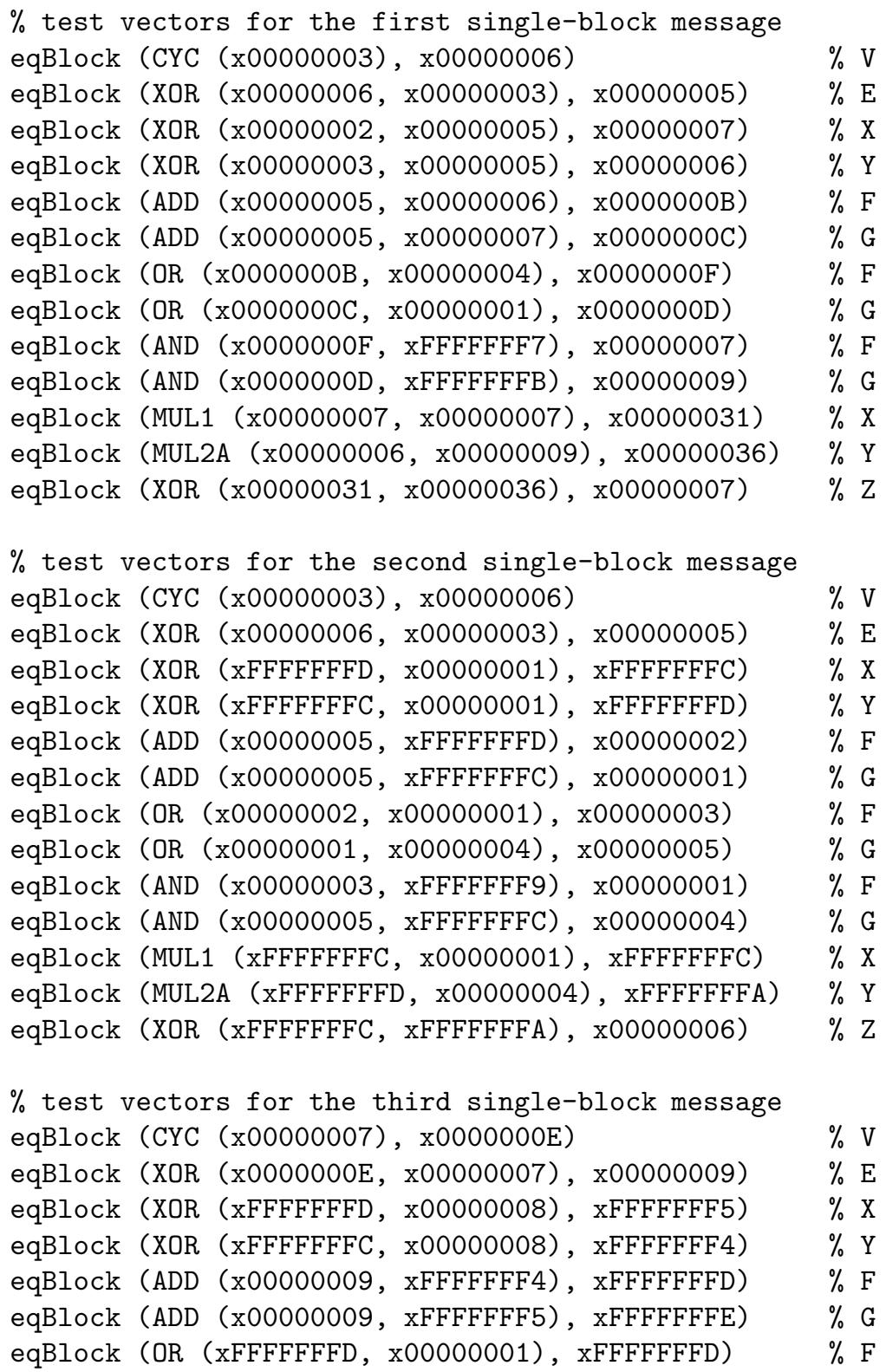




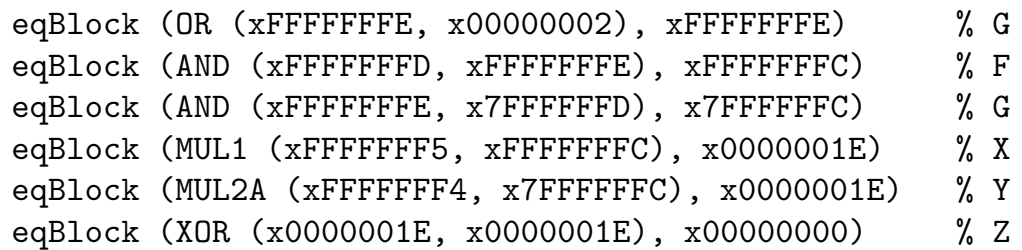

$\%$ test vectors for three-block message: first block eqBlock (CYC (x00000001), x00000002) \% V eqBlock (XOR (x00000002, x00000001), x00000003) \% E eqBlock (XOR (x00000001, x00000000), x00000001) \% X eqBlock (XOR (x00000002, x00000000), x00000002) \% eqBlock (ADD ( $x 00000003, x 00000002), x 00000005) \% F$ eqBlock (ADD (x00000003, x00000001), x00000004) \% G eqBlock (OR ( $x 00000005, x 00000002), x 00000007) \% F$ eqBlock (OR $(x 00000004, x 00000001), x 00000005) \quad \% G$ eqBlock (AND ( $x 00000007, x F F F F F F F B), x 00000003) \% F$ eqBlock (AND ( $x 00000005, x F F F F F F F B), x 00000001) \% G$ eqBlock (MUL1 ( $\mathrm{x} 00000001, \mathrm{x} 00000003), \mathrm{x} 00000003) \% \mathrm{X}$ eqB1ock (MUL2A (x00000002, x00000001), x00000002) \% Y eqBlock (XOR (x00000003, x00000002), x00000001) \% Z

$\%$ test vectors for the three-block message: second block eqBlock (CYC (x00000002), x00000004) \% V eqBlock (XOR (x00000004, x00000001), x00000005) \% E eqBlock (XOR ( $x 00000003, x 00000001), x 00000002) \% x$ eqBlock (XOR ( $x 00000002, x 00000001), x 00000003) \% y$ eqBlock (ADD ( $x 00000005, x 00000003), x 00000008) \% F$ eqBlock (ADD $(x 00000005, x 00000002), x 00000007) \% G$ eqBlock (OR (x00000008, x00000002), x0000000A) \% F eqBlock (OR (x00000007, x00000001), x00000007) \% G eqBlock (AND ( $\mathrm{x} 0000000 \mathrm{~A}, \mathrm{xFFFFFFFB}), \mathrm{x} 0000000 \mathrm{~A}) \% \mathrm{~F}$ eqBlock (AND ( $\mathrm{x} 00000007, \mathrm{xFFFFFFFB)}, \mathrm{x} 00000003) \% \mathrm{G}$ eqBlock (MUL1 ( $x 00000002, x 0000000 A), x 00000014) \% x$ eqBlock (MUL2A ( $\mathrm{x} 00000003, \mathrm{x} 00000003), \mathrm{x} 00000009) \% \mathrm{Y}$ eqBlock (XOR (x00000014, x00000009), x0000001D) \% Z

$\%$ test vectors for three-block message: third block eqBlock (CYC (x00000004), x00000008) \% V eqBlock (XOR (x00000008, x00000001), x00000009) \% E eqBlock (XOR (x00000014, x00000002), x00000016) \% X eqBlock (XOR (x00000009, x00000002), x0000000B) \% Y eqBlock (ADD (x00000009, x0000000B), x00000014) \% F eqBlock (ADD $(x 00000009, x 00000016), x 0000001 F) \%$ G eqBlock (OR ( $x 00000014, x 00000002), x 00000016) \% F$ eqBlock (OR (x0000001F, x00000001), x0000001F) \% G eqBlock (AND ( $x 00000016, x F F F F F F F B), x 00000012) \% F$ eqBlock (AND ( $x 0000001 F, x F F F F F F F B), x 0000001 B$ ) \% G eqBlock (MUL1 ( $x 00000016, x 00000012), x 0000018 C) \% x$ eqBlock (MUL2A (x0000000B, x0000001B), x00000129) \% Y eqBlock (XOR (x0000018C, x00000129), x000000A5) \% Z 
We complete the above tests with additional test vectors taken from [10, Annex E.3.3], which only gives detailed values for the first block of the 84-block test message.

\% test vectors for block x0A202020 with key $(\mathrm{J}=\mathrm{xE6A12F07,} \mathrm{K}=\mathrm{x} 9 \mathrm{D} 15 \mathrm{C} 437$ )
eqBlock (CYC (xC4EB1AEB), x89D635D7)
eqBlock (XOR (x89D635D7, xF6A09667), x7F76A3B0)
eqBlock (XOR (x21D869BA, x0A202020), x2BF8499A)
eqBlock (XOR (x7792F9D4, x0A202020), x7DB2D9F4)
eqBlock (ADD (x7F76A3B0, x7DB2D9F4), xFD297DA4)
eq $\%$ F
eqBlock (ADD (x7F76A3B0, x2BF8499A), xAB6EED4A)
eqBlock (OR (xFD297DA4, x02040801), xFF2D7DA5)
eqBlock (OR (xAB6EED4A, x00804021), xABEEED6B)
eqBlock (AND (xFF2D7DA5, xBFEF7FDF), xBF2D7D85)
eqBlock (AND (xABEEED6B, x7DFEFBFF), x29EEE96B)
eqBlock (MUL1 (x2BF8499A, xBF2D7D85), x0AD67E20)
eqBlock (MUL2A (x7DB2D9F4, x29EEE96B), x30261492)

\section{B.20 Test vectors (3) for checking MAA computations}

We now define a third set of test vectors for the MAA, based upon Table 5 of [2]. The following expressions implement four groups of checks, with two different keys and two different messages. They should all evaluate to true if the MAA signature is correctly computed.

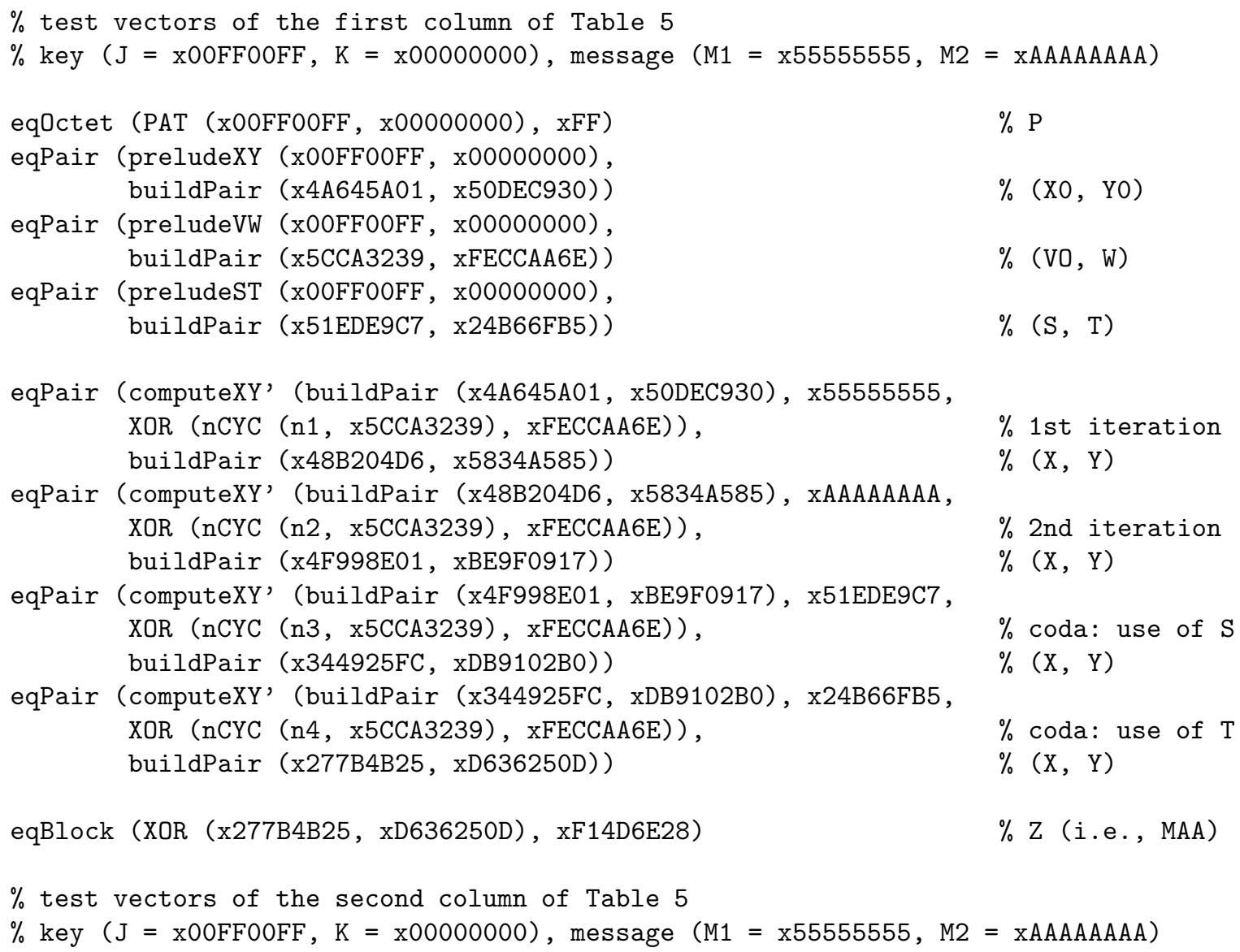




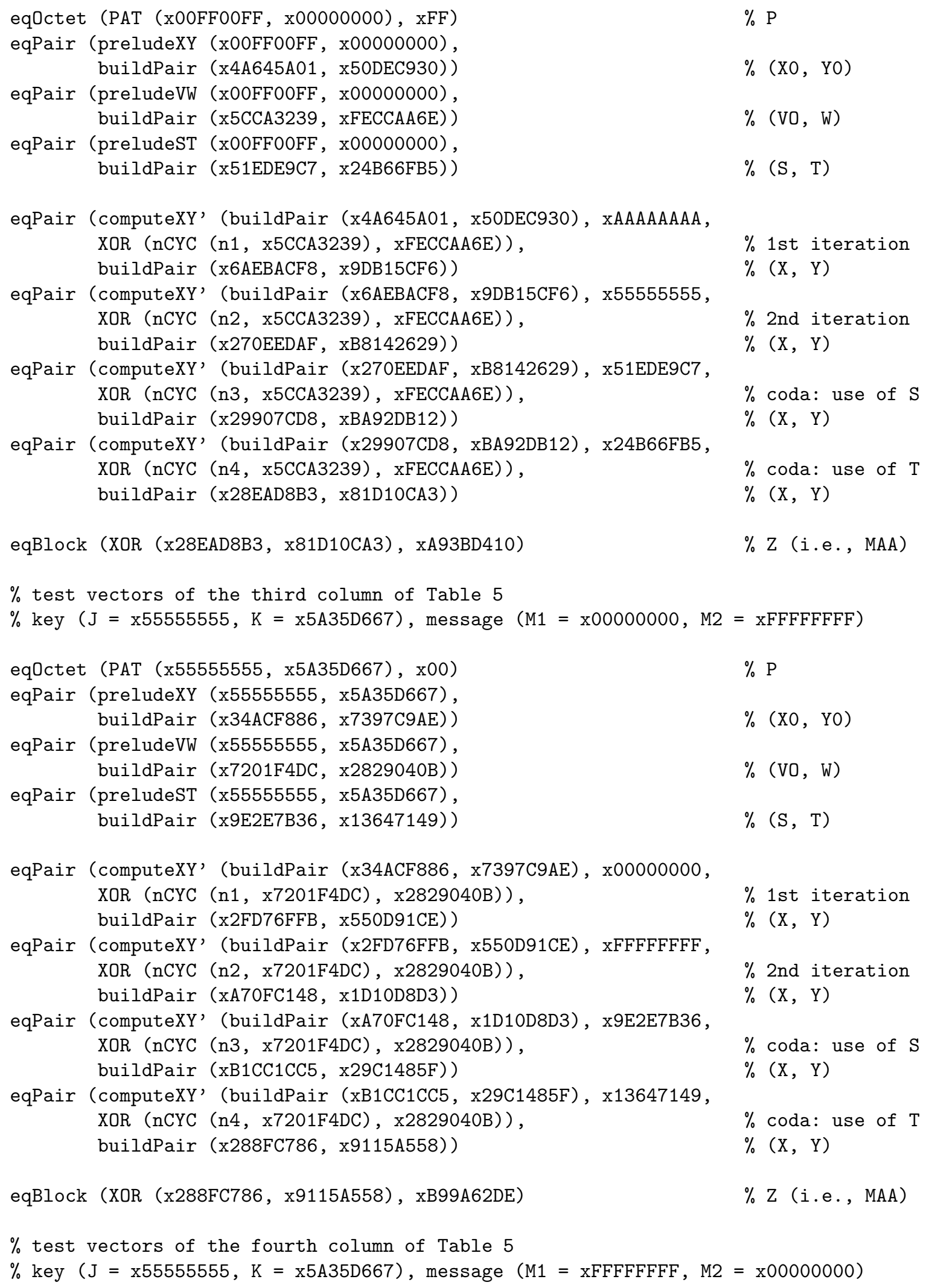




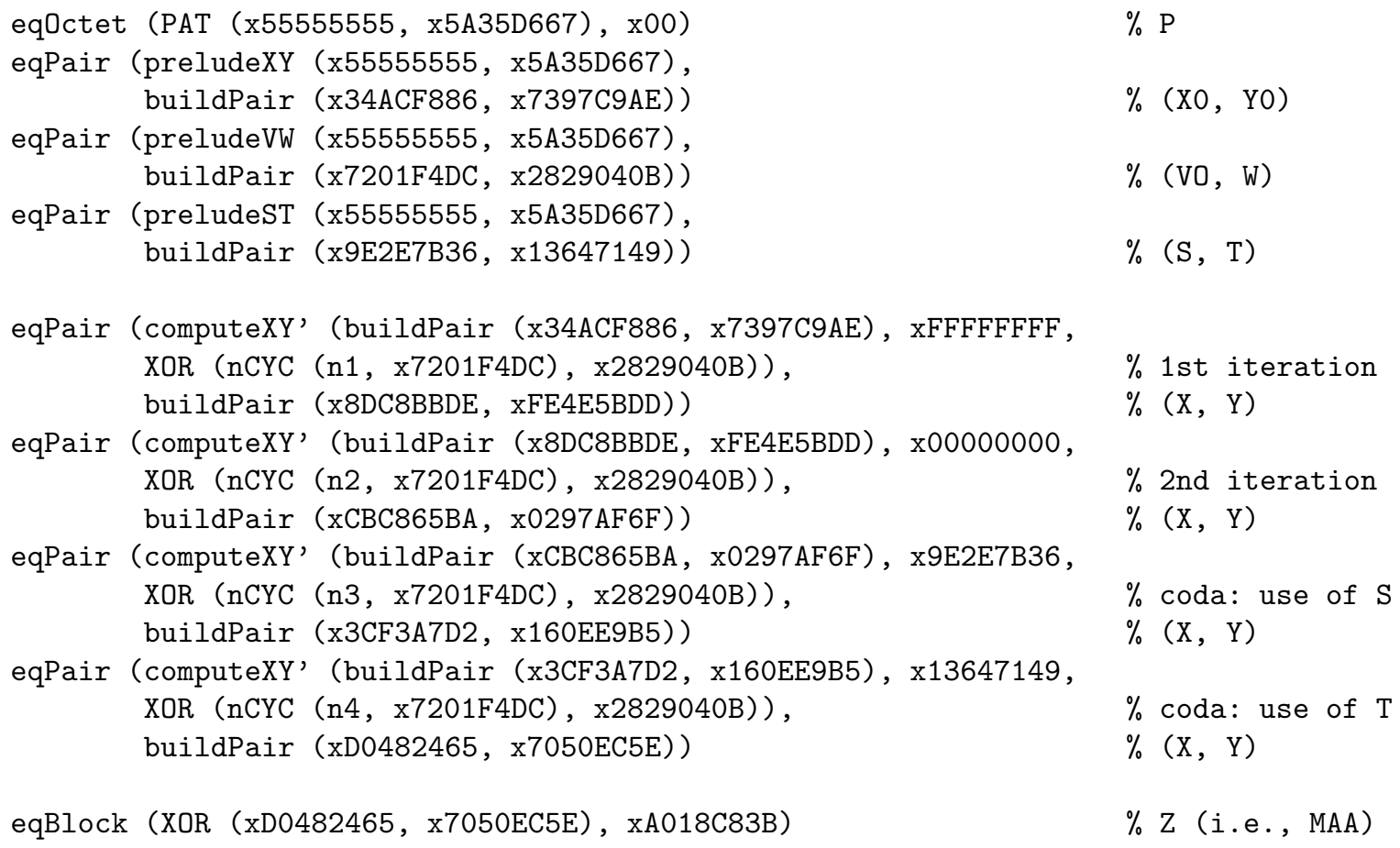

We complete the above tests with additional test vectors taken from [10, Annex E.3.3], which gives prelude results computed for another key.

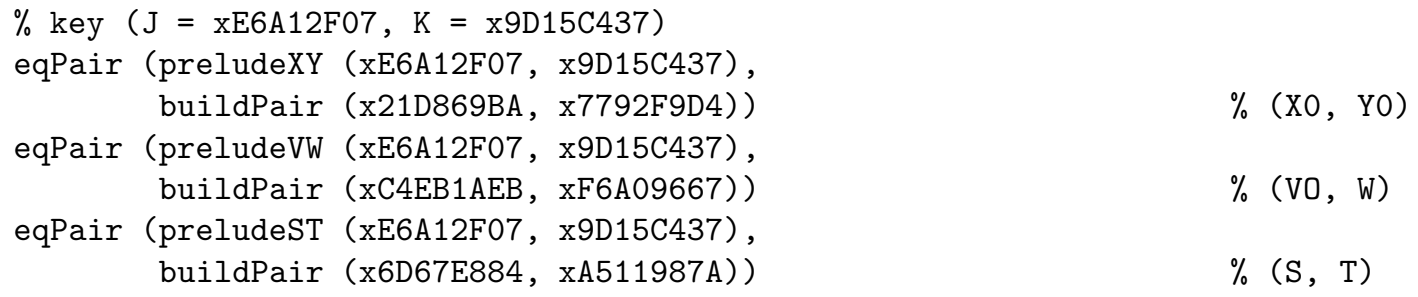

\section{B.21 Test vectors (4) for checking MAA computations}

We define a last set of test vectors for the MAA. The first one (a message of 20 blocks containing only zeros) was directly taken from Table 6 of [2].

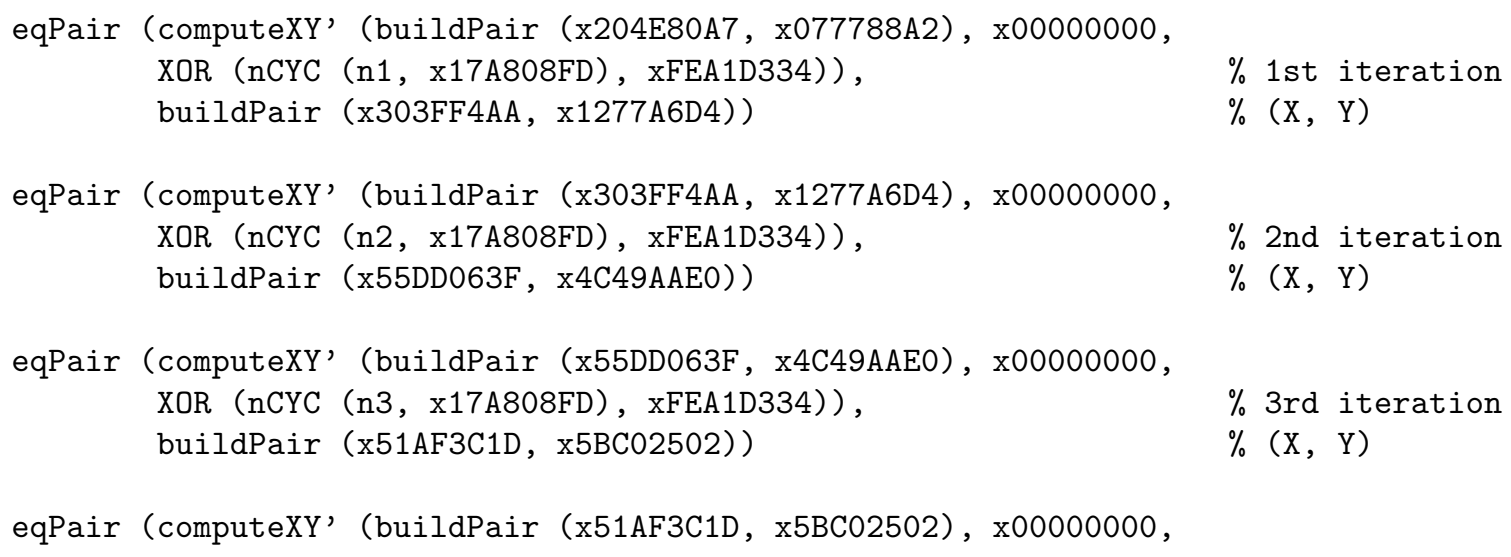




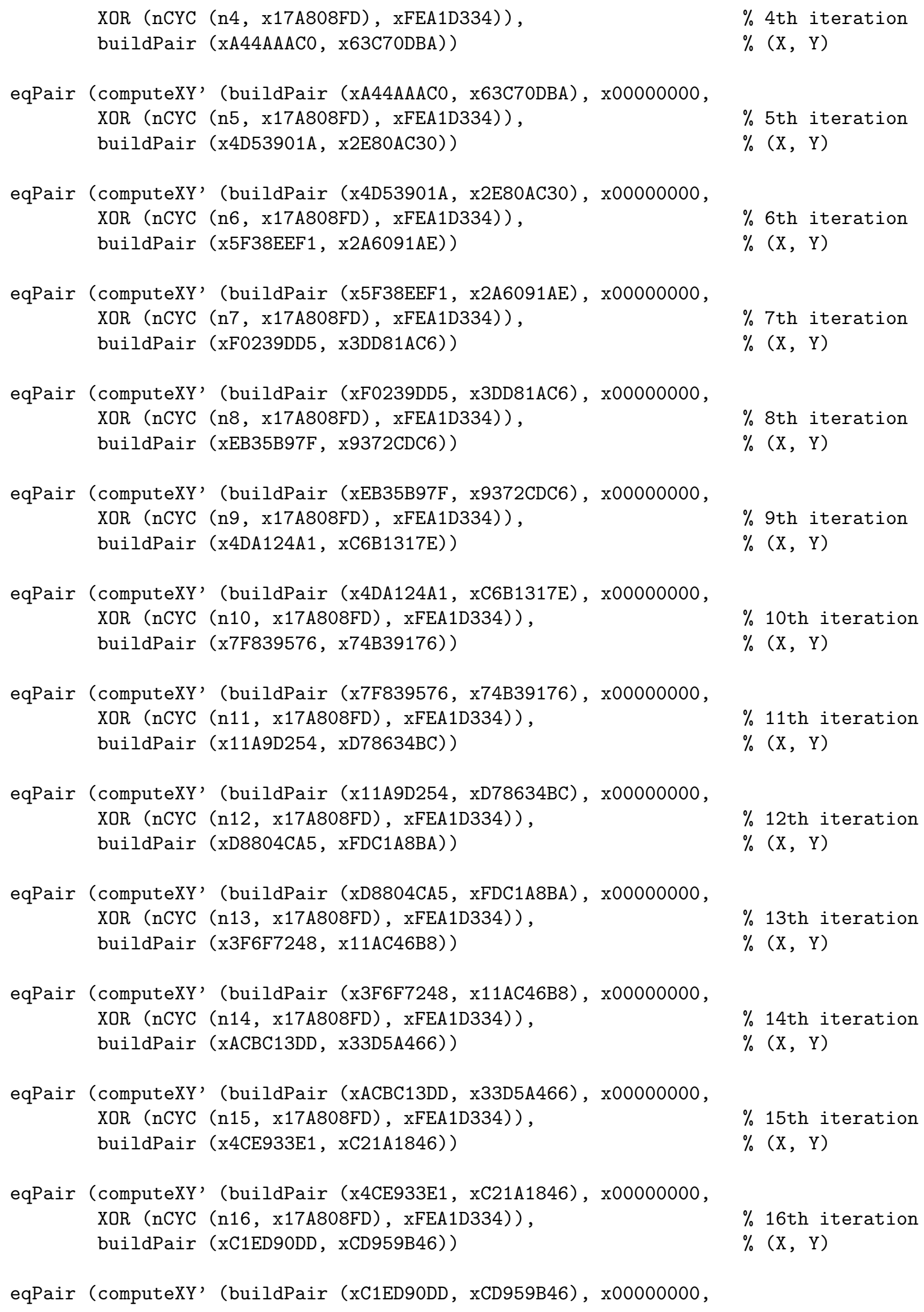

eqPair (computeXY' (buildPair (xC1ED90DD, xCD959B46), x00000000, 


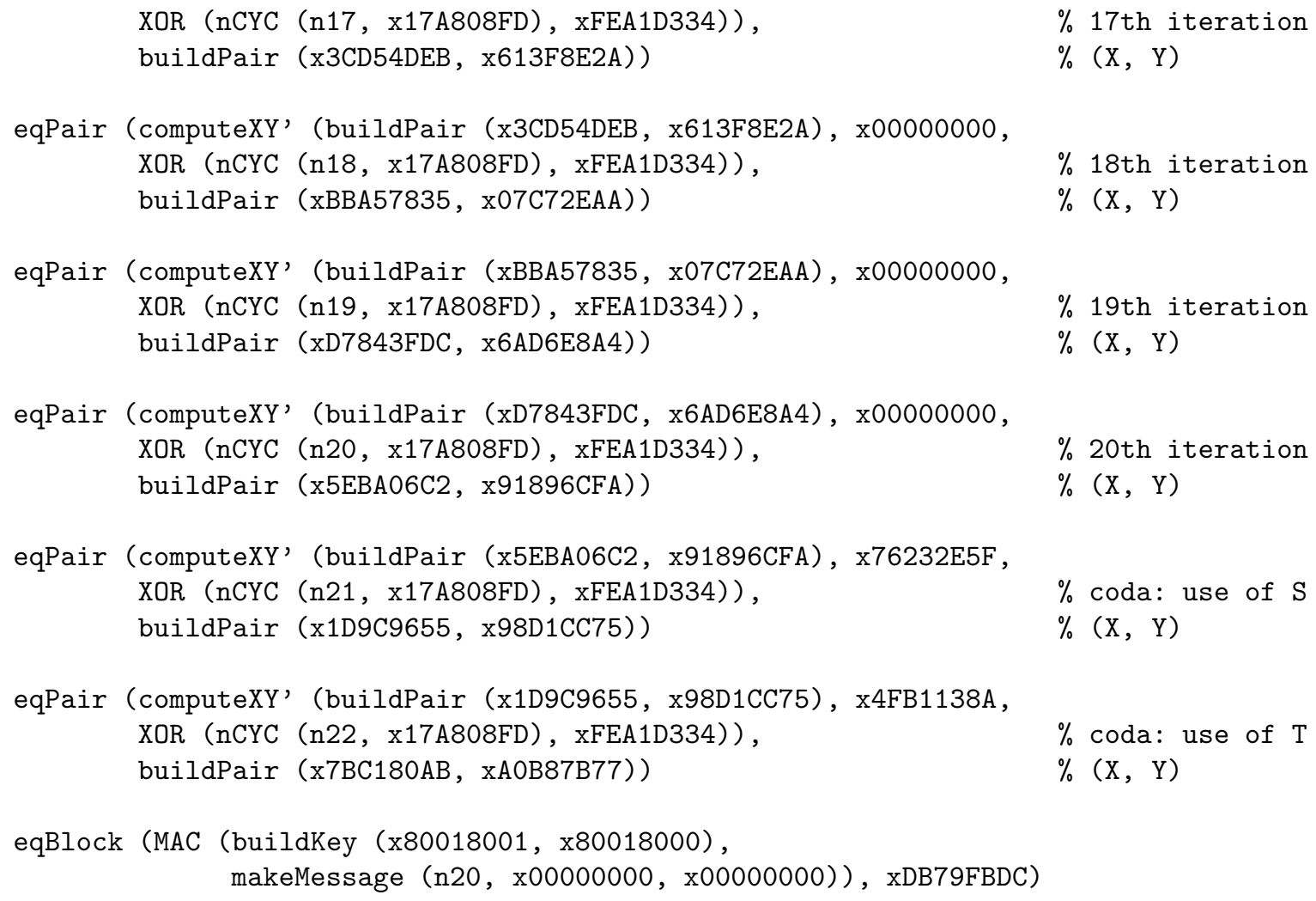

We believe that the test vector above is not sufficient to detect implementation mistakes arising from byte permutations (e.g., endianness issues) or incorrect segmentation of messages longer than 1024 bytes (i.e., 256 blocks). To address these issues, we added three supplementary test vectors that operate on messages of 16, 256, and 4100 blocks containing bit patterns not preserved by permutations.

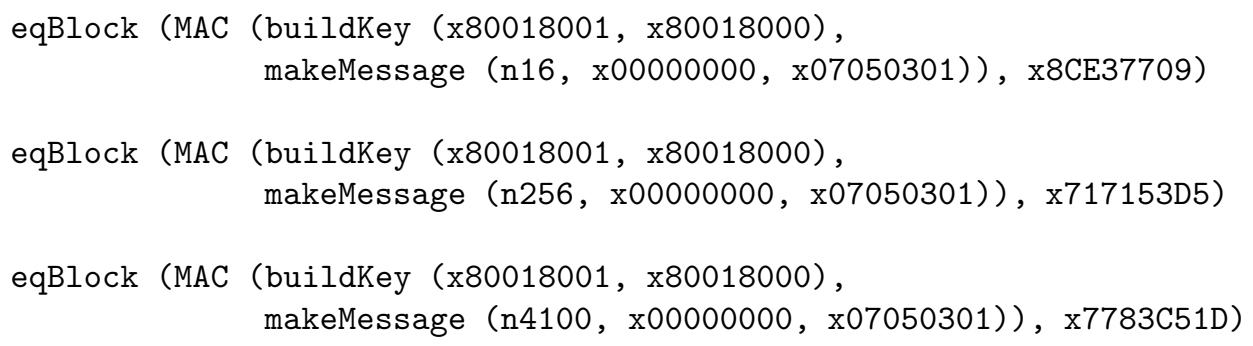

\section{B.22 Possible variants}

The REC specification given in the present Annex could be enhanced in two directions that diverge from the modelling choices originally done in [15] and could be given as exercises to students:

- At present, the Prelude function is called several times when computing the MAC value for a given message; precisely, this function is called for every 256-block segment of the message. This is neither useful nor efficient. Propose a modification of the REC specification to ensure that the Prelude function is called only once per message.

- Before computing the MAC value for a given message, the REC specification converts this message into a segmented message by calling the splitSegment function. Actually, such a prelim- 
inary duplication of message contents is not mandatory and could be avoided. Propose a modification of the REC specification in which the SegmentedMessage sort and all the definitions of Section B.13 are removed, so that the MAC value is directly computed using a one-pass traversal of the message list, from its head to its tail, still taking the MAA "mode of operation" into account. 